\title{
Ising Model: Local Spin Correlations and Conformal Invariance
}

\author{
Reza Gheissari ${ }^{1}$, Clément Hongler ${ }^{2}$, S. C. Park ${ }^{2}$ \\ ${ }^{1}$ Courant Institute of Mathematical Sciences, New York University, 251 Mercer St., New York, NY 10012, \\ USA. E-mail: reza@cims.nyu.edu \\ 2 Ecole Polytechnique Fédérale de Lausanne, EPFL SB MATHAA CSFT, 1015 Lausanne, Switzerland. \\ E-mail: clement.hongler@epfl.ch; sungchul.park@epfl.ch
}

Received: 1 December 2016 / Accepted: 16 November 2018

Published online: 14 February 2019 - (C) The Author(s) 2019, Corrected Publication March/2019

\begin{abstract}
We study the 2-dimensional Ising model at critical temperature on a simply connected subset $\Omega_{\delta}$ of the square grid $\delta \mathbb{Z}^{2}$. The scaling limit of the critical Ising model is conjectured to be described by Conformal Field Theory; in particular, there is expected to be a precise correspondence between local lattice fields of the Ising model and the local fields of Conformal Field Theory. Towards the proof of this correspondence, we analyze arbitrary spin pattern probabilities (probabilities of finite spin configurations occurring at the origin), explicitly obtain their infinite-volume limits, and prove their conformal covariance at the first (non-trivial) order. We formulate these probabilities in terms of discrete fermionic observables, enabling the study of their scaling limits. This generalizes results of Hongler (Conformal invariance of Ising model correlations. Ph.D. thesis, [Hon10]), Hongler and Smirnov (Acta Math 211(2):191-225, [HoSm13]), Chelkak, Hongler, and Izyurov (Ann. Math. 181(3), 1087-1138, [CHI15]) to one-point functions of any local spin correlations. We introduce a collection of tools which allow one to exactly and explicitly translate any spin pattern probability (and hence any lattice local field correlation) in terms of discrete complex analysis quantities. The proof requires working with multipoint lattice spinors with monodromy (including construction of explicit formulae in the full plane), and refined analysis near their source points to prove convergence to the appropriate continuous conformally covariant functions.
\end{abstract}

\section{Contents}

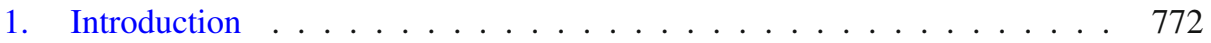

2. Discrete Complex Analysis . . . . . . . . . . . . . . . . . . 783

3. Discrete Two-Point Observables . . . . . . . . . . . . . . . . . . 786

4. Discrete Multipoint Observables . . . . . . . . . . . . . . . . . . . . 799

5. Scaling Limits of Observables . . . . . . . . . . . . . . . . . . . . 813

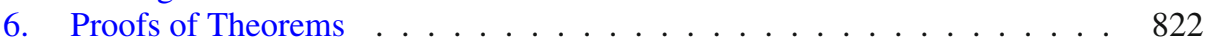

A. The Harmonic Measure on $\mathbb{C}_{1} \backslash \mathbb{R}_{>0} \ldots \ldots \ldots \ldots$ 
B. Contour Weights . . . . . . . . . . . . . . . . . . . . 828

C. Explicit Pattern Probabilities . . . . . . . . . . . . . . . . 830

\section{Introduction}

The 2D Ising model is one of the most studied models of statistical mechanics. In its simplest formulation it consists of a random assignment of \pm 1 spins $\sigma_{x}$ to the faces of (subgraphs of) the square grid $\mathbb{Z}^{2}$ (Fig. 1); the spins tend to align with their neighbors; the probability of a configuration is proportional to $e^{-\beta H(\sigma)}$ where the energy $H(\sigma)=$ $-\sum_{i \sim j} \sigma_{i} \sigma_{j}$ sums over pairs of adjacent faces; alignment strength is controlled by the parameter $\beta>0$, usually identified with the inverse temperature.

The 2D Ising model has found applications in many areas of science, from description of magnets to ecology and image processing. Due to its simplicity and emergent features, it is interesting both as a discrete probability and statistical field theory model. Of particular physical interest is the phase transition at the critical point $\beta_{c}$ : for $\beta<\beta_{c}$ the system is disordered at large scales while for $\beta>\beta_{c}$ a long-range ferromagnetic order arises. Classically, the phase transition can be described in terms of the infinitevolume limit: in the disordered phase $\beta<\beta_{c}$ there is a unique Gibbs measure, while in the ordered phase $\beta>\beta_{c}$ infinite-volume measures are convex combinations of two extremal measures. It has a continuous phase transition: only one Gibbs measure exists at $\beta=\beta_{c}$.

Critical lattice models at continuous phase transition points are widely expected to have universal scaling limits (independent of the choice of lattice and other details). In 2D, such scaling limits are expected to exhibit conformal symmetry. This can be loosely formulated as follows: for a conformal mapping $\varphi: \Omega \rightarrow \tilde{\Omega}$,

$$
\varphi(\text { scaling limit on } \Omega)=\text { scaling limit on } \tilde{\Omega} \text {. }
$$

There are two main tools used to describe the scaling limits of planar lattice models: curves and fields. Schramm-Loewner Evolution (SLE) curves naturally arise in conformally invariant setups: for the Ising model, they describe the scaling limit of interfaces between opposite spins [CDHKS14,BeHo16]. The fields on a discrete level, such as the \pm 1 -valued spin field $\sigma_{i}$, can be described by Conformal Field Theory (CFT): their correlations, in principle, are conjecturally described using representationtheoretic methods. Such conjectures have been proved for a number of natural fields [Hon10,HoSm13,CHI15]; the present paper is part of this program.

What makes it possible to mathematically analyze the 2D Ising model with great precision is its exactly solvable structure, first revealed by Onsager [Ons44]. The exact solvability can be formulated in many different ways; in recent years, the formulation in terms of discrete complex analysis has emerged as one the most powerful ways to understand the scaling limit of the model rigorously. In particular, the model's conformal symmetry becomes much more transparent in this context.

The results of [Hon10] and [CHI15] on (asymptotic) conformal invariance of spin and energy fields can be formulated, in their simplest cases, as follows: consider the critical Ising model with plus boundary conditions on the discretization $\Omega_{\delta}$ by a square grid of mesh size $\delta>0$ of a simply-connected domain $\Omega$ around the origin. Take the origin $0 \in \Omega$, identify it with the closest face of $\Omega_{\delta}$ and let $\delta$ be the face to the right of 0 . Then, as $\delta \rightarrow 0$, we have the asymptotic expansions, 


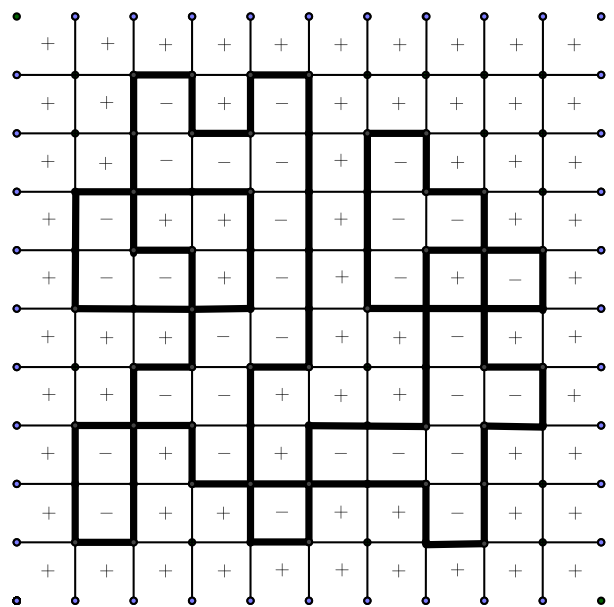

Fig. 1. An Ising model configuration on the faces of a square subset of $\mathbb{Z}^{2}$ with all-plus boundary conditions, along with its "low-temperature expansion", indicating interfaces separating plus and minus spins

[spin field]

$$
\mathbb{E}_{\Omega_{\delta}}\left[\sigma_{0}\right]=0+C_{\sigma}\left|\varphi^{\prime}(0)\right|^{-\frac{1}{8}} \delta^{\frac{1}{8}}+o\left(\delta^{\frac{1}{8}}\right)
$$

[energy density field]

$$
\mathbb{E}_{\Omega_{\delta}}\left[\sigma_{0} \sigma_{\delta}\right]=\frac{\sqrt{2}}{2}+C_{\epsilon}\left|\varphi^{\prime}(0)\right|^{-1} \delta+o(\delta),
$$

where $C_{\sigma}, C_{\epsilon}>0$ are explicit (lattice-dependent) constants and $\varphi$ is any conformal map from the unit disk $\mathbb{D}$ to $\Omega$ fixing the origin. The first terms in the respective expansions are the infinite-volume limits of the left-hand side quantities. These results illustrate the following: for any local field one-point function, the correction to its infinite-volume expectation is described by Conformal Field Theory (CFT) quantities.

The Ising Model is conjectured to be described by the unitary CFT minimal model $\mathcal{M}_{3,4}$ (see e.g. [BPZ84], [DMS97]), also known as the Ising CFT. The Ising CFT consists of three primary fields: those of respective scaling dimensions 0 (the identity field a constant field identically equal to one), $\frac{1}{8}$ (the spin field) and 1 (the energy field). Each of these primary fields generates an infinite-dimensional tower of fields called its descendants.

We conjecture that the space of the Ising CFT fields describes the limits of Ising lattice local fields:

Definition. Let $\mathcal{F}$ be a finite connected collection of faces of $\mathbb{Z}^{2}$ including 0 . For any $F:\{ \pm 1\}^{\mathcal{F}} \rightarrow \mathbb{C}$, a lattice local field $\Phi_{\delta}^{F}$ is a random field on the faces of $\Omega_{\delta}$ whose values are given by $\Phi_{\delta}^{F}(x)=F\left(\left.\sigma\right|_{x+\delta \mathcal{F}}\right)$. We call a local field spin-antisymmetric if $F(-\sigma)=-F(\sigma)$ and spin-symmetric if $F(-\sigma)=F(\sigma)$.

Conjecture. For any nonzero lattice field (i.e. whose correlations do not vanish generically; see $[\mathrm{HKV} 17]) \Phi_{\delta}^{F}$, there exists $D \in \mathbb{N} \cup\left(\mathbb{N}+\frac{1}{8}\right)$ such that

$$
\delta^{-D} \Phi_{\delta}^{F} \rightarrow \Phi
$$

in the sense of correlations (meaning that the $n$ point functions converge), where $\Phi$ is a nonzero primary or descendant CFT field. If $\Phi$ is spin-antisymmetric then $D \in \mathbb{N}+\frac{1}{8}$; if $\Phi$ is spin-symmetric then $D \in \mathbb{N}$. Moreover every Ising CFT field can be obtained in such a manner. 
Spin pattern probabilities Correlations of any lattice local field at a point $x$ can be rewritten in terms of probabilities of observing certain spin patterns centered at $x$, i.e. probabilities of spin configurations in a microscopic neighborhood of $x$. The main objective of this paper is to obtain explicit representations for probabilities of spin pattern events, which are the most general local quantities describing the model: we obtain infinite-volume limits for arbitrary local pattern probabilities and give their first-order corrections corresponding to what can be expected from the Ising-CFT correspondence (see Theorems 1.1-1.2 and Corollary 1.3).

More precisely, Let $\mathcal{F}$ be a finite connected set of faces in $\mathbb{Z}^{2}$ and fix a configuration $\rho \in\{ \pm 1\}^{\mathcal{F}}$. We look at two types of lattice local fields:

- spin-antisymmetric pattern fields $\Phi_{\delta}^{F_{\rho}}(x)$ where $F_{\rho}=\mathbf{1}\left\{\left.\sigma\right|_{\mathcal{F}}=\rho\right\}$, whose expectation gives the probability of the spin-antisymmetric pattern $\rho$ on $\mathcal{F}$,

- spin-symmetric pattern fields $\Phi_{\delta}^{F_{\rho}^{ \pm}}(x)$ where $F_{\rho}^{ \pm}=\mathbf{1}\left\{\left.\sigma\right|_{\mathcal{F}} \in\{ \pm \rho\}\right\}$, whose expectation gives the probability of the spin-symmetric pattern $\pm \rho$ on $\mathcal{F}$.

Every Ising lattice local field can easily be seen to be a finite linear combination of such fields. The main result of the paper is the following.

Theorem (see Corollary 1.3). Let $\mathcal{F}$ and $\rho$ be as above and let $\mathbb{P}_{\mathbb{Z}^{2}}$ be the infinite-volume measure of the critical Ising model. Consider the critical Ising model on $\Omega_{\delta}$ with plus boundary conditions, and denote it by $\mathbb{P}_{\Omega_{\delta}}$. Then as $\delta \rightarrow 0$, we have

$$
\begin{aligned}
\mathbb{P}_{\Omega_{\delta}}\left[\left.\sigma\right|_{\delta \mathcal{F}}=\rho\right] & =\mathbb{P}_{\mathbb{Z}^{2}}\left[\left.\sigma\right|_{\mathcal{F}}=\rho\right]+\delta^{\frac{1}{8}} \cdot \text { geometric effect }(\rho, \Omega)+o\left(\delta^{\frac{1}{8}}\right), \\
\mathbb{P}_{\Omega_{\delta}}\left[\left.\sigma\right|_{\delta \mathcal{F}} \in\{ \pm \rho\}\right] & =\mathbb{P}_{\mathbb{Z}^{2}}\left[\left.\sigma\right|_{\mathcal{F}} \in\{ \pm \rho\}\right]+\delta \cdot \text { geometric effect }( \pm \rho, \Omega)+o(\delta) .
\end{aligned}
$$

The infinite-volume probability and the geometric effects are given in terms of explicit Pfaffian formulae.

The distinction between spin-symmetric and spin-antisymmetric pattern fields is both natural and important in the CFT framework: the spin and energy fields are the most elementary instances of spin-antisymmetric and spin-symmetric local fields respectively. The space of lattice local fields is a vector space that can be decomposed into the direct sum of fields that are symmetric and antisymmetric under spin flip.

The above theorem proves the aforementioned conjecture in the following specific case: it allows one to study the scaling limit of the one-point function of $\delta^{-D} \Phi_{\delta}^{F}$ for $D \leq 1$ in simply-connected domains. Following [Hon10,HoSm13,CHI15], we expect the proof naturally extends to multi-field correlations, in order to prove-in full-the conjecture for fields of scaling dimension $D \in\{0,1 / 8,1\}$. Beyond that, the method we use provides a general toolbox to express multipoint correlations of any local lattice field in terms of discrete fermionic observables in discrete domains, and hence to give explicit infinite-volume limits and first order corrections, and reduce the calculation of all subsequent CFT terms to questions in discrete complex analysis (see Applications 1.3.1).

Moreover, the results allow one to study new interesting quantities: for instance, one can estimate spin flip rates for critical Ising Glauber dynamics, including the geometric effects on them up to first order in the mesh size (see Applications 1.3.2).

The results and approach of this paper, as well as the conjectured connection between Ising lattice local fields and CFT, are expected to straightforwardly generalize in two directions. First, the approach can be generalized to arbitrary combinations of,+- and free boundary conditions (the three conformally invariant boundary conditions according 
to CFT [Car84]). Second, the results should extend to more general planar graphs, including, in particular, isoradial graphs.

Let us also point out similar connections between pattern probabilities and conformal invariance obtained by Boutillier in [Bo07] in the context of the dimer model.

The proof relies mainly on discrete complex analytic methods: we use lattice observables, modifying the objects introduced in [Hon 10, CHI15], to connect pattern probabilities with solutions of discrete boundary value problems. This requires precise treatment of multipoint observables on a topological double cover of the lattice with microscopically separated source points at their singularities. We then study the scaling limits of such solutions using discrete complex analysis technique, where, in particular, the neighborhood of the monodromy of the double cover needs to be analyzed delicately. The new techniques introduced for this purpose are: refined analysis of convergence of observables and constructions and characterizations of lattice spinor observables on the slit plane $\left(\mathbb{C} \backslash \mathbb{R}_{>0}\right)_{\delta>0}$, both as limits of finite-volume ones and in terms of discrete harmonic measures (explicitly computed with Fourier techniques).

1.1. Notation. We begin by defining the most important notation, that will be necessary for the statements of the main theorems. We defer a more extensive discussion of the notation used in the proofs to Sect. 1.5.

In this paper, we consider the Ising model with spins on the faces of the graph $\Omega_{\delta}$, a discretization of $\Omega$ of mesh size $\delta>0$. More precisely:

Identify $\mathbb{Z}^{2}$ with the square lattice with vertex set at $\mathbb{Z}+i \mathbb{Z} \subset \mathbb{C}$ and nearest-neighbor edges. Let

$$
\mathbb{C}_{1}:=(1+i) \mathbb{Z}^{2}+1 \quad \text { and } \quad \mathbb{C}_{\delta}=\delta \mathbb{C}_{1}
$$

be the rescaled, rotated and shifted lattice, and its rescaling by a mesh size $\delta>0$, respectively.

For a simply connected open domain $\Omega \subset \mathbb{C}$ bounded by a smooth curve containing 0 (this is easily relaxed to arbitrary simply connected domains [CHI15, Remark 2.10]), define $\Omega_{\delta}$ as the largest connected component of the graph $\Omega \cap \mathbb{C}_{\delta}$. Denote by $\mathcal{V}_{\Omega_{\delta}}$ the set of vertices of $\Omega_{\delta}$, and by $\mathcal{E}_{\Omega_{\delta}}$ the set of edges in $\Omega_{\delta}$. We denote the set of faces of the graph by $\mathcal{F}_{\Omega_{\delta}}$. Whenever needed, we identify the edges in $\mathcal{E}_{\Omega_{\delta}}$ with their midpoints, and the faces in $\mathcal{F}_{\Omega_{\delta}}$ with their centers, such that the origin is identified with a face.

1.1.1. Ising model An Ising configuration $\sigma$ is an assignment of \pm 1 spins to the faces in $\mathcal{F}_{\Omega_{\delta}}$. We consider the critical Ising model on $\mathcal{F}_{\Omega_{\delta}}$ with plus boundary conditions, given by,

$$
\mathbb{P}_{\Omega_{\delta}}(\sigma)=\mathbb{P}_{\Omega_{\delta}}^{+}(\sigma) \propto e^{-\beta_{c} H(\sigma)},
$$

where $\beta_{c}=\frac{1}{2} \ln (\sqrt{2}+1)$ is the critical inverse temperature and $H(\sigma)=-\sum_{x \sim y} \sigma_{x} \sigma_{y}$ with boundary faces fixed to have +1 spin. Let $\mathbb{E}_{\Omega_{\delta}}=\mathbb{E}_{\Omega_{\delta}}^{+}$be its corresponding expectation.

Define the energy density field $(\epsilon(\delta e))_{e \in \mathbb{C}_{1}}$ as follows: for $\delta e \in \mathcal{E}_{\Omega_{\delta}}$ separating faces $\delta f_{1} \sim \delta f_{2}$,

$$
\epsilon(\delta e)=\mu-\sigma_{\delta f_{1}} \sigma_{\delta f_{2}}, \quad \text { where } \quad \mu:=\frac{\sqrt{2}}{2}=\mathbb{E}_{\mathbb{C}_{\delta}}\left[\sigma_{\delta f_{1}} \sigma_{\delta f_{2}}\right]=\mathbb{E}_{\mathbb{C}_{1}}\left[\sigma_{f_{1}} \sigma_{f_{2}}\right] .
$$


Define the spin-weighted energy density field $\left(\epsilon_{[0]}(\delta e)\right)_{e \in \mathbb{C}_{1}}$ on $\mathcal{E}_{\Omega_{\delta}}$ by

$$
\begin{aligned}
\epsilon_{[0]}(\delta e)=\mu_{e}-\sigma_{\delta f_{1}} \sigma_{\delta f_{2}}, \quad \text { where } \quad \mu_{e} & :=\lim _{\Omega \rightarrow \mathbb{C}} \frac{\mathbb{E}_{\Omega_{\delta}}\left[\sigma_{0} \sigma_{\delta f_{1}} \sigma_{\delta f_{2}}\right]}{\mathbb{E}_{\Omega_{\delta}}\left[\sigma_{0}\right]} \\
& =\lim _{\Omega \rightarrow \mathbb{C}} \frac{\mathbb{E}_{\Omega_{1}}\left[\sigma_{0} \sigma_{f_{1}} \sigma_{f_{2}}\right]}{\mathbb{E}_{\Omega_{1}}\left[\sigma_{0}\right]}
\end{aligned}
$$

where the limit is trivially independent of $\delta$ and exists for every $e \in \mathbb{C}_{1}$ by Theorem 1.2 (see Fig. 8 for some exact values). Given a set of edges $\mathcal{B} \subset \mathcal{E}_{\mathbb{C}_{1}}$, we write

$$
\epsilon(\delta \mathcal{B}):=\prod_{e \in \mathcal{B}} \epsilon(\delta e) \quad \text { and analogously } \quad \epsilon_{[0]}(\delta \mathcal{B}):=\prod_{e \in \mathcal{B}} \epsilon_{[0]}(\delta e) .
$$

1.2. Main results. In this section we present the main results. By translation, it suffices to consider the statistics of fields centered at $x=0$. For a collection $\mathcal{B}=\left\{e_{1}, \ldots, e_{n}\right\} \subset$ $\mathcal{E}_{\mathbb{C}_{1}}$, consider the spin-symmetric field $\epsilon(\delta \mathcal{B})=\epsilon\left(\delta e_{1}\right) \cdots \epsilon\left(\delta e_{n}\right)$, i.e., the product of energy densities on a collection of edges around $x$, and the spin-antisymmetric field $\sigma_{0} \epsilon_{[0]}(\delta \mathcal{B})=\sigma_{0} \epsilon_{[0]}\left(\delta e_{1}\right) \cdots \epsilon_{[0]}\left(\delta e_{n}\right)$. If $\mathbf{A}, \mathbf{B}$ are anti-symmetric square matrices of the same dimensions, define the directional derivative of the Pfaffian $\operatorname{Pf}(\mathbf{B})$ (defined in (4.6)) by

$$
D_{\mathbf{A}} \operatorname{Pf}(\mathbf{B})=\lim _{t \downarrow 0} \frac{\operatorname{Pf}(\mathbf{B}+t \mathbf{A})-\operatorname{Pf}(\mathbf{B})}{t} .
$$

For spin-symmetric and spin-antisymmetric fields, we obtain the following two convergence results:

Theorem 1.1 (Spin-symmetric correlations). Let $\mathcal{B}=\left\{e_{1}, \ldots, e_{n}\right\} \subset \mathcal{E}_{\mathbb{C}_{1}}$. There exist explicit, real-valued anti-symmetric $2 n \times 2 n$ matrices $\mathbf{F}^{\mathcal{B}}$ and $\mathbf{E}^{\mathcal{B}}$, such that as $\delta \rightarrow 0$,

$$
\mathbb{E}_{\Omega_{\delta}}[\epsilon(\delta \mathcal{B})]=(-2)^{n} \cdot \operatorname{Pf}\left(\mathbf{F}^{\mathcal{B}}\right)+(-2)^{n} \cdot \delta \cdot r_{\Omega}^{-1}(0) \cdot D_{\mathbf{E}^{\mathcal{B}}} \operatorname{Pf}\left(\mathbf{F}^{\mathcal{B}}\right)+o(\delta),
$$

where $r_{\Omega}(z)$ is the conformal radius of $\Omega$ seen from $z \in \Omega$ (i.e. $r_{\Omega}(z)=\left|\varphi^{\prime}(0)\right|$ where $\varphi: \mathbb{D} \rightarrow \Omega$ is the conformal map such that $\varphi(0)=z$ ).

Theorem 1.2 (Spin-antisymmetric correlations). Let $\mathcal{B}=\left\{e_{1}, \ldots, e_{n}\right\} \subset \mathcal{E}_{\mathbb{C}_{1}}$. The limits $\mu_{e}$ defined in (1.1) exist for everye $\in \mathcal{E}_{\mathbb{C}_{1}}$ and are given explicitly. There exist explicit anti-symmetric $2 n \times 2 n$ matrices $\mathbf{F}_{[0]}^{\mathcal{B}}$ and $\mathbf{E}_{[0]}^{\mathcal{B}}$, the former being real-valued, such that as $\delta \rightarrow 0$,

$$
\begin{aligned}
\frac{\mathbb{E}_{\Omega_{\delta}}\left[\sigma_{0} \epsilon_{[0]}(\delta \mathcal{B})\right]}{\mathbb{E}_{\Omega_{\delta}}\left[\sigma_{0}\right]}=(-2)^{n} \cdot \operatorname{Pf}\left(\mathbf{F}_{[0]}^{\mathcal{B}}\right)+(-2)^{n} \cdot \delta \\
\cdot \operatorname{Re}\left[-\left.\frac{1}{4} \partial_{z} \log r_{\Omega}(z)\right|_{z=0} \cdot D_{\mathbf{E}_{[0]}^{\mathcal{B}} \operatorname{Pf}}\left(\mathbf{F}_{[0]}^{B}\right)\right]+o(\delta),
\end{aligned}
$$

where $z=x+i y$ and $\partial_{z}=\frac{1}{2}\left(\partial_{x}-i \partial_{y}\right)$.

Remark. Theorems 1.1 and 1.2 yield that the infinite-volume limits of $\mathbb{E}_{\Omega_{\delta}}\left[\sigma_{0} \epsilon_{[0]}(\delta \mathcal{B})\right] /$ $\mathbb{E}_{\Omega_{\delta}}\left[\sigma_{0}\right]$ and $\mathbb{E}_{\Omega_{\delta}}[\epsilon(\delta \mathcal{B})]$ exist and are given explicitly by 


$$
\begin{array}{r}
\lim _{\Omega \rightarrow \mathbb{C}} \mathbb{E}_{\Omega_{\delta}}[\epsilon(\delta \mathcal{B})]=(-2)^{n} \cdot \operatorname{Pf}\left(\mathbf{F}^{\mathcal{B}}\right), \quad \text { and } \\
\lim _{\Omega \rightarrow \mathbb{C}} \frac{\mathbb{E}_{\Omega_{\delta}}\left[\sigma_{0} \epsilon_{[0]}(\delta \mathcal{B})\right]}{\mathbb{E}_{\Omega_{\delta}}\left[\sigma_{0}\right]}=(-2)^{n} \cdot \operatorname{Pf}\left(\mathbf{F}_{[0]}^{\mathcal{B}}\right) .
\end{array}
$$

For spin pattern fields, our results translate to the following:

Corollary 1.3 (Conformal invariance of pattern probabilities). Let $\mathcal{F}$ be a finite connected collection of faces of $\mathbb{C}_{1}$ including 0 . For any $\rho \in\{ \pm 1\}^{\mathcal{F}}$ we have:

$$
\begin{gathered}
\delta^{-1}\left(\mathbb{P}_{\Omega_{\delta}}\left[\left.\sigma\right|_{\delta \mathcal{F}} \in\{ \pm \rho\}\right]-\mathbb{P}_{\mathbb{C}_{1}}[\sigma \mid \mathcal{F} \in\{ \pm \rho\}]\right) \underset{\delta \rightarrow 0}{\longrightarrow}\langle\langle\mathcal{F},\{ \pm \rho\}\rangle\rangle_{\Omega}, \\
\delta^{-1 / 8}\left(\mathbb{P}_{\Omega_{\delta}}\left[\left.\sigma\right|_{\delta \mathcal{F}}=\rho\right]-\mathbb{P}_{\mathbb{C}_{1}}[\sigma \mid \mathcal{F}=\rho]\right) \underset{\delta \rightarrow 0}{\longrightarrow}\langle\langle\mathcal{F}, \rho\rangle\rangle_{\Omega}^{\prime},
\end{gathered}
$$

where the functions $\langle\langle\cdot\rangle\rangle_{\Omega}$ and $\langle\langle\cdot\rangle\rangle_{\Omega}^{\prime}$ depend only on $\Omega$, and where:

- infinite-volume limits $\mathbb{P}_{\mathbb{C}_{1}}[\sigma \mid \mathcal{F} \in\{ \pm \rho\}]=\lim _{\Omega \rightarrow \mathbb{C}} \mathbb{P}_{\Omega_{1}}[\sigma \mid \mathcal{F} \in\{ \pm \rho\}]$ and $\mathbb{P}_{\mathbb{C}_{1}}[\sigma \mid \mathcal{F}=$ $\rho]$ are explicit.

- $\langle\langle\mathcal{F},\{ \pm \rho\}\rangle\rangle_{\Omega}$ and $\langle\langle\mathcal{F}, \rho\rangle\rangle_{\Omega}^{\prime}$ are explicit and are such that for the map $\varphi: \mathbb{D} \rightarrow \Omega$ as in Theorem 1.1,

$$
\langle\langle\mathcal{F},\{ \pm \rho\}\rangle\rangle_{\Omega}=r_{\Omega}^{-1}(0)\langle\langle\mathcal{F},\{ \pm \rho\}\rangle\rangle_{\mathbb{D}}, \quad \text { and } \quad\langle\langle\mathcal{F}, \rho\rangle\rangle_{\Omega}^{\prime}=r_{\Omega}^{-\frac{1}{8}}(0)\langle\langle\mathcal{F}, \rho\rangle\rangle_{\mathbb{D}}^{\prime} .
$$

As a result of Corollary 1.3, our results include explicit expressions for all the finitedimensional distributions of $\mathbb{P}_{\mathbb{Z}^{2}}$ as finite linear combinations of a certain Fourier integral given in Theorem 3.21.

1.3. Applications. In this subsection, we briefly detail three applications of our results: the lattice local field conjecture of the introduction, relations between Markov chain dynamics flip rates and the geometry of the domain where the dynamics live, and explicit computations of pattern probabilities under the Gibbs measure.

1.3.1. Lattice local fields and CFT Returning to the conjectured Ising-CFT correspondence in terms of lattice fields, we observe that any lattice local field $\Phi_{\delta}^{F}(x)$ is such that $F$ can be expressed as a linear combination of indicator functions of spin-pattern events in a microscopic neighborhood of $x$; a spin-symmetric lattice local field can in particular be written in terms of indicators of spin-symmetric pattern events.

Then Theorems 1.1-1.2 give the infinite-volume limits, and first-order CFT corrections of the one point function of any lattice local field $\Phi_{\delta}^{F}(x)$ in terms of those of spin-symmetric and spin-antisymmetric pattern fields, whose one-point functions can be obtained explicitly. We believe extending this to multi-field correlations of fields with scaling dimension $D \leq 1$ should carry over from [Hon10, CHI15].

In the other direction, though our main statements only go up to first-order corrections $\left(\delta^{1 / 8}\right.$ or $\left.\delta\right)$, the methods of this paper can, in principle, be employed to reduce the computation of higher order CFT corrections to correlations of any local lattice field to questions of discrete complex analysis. Of course, then obtaining the necessary sharper discrete complex analytic expansions is itself a major obstacle to the extension of such results. All the same, using the present framework along with, hypothetically, improved discrete complex analysis asymptotics, should yield that all Ising lattice local fields are, as conjectured in the Introduction, either zero in correlations, or have scaling dimensions $\Delta \in \mathbb{N} \cup\left(\mathbb{N}+\frac{1}{8}\right)$, as predicted from the Ising CFT. 
1.3.2. Local Markov chain dynamics There are a number of Markov chain dynamics for which the Ising measure is the stationary measure; as a result, an efficient way to sample the Ising model is to run such a Markov chain for long times.

Of particular importance are local dynamics, such as the Glauber dynamics, where one picks a spin at random, and flips it with a probability given by the state of spins in a microscopic neighborhood of it (the simplest one using only the four neighbors). At critical temperature interesting dynamical behavior arises (see [LuS112]). In particular, as our results explain, the geometry of the domain $\Omega$ has a measurable (i.e. inverse polynomial-sized) effect on the local dynamics of the Markov chain.

For such a dynamics, our results allow one to describe, once we are at equilibrium, the relevant observables to compute the average flip rates of the system: those are indeed given in terms of the occurence probabilities of various spin patterns (typically spinsymmetric patterns).

In particular, Corollary 1.3 gives us the following: at criticality, for any Glauber dynamics (see e.g., [LuS112, Section 2.1]), we can derive exact information about spinsymmetric pattern probabilities, how they behave at constant order, and how the firstorder correction depends on a geometric quantity.

Knowing the long-term history of a Glauber dynamics in a microscopic neighborhood of a point enables the computation of various spin pattern probabilities and hence lattice local field one-point functions. Higher order corrections of these terms in turn give geometric information beyond the conformal radius of the domain. A particularly interesting question, for which our results provide relevant tools, is the following one, due to Benjamini (private communication to the second author): does the complete (i.e., unbounded in time) knowledge of the flip history of a single spin allow one to recover the shape of the domain $\Omega$, up to isometry?

1.3.3. Explicit computations Explicit calculation of infinite-volume limits and finitesize corrections of pattern probabilities in the critical Ising model is of general interest, and may be particularly useful for the program of Application 1.3.2. Such computation requires the explicit matrices of Theorems 1.1-1.2, which are expressed in terms of the full-plane fermion and spin-fermion observables: some values of the former are given in e.g., [Ken00]; we characterize the latter as a Fourier integral (see Theorem 3.21) and give some of its values in Fig. 4. In particular, the entries of the matrices in Theorems 1.1-1.2 are given as finite linear combinations of a slit-plane harmonic measure, whose values are explicitly computable as a Fourier integral.

We present an example computation of the infinite-volume limit and first-order conformal correction of $\mathbb{E}_{\Omega_{\delta}}\left[\sigma_{0} \sigma_{2 \delta}\right]$ in Corollary C.1 of Appendix C, where, since the spins live on the rotated square lattice, this is a pair of diagonally "adjacent" spins. The first and second order corrections to this term, and their representation in terms of discrete complex analysis will be used in the Ising stress tensor on the lattice level (see [BeHo18] and, for an alternative approach to the stress tensor, [CGS17]).

As a computation of spin-antisymmetric fields, Corollary C.2 gives the values of the infinite-volume limit and conformal correction to the spin weighted "L"-shaped correlation $\mathbb{E}_{\Omega_{\delta}}\left[\sigma_{0} \sigma_{(1+i) \delta} \sigma_{2 \delta}\right] / \mathbb{E}_{\Omega_{\delta}}\left[\sigma_{0}\right]$.

1.4. Proof outline. In this subsection we outline the strategy for proving our main results: Theorems 1.1-1.2. The proof combines ideas from [Hon10,CHI15], and we try to focus the outline on the places where substantial new ingredients are needed. The steps in proving the main theorems broadly consist of the following. 
Section 2 defines standard concepts in discrete complex analysis as well as the discrete Riemann boundary value problems solved by certain discrete observables. Section 3 begins by defining the two-point discrete fermion and spin-fermion (given by $(\alpha, \zeta) \mapsto$ $F_{\Omega_{\delta}}^{\alpha, \zeta}$ and $F_{\left[\Omega_{\delta}, 0\right]}^{\alpha, \zeta}$ respectively) via the low-temperature expansion of the Ising model and disorder lines, as well as their full-plane analogues.

- In Sect. 3.1, we define the bounded domain observables on $\Omega_{\delta}$, as previously defined in [HoSm13, CHI15].

- In Sect. 3.2, we introduce their full-plane analogues: the full plane fermion $H_{\mathbb{C}_{1}}^{\alpha}(z)$ is given explicitly by a formula due to Kenyon. For the special value of $\alpha_{0}=\frac{1}{2}$, the full plane spin-fermion $H_{\left[\mathbb{C}_{1}, 0\right]}^{\alpha_{0}}(z)$ was given by [CHI15]. Here, we prove existence of the infinite-volume limit of the spin-fermion $H_{\left[\mathbb{C}_{1}, 0\right]}^{\alpha}$ for every $\alpha$, and express it as a finite linear combination of discrete harmonic measures on $\mathbb{C}_{1} \backslash \mathbb{R}_{>0}$. Moreover, we give an explicit representation formula using Fourier techniques for this discrete harmonic measure (see Theorem 3.21), allowing computation of $H_{\left[\mathbb{C}_{1}, 0\right]}^{\alpha}(z)$ for arbitrary $\alpha$.

Section 4 defines and analyzes $n$-point analogues of the two-point fermion and spinfermion. This section is notationally heavy, but many of its proofs are straightforward adaptations of proofs in [Hon10].

- We first recall the multipoint fermion defined in [Hon10] and slightly, but crucially, generalize its properties to the setting where its arguments are permitted to be adjacent edges.

- Motivated by this definition, we consider a multipoint version of the spin-fermion observable in Definition 4.2 and prove that the same properties hold after minor modifications.

- In Proposition 4.13 we relate specific values taken by the multipoint fermion and spin-fermion to the $n$-point spin-symmetric and spin-antisymmetric correlations of Theorems 1.1-1.2.

- These results allow us to arrive at the spin-antisymmetric analogues of the Pfaffian formulae of [Hon10], connecting spin-antisymmetric $n$-point Ising correlations to the Pfaffian of a matrix with entries consisting only of the two-point spin-fermion (see Proposition 4.17).

Section 5 defines two-point continuous observables, and proves that they are the renormalized scaling limits (as $\delta \rightarrow 0$ ) of the discrete two-point observables.

- In Sect. 5.2 we introduce the continuous analogues of the discrete Riemann boundary value problem defined in Sect. 2 and give their full-plane solutions and bounded domain solutions $h_{\Omega}^{\alpha}$ and $h_{[\Omega, 0]}^{\alpha}$. Again this was already done for the fermion in [Hon10,HoSm13] and for the spin-fermion in the particular case of $h_{[\Omega, 0]}^{\alpha=\frac{1}{2}}[\mathrm{CHI} 15]$; extra care is needed in constructing the continuous bounded domain and full-plane spin-fermions for arbitrary source point $\alpha$.

- The heart of Sect. 5, Sect. 5.4 proves convergence of a rescaled, renormalized discrete spin-fermion to a conformally covariant quantity obtained by Taylor expanding $h_{[\Omega, 0]}^{\alpha}$, for arbitrary $\alpha$. In adapting the proof of convergence in [CHI15] to the case where $\alpha \neq \frac{1}{2}$, we require refined analysis of the observables near their branch points and singularities. Here we encounter some discrete complex analytic peculiarities regarding discretizations of the function $i \sqrt{z}$ which are independently interesting.

Section 6 combines the Pfaffian formulae of Sect. 4.4 expressing $n$-point correlations in terms of two-point discrete observables, with the convergence results of Sect.5, to prove Theorems 1.1-1.2. 
In Appendix A, we prove the validity of our explicit construction of the discrete harmonic measure on $\mathbb{C}_{1} \backslash \mathbb{R}_{>0}$ and provide a recursive formula to obtain its value at any lattice point as a finite linear combination of Fourier integrals. In Appendix B, Proposition B.1, we provide a combinatorial proof of the well-definedness of the discrete multipoint spin-fermion we introduce in Definition 4.2. As mentioned earlier, Appendix C consists of explicit computations of the infinite-volume limit and first-order correction of the correlations of two diagonally adjacent spins and three spins in an "L" shape.

1.5. Extra notation and glossary. We now introduce extended notation that will be used globally throughout the paper. This notation mimics very closely that of [CHI15], and we try to make note of places where our conventions differ.

\subsubsection{Relevant constants The following constants will recur throughout the paper.}

\begin{tabular}{lll}
\hline$\beta_{c}=\frac{1}{2} \log (1+\sqrt{2})$ & $\mu=\frac{\sqrt{2}}{2}$ & $\lambda=e^{i \pi / 4}$ \\
\hline
\end{tabular}

1.5.2. Graph notation We list below the additional graph notation that will be used throughout the paper.

- For two adjacent vertices $a, b \in \mathcal{V}_{\Omega_{\delta}}$ the edge $e=\{a, b\}$ is identified with the line segment in $\Omega$ connecting $a$ and $b$; we define the set of medial vertices $\mathcal{V}_{\Omega_{\delta}}^{m}$ as the set of edge midpoints; given an edge $e \in \mathcal{E}_{\Omega_{\delta}}$, we denote its midpoint by $m(e) \in \mathcal{V}_{\Omega_{\delta}}^{m}$, and conversely for $m \in \mathcal{V}_{\Omega_{\delta}}^{m}$ the corresponding edge $e(m) \in \mathcal{E}_{\Omega_{\delta}}$.

- We call corners the points that are at distance $\delta / 2$ from the vertices in one of the four $\pm 1, \pm i$ directions. Following [CHI15], we set

$\mathcal{V}_{\Omega_{\delta}}^{1}:=\mathcal{V}_{\Omega_{\delta}}+\frac{\delta}{2}, \quad \mathcal{V}_{\Omega_{\delta}}^{i}:=\mathcal{V}_{\Omega_{\delta}}-\frac{\delta}{2}, \quad \mathcal{V}_{\Omega_{\delta}}^{\lambda}:=\mathcal{V}_{\Omega_{\delta}}-\frac{i \delta}{2}, \quad$ and $\mathcal{V}_{\Omega_{\delta}}^{\bar{\lambda}}:=\mathcal{V}_{\Omega_{\delta}}+\frac{i \delta}{2}$.

The set of corners $\mathcal{V}_{\Omega_{\delta}}^{c}$ is the union $\mathcal{V}_{\Omega_{\delta}}^{1} \cup \mathcal{V}_{\Omega_{\delta}}^{i} \cup \mathcal{V}_{\Omega_{\delta_{\delta}}}^{\lambda} \cup \mathcal{V}_{\Omega_{\delta}}^{\bar{\lambda}}$.

- The domain of definitions for most discrete functions in the following sections is the set of both corners and medial vertices, or $\mathcal{V}_{\Omega_{\delta}}^{c m}:=\mathcal{V}_{\Omega_{\delta}}^{c} \cup \mathcal{V}_{\Omega_{\delta}}^{m}$. We declare a medial vertex and a corner adjacent if they are $\frac{\delta}{2}$ apart from each other.

- The boundary faces $\partial \mathcal{F}_{\Omega_{\delta}}$, boundary medial vertices $\partial \mathcal{V}_{\Omega_{\delta}}^{m}$, boundary edges in $\partial \mathcal{E}_{\Omega_{\delta}}$ are those faces, medial vertices, and edges in $\mathbb{C}_{\delta}$ that are incident to but not contained in $\mathcal{F}_{\Omega_{\delta}}, \mathcal{V}_{\Omega_{\delta}}^{m}$, and $\mathcal{E}_{\Omega_{\delta}}$.

- Given a boundary edge $z$ (resp., boundary medial vertex), we define the unit normal outward vector $v_{z}$ as the unit vector in the direction of the vertex in $\mathbb{C} \backslash \Omega$ viewed from the vertex inside $\Omega$.

1.5.3. Graph lifts to the double cover For the discrete functions with monodromy which will be introduced in Sect. 3, we work with graphs lifted to the double cover $[\Omega, 0]$ of $\Omega \backslash\{0\}$.

- We denote by $[\mathbb{C}, 0]$ the double cover of the plane $\mathbb{C}$ ramified at 0 , i.e. the surface on which the function $z \mapsto \sqrt{z} \in \mathbb{C} \backslash\{0\}$ is naturally defined; above each point of $\mathbb{C} \backslash\{0\}$ lie exactly two points of $[\mathbb{C}, 0]$. We will sometimes use $z \in[\mathbb{C}, 0]$ to refer to its projection on $\mathbb{C}$ in unambiguous cases. 
- If $z_{1}, z_{2} \in[\mathbb{C}, 0]$ are two points above $w_{1}, w_{2} \in \mathbb{C} \backslash\{0\}$ such that $\operatorname{Re}\left(\frac{w_{1}}{w_{2}}\right)>0$, we say that they are on the same sheet if $\operatorname{Re}\left(\frac{\sqrt{z_{1}}}{\sqrt{z_{2}}}\right)>0$ and that they are on opposite sheets if $\operatorname{Re}\left(\frac{\sqrt{z_{1}}}{\sqrt{z_{2}}}\right)<0$.

- If $z \in[\mathbb{C}, 0]$ lies above $w \in \mathbb{C} \backslash\{0\}$, we define $z+x \in[\mathbb{C}, 0]$, for $x \in \mathbb{C}$ small enough, as the point above $w+x$ that is on the same sheet as $z$.

- We define complex conjugation on the double cover by conjugating the square root. In other words, the complex conjugate $\bar{z}$ of $z \in[\mathbb{C}, 0]$ is defined by the condition that $\sqrt{\bar{z}}=\overline{\sqrt{z}}$.

- We call functions with monodromy -1 around 0 spinors; these are naturally defined on $[\mathbb{C}, 0]$.

- We denote by $\left[\Omega_{\delta}, 0\right]$ the double cover of $\Omega_{\delta}$ ramified at 0 ; in other words, the vertices, medial vertices, and corners get lifted from $\Omega_{\delta}$ to yield the lifted vertex, edge and corner sets. We use similar notations for the lifted vertex, edge, and corner sets as above by replacing $\Omega_{\delta}$ with $\left[\Omega_{\delta}, 0\right]$. Moreover, $\left[\Omega_{\delta}, 0\right]$ can be naturally viewed as a subgraph of $\left[\mathbb{C}_{\delta}, 0\right]$ via the natural inclusion $[\Omega, 0] \subset[\mathbb{C}, 0]$.

- Identify the branches of the double cover [C, 0$]$ using the function $\sqrt{z}$ as follows:

$\mathbb{X}:=\mathbb{C} \backslash \mathbb{R}_{<0} \quad$ with $\mathbb{X}^{+}=\{z \in[\mathbb{C}, 0]: \operatorname{Re}(\sqrt{z})>0\}$ and $\mathbb{X}^{-}=\{z \in[\mathbb{C}, 0]: \operatorname{Re}(\sqrt{z})<0\}$

$\mathbb{Y}:=\mathbb{C} \backslash \mathbb{R}_{>0} \quad$ with $\mathbb{Y}^{+}=\{z \in[\mathbb{C}, 0]: \operatorname{Im}(\sqrt{z})>0\}$ and $\mathbb{Y}^{-}=\{z \in[\mathbb{C}, 0]: \operatorname{Im}(\sqrt{z})<0\}$

On the discrete level, define the lift of $\mathcal{V}_{\Omega_{\delta}}^{1}$ to $\mathbb{X}^{ \pm}$as $\mathbb{X}_{\delta}^{ \pm}$, and the lift of $\mathcal{V}_{\Omega_{\delta}}^{i}$ to $\mathbb{Y}^{ \pm}$ as $\mathbb{Y}_{\delta}^{ \pm}$.

1.5.4. Orientations We define orientations and s-orientations for corners and medial vertices.

- Given an edge $e=\{a, b\} \in \mathcal{E}_{\Omega_{\delta}}$, we denote the two orientations of $e$ by the complex numbers $(a-b) /|a-b|$ and $(b-a) /|b-a|$. We can subdivide $e$ into two half edges $\{a, m(e)\}$ and $\{m(e), b\}$; their union is identified with the whole edge $e$. An orientation $o=o(e)$ is compatible with a half edge $\{a, m(e)\}$ if $a-m(e)$ points to the same direction: i.e., $o=(a-m(e)) /|a-m(e)|$.

- We call an oriented medial vertex and denote by $m^{o}$ an edge midpoint $m(e)$ together with an orientation of the edge $e$. We denote by $\mathcal{V}_{\Omega_{\delta}}^{o}$ the set of oriented medial vertices.

- For a corner $c$, we define its orientation $o=o(c)$ as the complex number $(v-c) /|v-c|$, where $v$ is the nearest vertex to $c$.

- To an orientation $o$ we further associate two $s$-orientations corresponding to the two choices of square root for $o$; we often denote an s-orientation by $(\sqrt{o})^{2}$, indicating this choice.

1.5.5. Glossary For the reader's convenience, we compile some of the most important terminology and quantities used across the paper (see also Fig. 2 for the graph notation). We first recall the various graphs we work with: if $\Omega$ is a simply-connected smooth domain containing the origin and $\bar{\Omega}$ is its complex conjugate,

$\begin{array}{lllll}\mathbb{C}_{1}=(1+i) \mathbb{Z}^{2}+1 & \mathbb{C}_{\delta}=\delta \mathbb{C}_{1} & \Omega_{\delta}=\Omega \cap \mathbb{C}_{\delta} & \Lambda_{\delta}=\Omega_{\delta} \cap(\bar{\Omega})_{\delta} & a_{0}=\frac{1}{2}\end{array}$




$$
\begin{gathered}
\mathcal{V}_{\Omega_{\delta}}=\bullet \\
\mathcal{V}_{\Omega_{\delta}}^{\tau}={ }^{2} \tau \\
\mathcal{V}_{\Omega_{\delta}}^{c}=\bullet \\
\mathcal{V}_{\Omega_{\delta}}^{m}=\circ \\
\mathcal{V}_{\Omega_{\delta}}^{c m}=\bullet, \circ \\
\mathcal{F}_{\Omega_{\delta}}=\times
\end{gathered}
$$

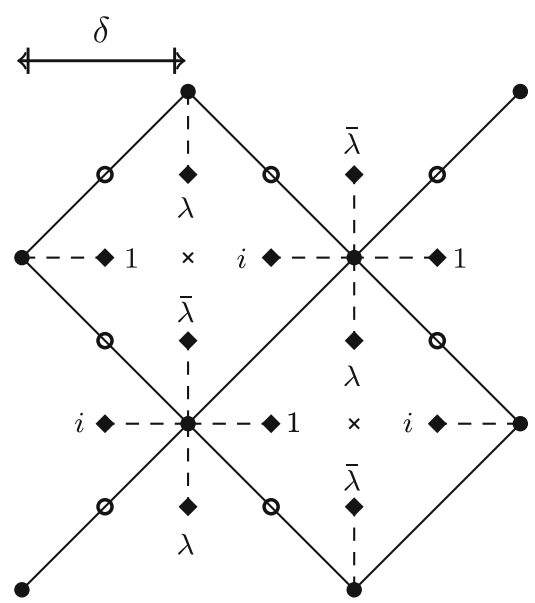

Fig. 2. The graph notation on discretizations of $\Omega$ where $\tau \in\{1, i, \lambda, \bar{\lambda}\}$; the notation on $\left[\Omega_{\delta}, 0\right]$ is analogously defined

and the quantities $\left[\mathbb{C}_{1}, 0\right],\left[\Omega_{\delta}, 0\right]$, etc. are analogously defined on the ramified plane $[\mathbb{C}, 0]$. When proofs are independent of the choice, we let $D_{\delta}$ denote either of $\Omega_{\delta}$ or $\left[\Omega_{\delta}, 0\right]$.

The domain-dependent quantities of interest match with [CHI15] and read as follows for fixed $z \in \Omega$.

\begin{tabular}{lll}
\hline \multicolumn{2}{l}{ Geometric quantities } \\
\hline$\varphi(\omega)$ & conformal map $\varphi: \mathbb{D} \rightarrow \Omega$ with $\varphi(0)=z$ & \\
$r_{\Omega}(z)$ & $r_{\Omega}(z)=\left|\varphi^{\prime}(0)\right|$ & conformal radius of $z$ \\
$\mathcal{A}_{\Omega}$ & $\mathcal{A}_{\Omega}=-\left.\frac{1}{4} \partial_{z} \log r_{\Omega}(z)\right|_{z=0}$ & Remark 5.13 \\
\hline
\end{tabular}

In what follows, we present the notation for the fundamental observables we deal with; in order to reduce multipoint observables to such two-point functions via Pfaffian relations we need much heavier notation, that is restricted to Sect. 4. In the sequel, $a, z$ will be medial vertices or corners and $\alpha$ and $\zeta$ will be their s-oriented counterparts, e.g., $\alpha=a^{(\sqrt{o})^{2}}$.

\begin{tabular}{lll}
\hline Fermion observables & & \\
\hline$F_{\Omega_{\delta}, \zeta}^{\alpha, j}$ & discrete real fermion & Definition 3.2 \\
$H_{\Omega_{\delta}}^{\alpha}(z)$ & discrete complexified fermion & Definition 3.8 \\
$F_{\mathbb{C}_{1}^{\alpha \zeta}}^{\alpha}$ & discrete full-plane fermion & Theorem 3.13 \\
$H_{\mathbb{C}_{1}}^{\alpha}(z)$ & discrete full-plane complexified fermion & Proposition 3.14 \\
$f_{\Omega}, h_{\Omega}, f_{\mathbb{C}}, h_{\mathbb{C}}$ & continuous counterparts of the above & Definitions 5.6, 5.8 \\
$F_{\Omega_{\delta}}^{\dagger}, H_{\Omega_{\delta}}^{\dagger}, f_{\Omega}^{\dagger}, h_{\Omega}^{\dagger}$ & e.g., $F_{\Omega_{\delta}}^{\dagger, \alpha, \zeta}=F_{\Omega_{\delta}}^{\alpha, \zeta}-F_{\mathbb{C}_{\delta}}^{\alpha, \zeta}$ & \\
\hline
\end{tabular}


The notation for the spin-fermions is analogous to the above, but on the respective double covers.

\begin{tabular}{lll}
\hline Spinor observables & & \\
\hline$F_{\left[\Omega_{\delta}, 0\right]}^{\alpha, \zeta}$ & discrete real spin-fermion & Definition 3.3 \\
$H_{\left[\Omega_{\delta}, 0\right]}^{\alpha}(z)$ & discrete complexified spin-fermion & Definition 3.9 \\
$F_{\left[\mathbb{C}_{1}, 0\right]}^{\alpha, \zeta}$ & discrete full-plane spin-fermion & Theorem 3.15, item (E) \\
$H_{\left[\mathbb{C}_{1}, 0\right]}^{\alpha}(z)$ & discrete full-plane complexified spin-fermion & Proposition 3.19 \\
$S_{\left[\Omega_{\delta}, 0\right]}^{\alpha}(z), A_{\left[\Omega_{\delta}, 0\right]}^{\alpha}(z)$ & symmetrized and anti-symmetrized observables & Definition 3.11 \\
$G_{\left[\mathbb{C}_{1}, 0\right]}(z), \tilde{G}_{\left[\mathbb{C}_{1}, 0\right]}^{ \pm}(z)$ & auxiliary functions & Definitions 3.25 \\
$C_{\alpha}=C_{a^{o}}$ & $C_{\alpha}=-\operatorname{Re}\left[i \sqrt{o}\left(\tilde{G}_{\left[\mathbb{C}_{1}, 0\right]}^{+}-\tilde{G}_{\left[\mathbb{C}_{1}, 0\right]}^{-}\right)(a)\right]$ & Corollary 5.19 \\
\hline
\end{tabular}

The lower-case versions of the spin-fermions above again are their continuous counterparts, and when there is a $\uparrow$ superscript, that denotes the difference of the bounded-domain and full-plane spin-fermions.

\section{Discrete Complex Analysis}

In this section, we review basic notions of discrete complex analysis that will be useful in this paper. We use discrete complex analysis for the following:

- To relate the Ising correlations to Pfaffians of fermion and spin-fermion observables.

- To obtain explicit formulae for the full-plane observables.

- To establish the convergence of the two-point observables and study their local behavior.

\subsection{S-holomorphicity.}

Definition 2.1. Associate to each corner $c \in \mathcal{V}_{\Omega_{\delta}}^{\tau}$ with $\tau \in\{1, i, \lambda, \bar{\lambda}\}$, the line $l(c):=$ $\tau \mathbb{R}$ in the complex plane. A function $H_{\delta}$ defined on corners and medial vertices of a discrete domain $\Omega_{\delta}$ is said to be s-holomorphic at a corner $c \in \mathcal{V}_{\Omega_{\delta}}^{\tau}$ if for any adjacent medial vertex $a \in \mathcal{V}_{\Omega_{\delta}}^{m}$ we have

$$
H_{\delta}(c)=\mathrm{P}_{l(c)}\left[H_{\delta}(a)\right]:=\frac{1}{2}\left(H_{\delta}(a)+\tau^{2} \bar{H}_{\delta}(a)\right),
$$

where $\mathrm{P}_{l(c)}$ denotes orthogonal projection in the complex plane onto the line $l(c)$. The function $H_{\delta}$ is said to be s-holomorphic at a medial vertex $a \in \mathcal{V}_{\Omega_{\delta}}^{m}$ if Eq. (2.1) holds for all corners $c$ adjacent to $a$. A function is said to be s-holomorphic on $\Omega_{\delta}$ if it is s-holomorphic at every $c \in \mathcal{V}_{\Omega_{\delta}}^{c}$.

Remark 2.2. If a function $H_{\delta}$ is s-holomorphic on a discrete domain, then it is purely real on the corners of type 1 and purely imaginary on the corners of type $i$. We call respective restrictions to those corners the real part and the imaginary part of $H_{\delta}$. 
Remark 2.3. S-holomorphicity implies usual discrete holomorphicity of the real and imaginary parts, defined by a lattice version of Cauchy-Riemann equations [Smi10]. If $H_{\delta}$ is s-holomorphic, then the following discrete derivative vanishes:

$$
\bar{\partial}_{\delta} H_{\delta}(x):=H_{\delta}\left(x+\frac{\lambda \delta}{\sqrt{2}}\right)-H_{\delta}\left(x-\frac{\lambda \delta}{\sqrt{2}}\right)+i\left(H_{\delta}\left(x-\frac{\bar{\lambda} \delta}{\sqrt{2}}\right)-H_{\delta}\left(x+\frac{\bar{\lambda} \delta}{\sqrt{2}}\right)\right)=0
$$

for $x \in \mathcal{V}_{\Omega_{\delta}}^{\lambda} \cup \mathcal{V}_{\Omega_{\delta}}^{\bar{\lambda}}$. One similarly defines $\partial_{\delta}$ by taking a negative sign in front of $i$. We extend the definition to $x \in \mathcal{V}_{\Omega_{\delta}} \cup \mathcal{F}_{\Omega_{\delta}}$ by setting $\partial_{\delta} H_{\delta}(x):=\partial_{\delta} H_{\delta}\left(x-\frac{i \delta}{2}\right)+\partial_{\delta} H_{\delta}\left(x+\frac{i \delta}{2}\right)$. Note the differences in our definitions compared to their continuous counterparts, as the discrete derivatives are taken in rotated directions (thus differing by a phase factor); however, we will not take direct scaling limits of the operator and this poses no problem.

The information defined on corners of type $1, i$ is enough to recover an s-holomorphic function on $\mathcal{V}_{\Omega_{\delta}}^{\mathrm{cm}}$ : one can start from a discrete holomorphic function defined on corners of type 1 and $i$, reconstruct values on medial vertices based on their projections onto $\mathbb{R}$ and $i \mathbb{R}$, then project to corners of type $\lambda$ and $\bar{\lambda}$ (discrete holomorphicity guarantees well-definedness at those corners); see [CHI15, Remark 3.1].

Definition 2.4. We define the discrete Laplacian $\Delta_{\delta}$ by

$$
\begin{aligned}
\Delta_{\delta} H_{\delta}(x)= & H_{\delta}(x+\delta+i \delta)+H_{\delta}(x-\delta+i \delta)+H_{\delta}(x-\delta-i \delta) \\
& +H_{\delta}(x+\delta-i \delta)-4 H_{\delta}(x) .
\end{aligned}
$$

This quantity makes sense on any discrete domain of rotated square type, for example $\mathcal{V}_{\Omega_{\delta}}^{1}$ or $\mathcal{V}_{\Omega_{\delta}}^{i}$. A function $H_{\delta}$ on such a lattice is said to be discrete harmonic if $\Delta_{\delta} H_{\delta}(x)=0$ for all $x$ at which $\Delta_{\delta} H_{\delta}$ is defined. Analogously, it is discrete sub-harmonic if $\Delta_{\delta} H_{\delta} \geq 0$, and discrete super-harmonic if $\Delta_{\delta} H_{\delta} \leq 0$.

Remark 2.5. The real and imaginary parts of an s-holomorphic function on a planar domain are discrete harmonic on their respective lattices, $\mathcal{V}_{\Omega_{\delta}}^{1}$ and $\mathcal{V}_{\Omega_{\delta}}^{i}$. This is a direct consequence of Remark 2.3: discrete holomorphicity converts discrete outward derivatives from the center point in the Laplacian into discrete derivatives in the angular direction, and going in a closed loop around the center point gives zero.

Remark 2.6. The notions of discrete complex analysis thus far introduced have been defined on the planar domain $\Omega_{\delta}$, but they generalize to $\left[\Omega_{\delta}, 0\right]$ in a straightforward manner since the double cover is locally isomorphic to a planar domain (cf. Sect. 1.5). However, great care is needed in applying Remark 2.5 because, if the center point of the Laplacian is one of the corners on the monodromy face labeled by 0 , the loop around the center point must enclose the monodromy; as a result its lift to $\left[\Omega_{\delta}, 0\right]$ is not closed, and thus discrete holomorphicity does not imply harmonicity at the real and imaginary corners on the face 0 . We may still obtain harmonicity of a discrete holomorphic spinor at one of those two types of corners if we assume in addition that it vanishes at the other, since the sum of discrete derivatives will vanish as though the spinor does not branch at 0 .

2.2. Discrete singularities. Discrete singularities appear as violations of the s-holomorphicity projection relations relations. To study these, we define front and back values at a singularity in order to introduce the notion of discrete residue of a function $H_{\delta}$ at an oriented medial vertex. 
Definition 2.7. Let $H_{\delta}$ be a function defined on an oriented medial vertex $\alpha=a^{o}$ and its adjacent four corners. Let $c_{1}, c_{2}$ be the two corners adjacent to $a$ in the direction of $o$ (i.e., $c_{1}=a+\frac{\sqrt{2} \operatorname{Re}(o) \delta}{2}$ and $\left.c_{2}=a+\frac{\sqrt{2} \operatorname{Im}(o) \delta}{2}\right)$. We define the front value $H_{\delta}\left(\alpha_{+}\right)$as the unique value such that

$$
H_{\delta}\left(c_{1}\right)=\mathrm{P}_{l\left(c_{1}\right)}\left[H_{\delta}\left(\alpha_{+}\right)\right], \quad \text { and } \quad H_{\delta}\left(c_{2}\right)=\mathrm{P}_{l\left(c_{2}\right)}\left[H_{\delta}\left(\alpha_{+}\right)\right] .
$$

Likewise, if $c_{3}, c_{4}$ are the two corners adjacent to $a$ in the direction of $-o$ (i.e., $c_{3}=$ $a-\frac{\sqrt{2} \operatorname{Re}(o) \delta}{2}$ and $\left.c_{4}=a-\frac{\sqrt{2} \operatorname{Im}(o) \delta}{2}\right)$, we set the back value $H_{\delta}\left(\alpha_{-}\right)$as the unique value such that

$$
H_{\delta}\left(c_{3}\right)=\mathrm{P}_{l\left(c_{3}\right)}\left[H_{\delta}\left(\alpha_{-}\right)\right], \quad \text { and } \quad H_{\delta}\left(c_{4}\right)=\mathrm{P}_{l\left(c_{4}\right)}\left[H_{\delta}\left(\alpha_{-}\right)\right] .
$$

Definition 2.8. The discrete residue of $H_{\delta}$ at $\alpha$ is the difference $\operatorname{Res}_{\alpha}\left(H_{\delta}\right):=H_{\delta}\left(\alpha_{+}\right)-$ $H_{\delta}\left(\alpha_{-}\right)$.

By definition $H_{\delta}$ has an s-holomorphic extension to $a$ if and only if the discrete residue is zero at $\alpha$. It is an analog of the residue in the continuous setting in that doing a closed contour sum around $a$ along the edges of the lattice (i.e. summing $H_{\delta}(e) \boldsymbol{e}$ where $\boldsymbol{e}$ is the vector pointing from the start to the end of the edge $e$ on the closed counterclockwise path) will yield $\sqrt{2} o i \delta \operatorname{Res}_{\alpha}\left(H_{\delta}\right)$, for any choice of $o$.

2.3. Discrete Riemann boundary value problems. The key tool for our analysis is the study of discrete Riemann boundary value problems. To prove the convergence of the Ising model observables as the mesh size goes to zero, we will formulate them as the unique solutions to such problems.

Recall that we denote by $\partial \mathcal{V}_{\Omega_{\delta}}^{m}$ the set of boundary medial vertices, by $\nu_{z}$ the outer normal at any $z \in \partial \mathcal{V}_{\Omega_{\delta}}^{m}$, i.e. the orientation at $z$ which points outward from $\Omega_{\delta}$, and $\partial_{\nu_{z}}$ the outer normal difference, i.e. the value on the outer adjacent vertex minus the value on the inner adjacent vertex.

Definition 2.9. We say that a function $H_{\delta}: \mathcal{V}_{\Omega_{\delta}}^{c m} \rightarrow \mathbb{C}$ defined on corners and medial vertices of a discrete domain $\Omega_{\delta}$ is the solution to the discrete Riemann boundary value problem on $\Omega_{\delta}$ with boundary data $f: \partial \mathcal{V}_{\Omega_{\delta}}^{m} \rightarrow \mathbb{C}$ if it is s-holomorphic and $H_{\delta}(z)-$ $f_{\delta}(z) \in \nu_{z}^{-\frac{1}{2}} \mathbb{R}$ for any boundary medial vertex $z \in \partial \mathcal{V}_{\Omega_{\delta}}^{m}$; note that the definition is independent of the branch of the square root $\nu_{z}^{-\frac{1}{2}}$. This notion is straightforwardly generalized to a function on the double cover $H_{\delta}^{\prime}: \mathcal{V}_{\left[\Omega_{\delta}, 0\right]}^{c m} \rightarrow \mathbb{C}$ and the boundary data $q: \partial \mathcal{V}_{\left[\Omega_{\delta}, 0\right]}^{m} \rightarrow \mathbb{C}$ by adding the assumption that both have monodromy -1 around the origin.

Before proving a useful uniqueness result for the discrete Riemann boundary value problems, we introduce the crucial notion of integration of the square of an s-holomorphic function, defined on the vertices and faces (see also [Smi10,Hon10, $\mathrm{CHI15]).} \mathrm{Although} \mathrm{the} \mathrm{square} \mathrm{of} \mathrm{an} \mathrm{s-holomorphic} \mathrm{function} \mathrm{is} \mathrm{not} \mathrm{s-holomorphic,} \mathrm{we}$ can "line-integrate" the square of its magnitude to obtain a single-valued function without monodromy. Its restrictions to the two rotated square lattices respectively of faces and vertices are not harmonic, but they are respectively super-harmonic and sub-harmonic, which will allow us to derive estimates crucial for proofs of the convergence.

$\mathbb{I}_{\delta}\left(H_{\delta}\right)$ is a discrete analogue of the line integral $\operatorname{Re} \int\left[H_{\delta}\right]^{2} d z$, defined as follows. 
Proposition 2.10 ([Smi10, Lemma 3.8]). Let $H_{\delta}$ be an $s$-holomorphic function on $\Omega_{\delta}$. There exists a function $\mathbb{I}_{\delta}\left[H_{\delta}\right]: \mathcal{F}_{\Omega_{\delta}} \cup \mathcal{V}_{\Omega_{\delta}} \rightarrow \mathbb{R}$ uniquely constructed (up to an additive constant) with the rule

$$
\mathbb{I}_{\delta}\left[H_{\delta}\right](w)-\mathbb{I}_{\delta}\left[H_{\delta}\right](v)=2 \delta\left|H_{\delta}\left(\frac{1}{2}(w+v)\right)\right|^{2},
$$

where $w$ is a face, $v$ is a vertex incident to the face, so that $\frac{1}{2}(w+v)$ is the corner between them.

It has $\Delta_{\delta} \mathbb{I}_{\delta}\left[H_{\delta}\right]=2 \delta\left|\partial_{\delta} H_{\delta}\right|^{2}$ on $\mathcal{F}_{\Omega_{\delta}}, \Delta_{\delta} \mathbb{I}_{\delta}\left[H_{\delta}\right]=-2 \delta\left|\partial_{\delta} H_{\delta}\right|^{2}$ on $\mathcal{V}_{\Omega_{\delta}}$.

The following uniqueness statement for both types of the discrete Riemann boundary value problems then allows one to characterize s-holomorphic functions in terms of their boundary values.

Lemma 2.11. If $H_{\delta}$ is a solution of the discrete Riemann boundary value problem on $\Omega_{\delta}$ with boundary data 0 , it is identically zero. Similarly, if $H_{\delta}^{\prime}$ is a solution of the discrete Riemann boundary value problem on $\left[\Omega_{\delta}, 0\right]$ with boundary data 0 , it is identically zero.

Proof. The case of $H_{\delta}$ is treated in [Hon10, Proposition 28], but we summarize it here. Given s-holomorphicity and $\mathrm{P}_{\nu_{z}^{-\frac{1}{2}}} H_{\delta}=0$, we can calculate $\partial_{\nu_{z}} \mathbb{I}_{\delta}\left(H_{\delta}\right)(z)=$ $\sqrt{2} \delta\left|\mathrm{P}_{i \nu_{z}^{-\frac{1}{2}}} H_{\delta}(z)\right|^{2}$. Then by using the discrete divergence formula ([Hon10, Lemma 6]) and the Laplacian, we can bound from above the orthogonal component of $H_{\delta}$ on the boundary:

$$
0 \leq \sum_{z \in \partial \mathcal{V}_{\Omega_{\delta}}^{m}} \partial_{\nu_{z}} \mathbb{I}_{\delta}\left(H_{\delta}\right)(z)=\sum_{v \in \mathcal{V}_{\Omega_{\delta}}} \Delta_{\delta} \mathbb{I}_{\delta}\left(H_{\delta}\right)(v) \leq 0
$$

which implies that $H_{\delta} \equiv 0$ on $\partial \mathcal{V}_{\Omega_{\delta}}^{m}$ and that $\Delta_{\delta} \mathbb{I}_{\delta}\left[H_{\delta}\right]=-2 \delta\left|\partial_{\delta} H_{\delta}\right|^{2} \equiv 0$ in $\mathcal{V}_{\Omega_{\delta}}$, so $H_{\delta} \equiv 0$ in $\mathcal{V}_{\Omega_{\delta}}$.

For $H_{\delta}^{\prime}$, note that (see [ChIz13, Proposition 4.1]) we can similarly define the singlevalued square integral $\mathbb{I}_{\delta}\left(H_{\delta}^{\prime}\right)$ with single valued increments $\mathbb{I}_{\delta}\left[H_{\delta}^{\prime}\right](w)-\mathbb{I}_{\delta}\left[H_{\delta}^{\prime}\right](v)=$ $2 \delta\left|H_{\delta}^{\prime}\left(\frac{1}{2}(w+v)\right)\right|^{2}$. While its restriction to faces fails to be sub-harmonic at the monodromy face in general, $\mathbb{I}_{\delta}\left(H_{\delta}^{\prime}\right)$ on vertices is nonetheless super-harmonic everywhere with positive outer difference, and we can apply the same argument as in the $H_{\delta}$ case.

\section{Discrete Two-Point Observables}

In this section, we introduce discrete observables, which connect Ising model correlations to discrete complex analysis. Bounded domain observables are defined by summing Boltzmann weights over the set of contours made of the edges in the lattice, alluding to a path integral formulation.

In this section, we define the two-point functions, in terms of which the correlations will be formulated in Propositions 4.16 and 4.17. In Sect. 4, multipoint versions of these observables are introduced to prove these statements. 
3.0.1. Low-temperature expansion In this paper, we will use the low-temperature expansion of the Ising model: we represent a spin configuration by the set of edges separating faces of opposite spins. Through this representation, the probability of a set of edges $\omega$ is proportional to $e^{-2 \beta|\omega|}$. When considering + boundary conditions, the relevant set of edges form a collection of loops (sets of distinct edges $\left\{e_{1}, \ldots, e_{k}\right\}$ such that $e_{i}$ is incident to $e_{i+1}$ for every $1 \leq i \leq k$ with $e_{k+1}=e_{1}$ ). In identifying an edge configuration $\omega$ in which every vertex is incident to an even number of edges, with a collection of loops, there is a possible ambiguity at vertices incident to four edges. For concreteness, we fix the convention that at such ambiguous vertices, loops proceed by joining northwest edges to northeast edges and southwest edges to southeast edges. We further prove that all quantities we consider are independent of choice of convention at ambiguous vertices. Denote by $\mathcal{C}_{\Omega_{\delta}}$ the set of all such $\omega$ (subsets of edges of $\mathcal{E}_{\Omega_{\delta}}$ with every vertex incident an even number of edges), corresponding to collections of closed loops in $\Omega_{\delta}$.

As a result, for the critical Ising model with + boundary conditions, the lowtemperature expansion of the partition function is thus obtained by summing over the set $\mathcal{C}_{\Omega_{\delta}}$ :

$$
\mathcal{Z}_{\Omega_{\delta}}:=\sum_{\omega \in \mathcal{C}_{\Omega_{\delta}}} e^{-2 \beta_{c}|\omega|}
$$

We also note that in this representation, the value of a spin is determined by the parity of the number of loops around it (independently of the choice of convention above), and it is easy to see that

$$
\mathbb{E}_{\Omega_{\delta}}\left[\sigma_{0}\right]=\frac{\sum_{\omega \in \mathcal{C}_{\Omega_{\delta}}} e^{-2 \beta_{c}|\omega|}(-1)^{\ell(\omega)}}{\mathcal{Z}_{\Omega_{\delta}}},
$$

where $\ell(\omega)$ counts the number of loops in $\omega$ that surround 0 .

3.0.2. Disorder lines The main tool in the study of the 2D Ising model is its fermionic formulation. In this paper, we use the low-temperature representation of the Ising fermion. The relevant sets of contours are deformed versions of $\mathcal{C}_{\Omega_{\delta}}$ above: in addition to the collection of closed loops in $\Omega_{\delta}$, there are paths linking a pair of marked points of the lattice. In the language of Kadanoff and Ceva (see [KaCe71]), these correspond to the results of the insertion of disorder operators next to the spin.

A medial vertex divides the corresponding edge into two half-edges. A walk between two medial vertices $a, z$ is a sequence that consists of a half-edge of $a$, then continues on successively adjacent, all distinct edges, before reaching a half-edge of $z$. If $\gamma^{a, z}$ is any such a walk, $\mathcal{C}_{\Omega_{\delta}}^{a, z}:=\left\{\omega \oplus \gamma^{a, z}: \omega \in \mathcal{C}_{\Omega_{\delta}}\right\}$, where $\oplus$ denotes the symmetric difference operation (where the symmetric difference of a half-edge and its edge is defined in the natural way), clearly does not depend on the choice of $\gamma^{a, z}$. Each $\gamma \in \mathcal{C}_{\Omega_{\delta}}^{a, z}$ corresponds to a walk from $a$ and to $z$ and possibly some collection of loops.

For an element $\gamma \in \mathcal{C}_{\Omega_{\delta}}^{a, z}$, we say a walk $\pi(\gamma) \subset \gamma$ from $a$ to $z$ is an admissible choice of walk if whenever it arrives at an ambiguous vertex, i.e. incident to four edges in $\gamma$, it chooses to connect northeast with northwest edges and southeast with southwest edges (in accordance with the aforementioned convention for loops). Again, we will prove well-definedness of relevant quantities so that the choice of convention here is irrelevant. When one or both of $a, z$ are instead corners, the above is defined analogously, where "half-edge" is understood to mean the segment joining the corner to its nearest vertex. 


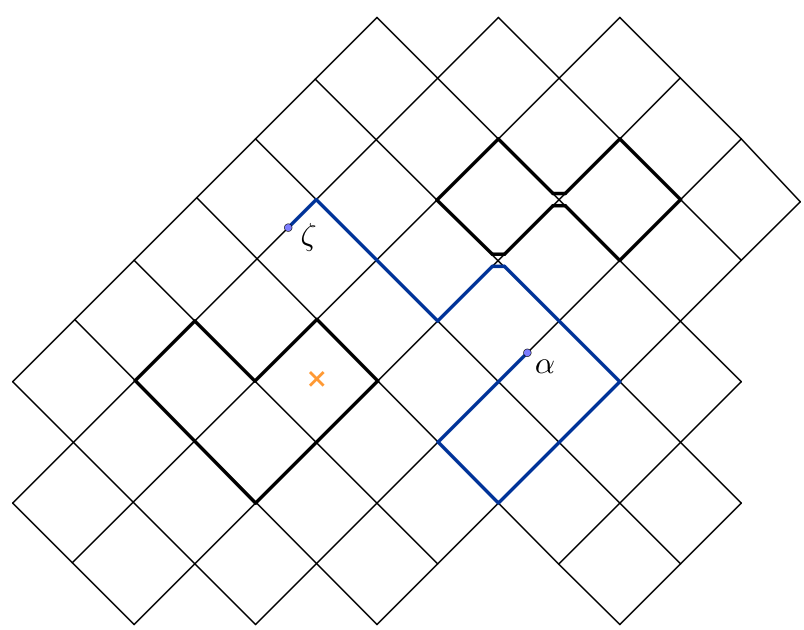

Fig. 3. An configuration $\gamma \in \mathcal{C}_{\left[\Omega_{\delta}, 0\right]}^{\alpha, \zeta}$, with an admissible choice of walk $\pi(\gamma)$ in blue, from $\alpha$ to $\zeta$. The winding of the walk has $\mathbf{W}(\pi(\gamma))=2 \pi$. The loop number $\ell(\gamma)$ is 1 since there is precisely one loop with 0 in its interior and $s_{\alpha, \zeta}(\pi(\gamma))=1$

Recall that any choice of orientation on a medial vertex is in the direction of exactly one of its two incident half-edges (see Sect.1.5). If $\alpha=a^{o}$ and $\zeta=z^{p}$ are oriented medial vertices, set $\mathcal{C}_{\Omega_{\delta}}^{\alpha, \zeta}$ to be the subset of $\gamma \in \mathcal{C}_{\Omega_{\delta}}^{a, z}$ including the particular half-edges given by the respective orientations at $a$ and $z$.

3.1. Bounded domain observables. In this subsection, we define the fermion and the spin-fermion observables. The former is a function defined on the discrete domain $\Omega_{\delta}$, whereas the latter is defined on the double cover, $\left[\Omega_{\delta}, 0\right]$. Using the above definitions of loops, walks, $\mathcal{C}_{\Omega_{\delta}}^{\alpha, \zeta}$, and admissible choices of walks, we define some quantities central to the presentation of the (two-point) fermion and spin-fermion observables (see Fig. 3).

Definition 3.1. If $\alpha=a^{o}$ and $\zeta=z^{p}$ are s-oriented medial vertices or corners, define the constants,

$$
\mathrm{c}_{v}:=\left\{\begin{array}{ll}
1 & \text { if } v \in \mathcal{V}_{\Omega_{\delta}}^{m} \\
\cos \frac{\pi}{8} & \text { if } v \in \mathcal{V}_{\Omega_{\delta}}^{c}
\end{array} \quad \text { and } \quad \lambda_{\alpha, \zeta}:=\frac{\sqrt{p}}{\sqrt{o}} \mathrm{c}_{a} \mathrm{c}_{z} .\right.
$$

For a walk and loops $\gamma \in \mathcal{C}_{\Omega_{\delta}}^{\alpha, \zeta}$, define its length $|\gamma|$ as the number of full edges (where the two half-edges at the ends together count as one) in $\gamma$ and for an admissible choice of walk $\pi(\gamma)$ in $\gamma$ from $a$ to $z$, denote its winding (more accurately, turning) angle by $\mathbf{W}(\pi(\gamma))$ as the total change in argument of the velocity vector of the walk $\pi(\gamma)$ from $a$ to $z$ (see $\$ 5.2 .1$ of [Hon10]). The choice of counting two half-edges together as one full-edge, is different from the counting in [CHI15]; this leads to an appearance of a normalizing factor of $\left(\cos \frac{\pi}{8}\right)^{2} e^{-2 \beta_{c}}=\frac{1}{2 \sqrt{2}}$, whenever considering observables with both arguments in corners.

The following is a real-valued weight on $\gamma \in \mathcal{C}_{\Omega_{\delta}}^{\alpha, \zeta}$ :

$$
\phi_{\alpha, \zeta}(\gamma):=i \lambda_{\alpha, \zeta} e^{-2 \beta_{c}|\gamma|} e^{-\frac{i}{2} \mathbf{W}(\pi(\gamma))} .
$$


We also recall for a collection of loops $\omega \in \mathcal{C}_{\Omega_{\delta}}$ the definition of $\ell(\omega)$ as the number of loops of $\omega$ around 0 (whose parity is independent of our convention for loops). See [Hon10, Proposition 67] for the well-definedness (i.e., independence of the choice of convention for the admissible path $\pi(\gamma))$ of $\phi_{\alpha, \zeta}$.

When $\alpha, \zeta$ are s-oriented medial vertices or corners on $\left[\Omega_{\delta}, 0\right]$, we define the spinfermion weight as

$$
\phi_{\alpha, \zeta}^{\Sigma}(\gamma):=\phi_{\alpha, \zeta}(\gamma)(-1)^{\ell(\gamma \backslash \pi(\gamma))} \mathrm{s}_{\alpha, \zeta}(\pi(\gamma)),
$$

where $\pi(\gamma)$ is any admissible choice of walk, and $\mathrm{s}_{\alpha, \zeta}$ is the sheet number defined by

$$
\mathrm{s}_{\alpha, \zeta}(\pi(\gamma))=\left\{\begin{array}{ll}
+1 & \text { if } \pi(\gamma) \text { lifted to }\left[\Omega_{\delta}, 0\right] \text { connects } \alpha \text { to } \zeta \\
-1 & \text { if } \pi(\gamma) \text { lifted to }\left[\Omega_{\delta}, 0\right] \text { connects } \alpha \text { to } \zeta^{*}
\end{array},\right.
$$

where $\zeta^{*}$ is the point on $\left[\Omega_{\delta}, 0\right]$ that is distinct from $\zeta$ but shares its projection with $\zeta$. Here, the real-valued weight $\phi_{\alpha, \zeta}(\gamma)$ is still computed by identifying $\alpha, \zeta$ with their projections to $\Omega_{\delta}$. $\phi_{\alpha, \zeta}^{\Sigma}$

See Remark 2.2(ii) of [CHI15] for the well-definedness of the spin-fermion weight

We are now in position to define the real fermion $F_{\Omega_{\delta}}^{\alpha, \zeta}$ and the real spin-fermion $F_{\left[\Omega_{\delta}, 0\right]}^{\alpha, \zeta}$.

Definition 3.2. The (real) fermion observable $F_{\Omega_{\delta}}$ is a function of two variables $(\alpha, \zeta) \mapsto F_{\Omega_{\delta}}^{\alpha, \zeta}$, where $\alpha:=a^{o}$ and $\zeta:=z^{p}$ are s-oriented corners or medial vertices of $\Omega_{\delta}$ given by

$$
F_{\Omega_{\delta}}^{\alpha, \zeta}:=\frac{1}{\mathcal{Z}_{\Omega_{\delta}}} \sum_{\gamma \in \mathcal{C}_{\Omega_{\delta}, \zeta}} \phi_{\alpha, \zeta}(\gamma)
$$

Definition 3.3. The (real) spin-fermion observable $F_{\left[\Omega_{\delta}, 0\right]}$ is a function of two variables $(\alpha, \zeta) \mapsto F_{\left[\Omega_{\delta}, 0\right]}^{\alpha, \zeta}$, where $\alpha:=a^{o}$ and $\zeta:=z^{p}$ live on the double cover $\left[\Omega_{\delta}, 0\right]$ of domain $\Omega_{\delta}$ ramified at 0 . Define $F_{\left[\Omega_{\delta}, 0\right]}^{\alpha, \zeta}$ by

$$
F_{\left[\Omega_{\delta}, 0\right]}^{\alpha, \zeta}:=\frac{1}{\mathcal{Z}_{\Omega_{\delta}} \mathbb{E}_{\Omega_{\delta}}\left[\sigma_{0}\right]} \sum_{\gamma \in \mathcal{C}_{\Omega_{\delta}, \zeta}^{\alpha, \zeta}} \phi_{\alpha}^{\Sigma}(\gamma)
$$

Again, when computing $\mathcal{Z}_{\Omega_{\delta}}$, and $\mathcal{C}_{\Omega_{\delta}}^{\alpha, \zeta}$ and $\phi_{\alpha, \zeta}$, identify $\alpha, \zeta$ with their projections to $\Omega_{\delta}$.

The well-definedness of $F_{\Omega_{\delta}}^{\alpha, \zeta}$ and $F_{\left[\Omega_{\delta}, 0\right]}^{\alpha, \zeta}$ are implied by well-definedness of $\phi_{\alpha, \zeta}$ and $\phi_{\alpha, \zeta}^{\Sigma}$ respectively.

Remark 3.4. Informally, one can think of $F_{\left[\Omega_{\delta}, 0\right]}$ as the natural modification to $F_{\Omega_{\delta}}$ when one tries to reweight it by the value of the spin at 0 . Since the spin and the disorders are not mutually local (but quasi-local instead), this gives rise to a multivalued function (with monodromy -1 around 0 ). 
3.1.1. Antisymmetry of the observables An important elementary feature of the observables $F_{\Omega_{\delta}}, F_{\left[\Omega_{\delta}, 0\right]}$ is their antisymmetry properties, immediate from their definitions. The fermion observable satisfies the following:

Lemma 3.5. If $\alpha:=a^{o}, \zeta:=z^{p}$ are s-oriented corners or medial vertices of $\Omega_{\delta}$ and $\alpha^{\prime}:=a^{o^{\prime}}, \zeta^{\prime}:=z^{p^{\prime}}$ where $o^{\prime}:=e^{2 \pi i} o$ and $p^{\prime}:=e^{2 \pi i} p$, then we have the following antisymmetry properties:

$$
F_{\Omega_{\delta}}^{\alpha, \zeta}=-F_{\Omega_{\delta}}^{\zeta, \alpha}=-F_{\Omega_{\delta}}^{\alpha^{\prime}, \zeta}=-F_{\Omega_{\delta}}^{\alpha, \zeta^{\prime}} .
$$

Similarly, the spin-fermion observable satisfies the following antisymmetry properties.

Lemma 3.6. If $\alpha:=a^{o}, \zeta:=z^{p}$ are s-oriented corners or medial vertices of $\left[\Omega_{\delta}, 0\right]$, and $\alpha^{\prime}, \zeta^{\prime}$ are as in the previous lemma and $\alpha^{*}:=\left(a^{*}\right)^{o}, \zeta^{*}:=\left(z^{*}\right)^{p}$ where $a, a^{*}$ and $z, z^{*}$ are respectively distinct lifts of the same points in $\Omega \backslash\{0\}$, we have

$$
F_{\left[\Omega_{\delta}, 0\right]}^{\alpha, \zeta}=-F_{\left[\Omega_{\delta}, 0\right]}^{\zeta, \alpha}=-F_{\left[\Omega_{\delta}, 0\right]}^{\alpha^{\prime}, \zeta}=-F_{\left[\Omega_{\delta}, 0\right]}^{\alpha, \zeta^{\prime}}=-F_{\left[\Omega_{\delta}, 0\right]}^{\alpha^{*}, \zeta}=-F_{\left[\Omega_{\delta}, 0\right]}^{\alpha, \zeta^{*}} .
$$

Recall that we define complex conjugation on the double cover by letting the square root be conjugated. We similarly conjugate the s-orientations, and define $\bar{\alpha}:=\bar{a}^{\bar{o}}$ if $\alpha=a^{o}$. The fact that the contour set of $\Omega_{\delta}$ and its mirror image $\overline{\Omega_{\delta}}$ have a natural bijection arising from the complex conjugation immediately yields the following.

Lemma 3.7. If $\alpha, \zeta$ are s-oriented corners or medial vertices in $\left[\Omega_{\delta}, 0\right]$, we have $F_{\left[\Omega_{\delta}, 0\right]}^{\alpha, \zeta}=-F_{\left[\overline{\Omega_{\delta}}, 0\right]}^{\bar{\alpha}, \bar{\zeta}}$

3.1.2. Complexified observables As explained in the previous subsections, the observables introduced in the previous subsections are real quantities antisymmetric in their two variables; exploiting those properties we can define the following complexified versions, which can be analyzed with discrete complex analysis.

Definition 3.8. Let $\alpha$ be an s-oriented corner or medial vertex of $\Omega_{\delta}$. For an (unoriented) medial vertex $z \in \mathcal{V}_{\Omega_{\delta}}^{m}$, we define the complex fermion-fermion observable $H_{\Omega_{\delta}}^{\alpha}$ by

$$
H_{\Omega_{\delta}}^{\alpha}(z):=\frac{1}{i \sqrt{p_{1}}} F_{\Omega_{\delta}}^{\alpha, \zeta^{1}}+\frac{1}{i \sqrt{p_{2}}} F_{\Omega_{\delta}}^{\alpha, \zeta^{2}},
$$

where $\zeta^{1}:=z^{p_{1}}$ and $\zeta^{2}:=z^{p_{2}}$ are arbitrary s-orientations of $z$ with opposite orientations, i.e. $p_{2}=e^{ \pm \pi i} p_{1}$. The resulting quantity is easily seen to be well-defined regardless of the choice of s-orientations. Similarly, for a corner $c \in \mathcal{V}_{\Omega_{\delta}}^{c}$ with s-orientation $\kappa=c^{q}$, define

$$
H_{\Omega_{\delta}}^{\alpha}(c):=\frac{1}{i \sqrt{q}} F_{\Omega_{\delta}}^{\alpha, \kappa} .
$$

Define the complexified spin-fermion observable in the same way:

Definition 3.9. Let $\alpha$ be an s-oriented corner or medial vertex of $\left[\Omega_{\delta}, 0\right]$, let $z$ be a medial vertex of $\left[\Omega_{\delta}, 0\right]$, and let $c$ be a corner of $\left[\Omega_{\delta}, 0\right]$. Using the same notation as in Definition 3.8, we define the complex spin-fermion observable $H_{\left[\Omega_{\delta}, 0\right]}$ by

$$
\begin{aligned}
H_{\left[\Omega_{\delta}, 0\right]}^{\alpha}(z) & :=\frac{1}{i \sqrt{p_{1}}} F_{\left[\Omega_{\delta}, 0\right]}^{\alpha, \zeta^{1}}+\frac{1}{i \sqrt{p_{2}}} F_{\left[\Omega_{\delta}, 0\right]}^{\alpha, \zeta^{2}}, \\
H_{\left[\Omega_{\delta}, 0\right]}^{\alpha}(c) & :=\frac{1}{i \sqrt{q}} F_{\left[\Omega_{\delta}, 0\right]}^{\alpha, \kappa} .
\end{aligned}
$$


Remark 3.10. Note that, given a complexified observable, the real observables can be recovered by $F_{\Omega_{\delta}}^{\alpha, \zeta}=i \sqrt{p} \mathrm{P} \frac{1}{i \sqrt{p}} \mathbb{R}\left[H_{\Omega_{\delta}}^{\alpha}(z)\right]=\operatorname{Re}\left[i \sqrt{p} H_{\Omega_{\delta}}^{\alpha}(z)\right]$ for medial vertices $\zeta=z^{p}$, and obviously at corners $\kappa=c^{q}$, we have $F_{\Omega_{\delta}}^{\alpha, \kappa}=\operatorname{Re}\left[i \sqrt{q} H_{\Omega_{\delta}}^{\alpha}(c)\right]$.

Definition 3.11. Let $\Lambda_{\delta}=\Omega_{\delta} \cap \overline{\Omega_{\delta}}$. Then define the symmetrized and antisymmetrized observables:

$$
S_{\left[\Omega_{\delta}, 0\right]}^{\alpha}:=\frac{1}{2}\left[H_{\left[\Omega_{\delta}, 0\right]}^{\alpha}+H_{\overline{\left[\Omega_{\delta}, 0\right]}}^{\bar{\alpha}}\right], \quad A_{\left[\Omega_{\delta}, 0\right]}^{\alpha}:=\frac{1}{2}\left[H_{\left[\Omega_{\delta}, 0\right]}^{\alpha}-H_{\left[\Omega_{\delta}, 0\right]}^{\bar{\alpha}}\right] .
$$

The following lemma then follows immediately from Lemma 3.5 and the definition of $H_{\left[\Omega_{\delta}, 0\right]}$.

Lemma 3.12. Let $\alpha$ be an s-oriented corner or medial vertex in $\left[\Omega_{\delta}, 0\right]$, and $z$ be a corner or medial vertex in $\left[\Lambda_{\delta}, 0\right]$. Then,

$$
\begin{array}{ll}
\text { on } \mathcal{V}_{\left[\Omega_{\delta}, 0\right]}^{1} \cap \mathbb{R}_{>0}, \mathcal{V}_{\left[\Omega_{\delta}, 0\right]}^{i} \cap \mathbb{R}_{<0}, \quad \text { we have } & S_{\left[\Omega_{\delta}, 0\right]}^{\alpha}=H_{\left[\Omega_{\delta}, 0\right]}^{\alpha} \text { and } A_{\left[\Omega_{\delta}, 0\right]}^{\alpha}=0 ; \\
\text { on } \mathcal{V}_{\left[\Omega_{\delta}, 0\right]}^{i} \cap \mathbb{R}_{>0}, \mathcal{V}_{\left[\Omega_{\delta}, 0\right]}^{1} \cap \mathbb{R}_{<0}, \quad \text { we have } & A_{\left[\Omega_{\delta}, 0\right]}^{\alpha}=H_{\left[\Omega_{\delta}, 0\right]}^{\alpha} \text { and } S_{\left[\Omega_{\delta}, 0\right]}^{\alpha}=0 .
\end{array}
$$

3.2. Full plane observables. In this section, we study the infinite-volume limits of the fermion and spin-fermion observables. By scale invariance, it is enough to give a characterization on the rotated unit grid $\mathbb{C}_{1}=(1+i) \mathbb{Z}^{2}+1$ placed on increasing domains. On $\mathbb{C}_{\delta}$ we can define $H_{\mathbb{C}_{\delta}}(a \delta):=H_{\mathbb{C}_{1}}(a)$ and $H_{\left[\mathbb{C}_{\delta}, 0\right]}(a \delta):=H_{\left[\mathbb{C}_{1}, 0\right]}(a)$. In the $\delta \rightarrow 0$ scaling limit, these converge to meromorphic functions with a singularity at zero.

We first give a unique characterization for the full-plane limits and establish their existence, and then we give an explicit construction. Using those explicit formulae, we define auxiliary s-holomorphic functions on the double cover which are discrete forms of $\sqrt{z}$ and $i \sqrt{z}$.

We take the limit $\Omega_{1} \rightarrow \mathbb{C}_{1}$ using an increasing sequence of bounded domains $\Omega_{1}^{1} \subset \Omega_{1}^{2} \subset \cdots \subset \Omega_{1}^{n} \subset \cdots$ such that $\bigcup_{n} \Omega_{1}^{n}=\mathbb{C}_{1}$. The limiting functions will be seen to be unique, so that they do not depend on the particular sequence.

3.2.1. Full plane fermion observable The following are straightforward modifications to our setting, of the construction [Hon10] of the full-plane fermion.

Theorem 3.13. As $\Omega_{1} \rightarrow \mathbb{C}_{1}$, the complexified fermion observable $H_{\mathbb{C}_{1}}:=\lim _{\Omega \rightarrow \mathbb{C}} H_{\Omega_{1}}$ exists and is uniquely characterized by the following properties:

- if $\alpha=a^{o}$ is an s-oriented medial vertex,

- $H_{\mathbb{C}_{1}}^{\alpha}$ is s-holomorphic on $\mathbb{C}_{1} \backslash\{a\}$;

- At $\alpha$ we have the discrete residue $H_{\mathbb{C}_{1}}^{\alpha}\left(\alpha_{+}\right)-H_{\mathbb{C}_{1}}^{\alpha}\left(\alpha_{-}\right)=\frac{1}{\sqrt{o}}$;

- $H_{\mathbb{C}_{1}}^{\alpha}(l) \rightarrow 0$ as $|l| \rightarrow \infty$.

- For $\zeta=z^{p}$, the full-plane fermion

$$
F_{\mathbb{C}_{1}}^{\alpha, \zeta}:=i \sqrt{p} \cdot \mathrm{P}_{\frac{1}{i \sqrt{p}}} \mathbb{R}\left[H_{\mathbb{C}_{1}}^{\alpha}(z)\right]
$$

satisfies the antisymmetry properties of Lemma 3.5. 
Proof. For a given s-oriented medial vertex $\alpha$, such an $H_{\mathbb{C}_{1}}^{\alpha}$ must be uniquely determined: if any two satisfy the above properties, their difference will have an s-holomorphic extension to $a$, but any entire s-holomorphic function which decays to 0 at infinity must be zero, since its real and imaginary parts will be discrete harmonic functions.

To use the same reasoning when $\alpha$ is an s-oriented corner with adjacent medial vertices $z, z^{\prime}$, it suffices to show that the s-holomorphic singularity at $\alpha$, i.e. concretely the value of nonzero $\mathrm{P}_{l(\alpha)}\left[H_{\mathbb{C}_{1}}^{\alpha}\left(z^{\prime}\right)\right]-\mathrm{P}_{l(\alpha)}\left[H_{\mathbb{C}_{1}}^{\alpha}(z)\right]$, is fixed by the medial vertex case above. But the antisymmetry relation of Lemma 3.5 gives $H_{\mathbb{C}_{1}}^{\alpha}(z)=\frac{1}{i \sqrt{p_{1}}} F_{\mathbb{C}_{1}}^{\alpha, \zeta^{1}}+\frac{1}{i \sqrt{p_{2}}} F_{\mathbb{C}_{1}}^{\alpha, \zeta^{2}}$ for $\zeta^{1}=z^{p_{1}}$ and $\zeta^{2}=z^{p_{2}}$, where $p_{1}$ and $p_{2}$ are s-orientations of the two opposite orientations of $z$. Since $F_{\mathbb{C}_{1}}^{\alpha, \zeta^{1}}=-F_{\mathbb{C}_{1}}^{\zeta^{1}, a}$ and $F_{\mathbb{C}_{1}, \zeta^{2}}=-F_{\mathbb{C}_{1}, \zeta^{2}, a}$, both terms are determined by their values on medial vertices, and similarly for $H_{\mathbb{C}_{1}}^{\alpha}\left(z^{\prime}\right)$.

An explicit formula, Eq. (3.1), for this full-plane observable, and thus its existence, is given by Proposition 3.14. Then the fact that the given explicit function is the infinitevolume limit is immediate from Theorem 5.11.

Proposition 3.14. Let $a, z \in \mathcal{V}_{\mathbb{C}_{1}}^{m}$ and for an s-orientation $(\sqrt{o})^{2}$ on $a$, write $\alpha=a^{o}$ for the s-oriented medial vertex. The function

$$
\begin{aligned}
H_{\mathbb{C}_{1}}^{\alpha}(z)= & \frac{e^{\pi i / 8}}{\sqrt{o}} \cos \frac{\pi}{8}\left(C_{0}\left(\frac{\sqrt{2} a}{o}+1, \frac{\sqrt{2} z}{o}\right)+C_{0}\left(\frac{\sqrt{2} a}{o}-i, \frac{\sqrt{2} z}{o}\right)\right) \\
& +\frac{e^{-3 \pi i / 8}}{\sqrt{o}} \sin \frac{\pi}{8}\left(C_{0}\left(\frac{\sqrt{2} a}{o}-1, \frac{\sqrt{2} z}{o}\right)+C_{0}\left(\frac{\sqrt{2} a}{o}+i, \frac{\sqrt{2} z}{o}\right)\right)
\end{aligned}
$$

for $z \neq a$ satisfies the properties of Theorem 3.13, where the translation invariant function $C_{0}$ is the dimer coupling function defined in [Ken00]:

$$
C_{0}\left(z_{1}, z_{2}\right)=\frac{1}{4 \pi^{2}} \int_{0}^{2 \pi} \int_{0}^{2 \pi} \frac{\exp (i(x s-y t))}{2 i \sin s+2 \sin t} d s d t, \quad \text { if } z_{2}-z_{1}=x+i y .
$$

Moreover, at $\alpha$, the front and back values of the s-holomorphic singularity are given by $H_{\mathbb{C}_{1}}^{\alpha}\left(\alpha_{ \pm}\right)=\frac{\mu \pm 1}{2 \sqrt{o}}$.

Proof. These properties were verified in [Hon10, Proposition 22] for a version on the non-rotated lattice, which we will call $H_{\mathbb{Z}^{2}}$. We note that for any s-oriented medial vertex $\alpha=a^{o}$, we have

$$
H_{\mathbb{C}_{1}}^{\alpha}(z)=e^{-\frac{\pi i}{8}} H_{\mathbb{Z}^{2}}^{\alpha^{\prime}}\left(z^{\prime}\right)
$$

if $\alpha^{\prime}=\left(a^{\prime}\right)^{o^{\prime}}$ is the rotated medial vertex $a^{\prime}=\frac{a}{1+i} \in \mathcal{V}_{\mathbb{Z}^{2}}^{m}$ oriented to $\sqrt{o^{\prime}}=e^{-\frac{\pi i}{8}} \sqrt{o}$, and $z^{\prime}=\frac{z}{1+i}$. Given that the projection lines in the s-holomorphicity relation Eq. (2.1) are also rotated by $e^{-\frac{\pi i}{8}}$ from the definition in [Hon10], the results are easily seen to carry over. The explicit front and back values $H_{\mathbb{C}_{1}}^{\alpha}\left(\alpha_{ \pm}\right)$follow from straightforward computation. 
3.2.2. Full plane spin-fermion observable First we recall some notation. Define the left slit plane $\mathbb{X}=\mathbb{C} \backslash \mathbb{R}_{<0}$ and the right slit plane $\mathbb{Y}=\mathbb{C} \backslash \mathbb{R}_{>0}$. The double cover $[\mathbb{C}, 0]$ contains two lifts $\mathbb{X}^{ \pm}$of $\mathbb{X}$ and two lifts $\mathbb{Y}^{ \pm}$of $\mathbb{Y}$; define $\sqrt{z}$ on the double cover such that the superscripts of $\mathbb{X}^{ \pm}$denote the sign of the real part of $\sqrt{z}$ and those of $\mathbb{Y}^{ \pm}$denote the sign of the imaginary part of $\sqrt{z}$. In other words, $\mathbb{X}^{+} \cap \mathbb{Y}^{+}, \mathbb{X}^{-} \cap \mathbb{Y}^{-}$are lifts of the upper half plane, and $\mathbb{X}^{+} \cap \mathbb{Y}^{-}, \mathbb{X}^{-} \cap \mathbb{Y}^{+}$are lifts of the lower half plane. We use the process outlined in Remark 2.3 to define an s-holomorphic extension from a discrete holomorphic function defined on type 1 and $i$ corners, so let us define the slit discrete domains $\mathbb{X}_{1}^{1}:=\mathcal{V}_{\mathbb{C}_{1}}^{1} \cap \mathbb{X} \cong \mathcal{V}_{\left[\mathbb{C}_{1}, 0\right]}^{1} \cap \mathbb{X}^{ \pm}$and $\mathbb{Y}_{1}^{i}:=\mathcal{V}_{\mathbb{C}_{1}}^{i} \cap \mathbb{Y} \cong \mathcal{V}_{\left[\mathbb{C}_{1}, 0\right]}^{i} \cap \mathbb{Y}^{ \pm}$.

Theorem 3.15. As $\Omega_{1} \rightarrow \mathbb{C}_{1}$, the complexified spin-fermion observable $H_{\left[\mathbb{C}_{1}, 0\right]}:=$ $\lim _{\Omega \rightarrow \mathbb{C}} H_{\left[\Omega_{1}, 0\right]}$ exists and is uniquely characterized by the following properties: for every $\alpha=a^{o} \in \mathcal{V}_{\left[\mathbb{C}_{1}, 0\right]}^{c m}$,

A $H_{\left[\mathbb{C}_{1}, 0\right]}^{\alpha}$ has monodromy -1 around 0 .

$B H_{\left[\mathbb{C}_{1}, 0\right]}^{\alpha}$ is s-holomorphic on $\left[\mathbb{C}_{1}, 0\right] \backslash\left\{a, a^{*}\right\}$, where $a^{*}$ is the point in $\left[\mathbb{C}_{1}, 0\right]$ distinct from $a$ which shares its projection onto $\mathbb{C}_{1}$ with the projection of $a$.

$C$ if $\alpha=a^{o}$ is an s-oriented medial vertex, we have discrete residue $H_{\left[\mathbb{C}_{1}, 0\right]}^{\alpha}\left(\alpha_{+}\right)-$ $H_{\left[\mathbb{C}_{1}, 0\right]}^{\alpha}\left(\alpha_{-}\right)=\frac{1}{\sqrt{o}}$.

$D$ If $\alpha=a^{o}$ is an s-oriented real or imaginary corner, $P_{l(a)} H_{\left[\Omega_{1}, 0\right]}^{\alpha}\left(a \pm \frac{i}{2}\right)=\mp \frac{i}{2 \sqrt{2}} \sqrt{o}$. E For $\zeta=z^{p}$, the full-plane spin-fermion,

$$
F_{\left[\mathbb{C}_{1}, 0\right]}^{\alpha, \zeta}:=i \sqrt{p} \cdot \mathrm{P}_{\frac{1}{i \sqrt{p}} \mathbb{R}}\left[H_{\left[\mathbb{C}_{1}, 0\right]}^{\alpha}(z)\right]
$$

satisfies the antisymmetry properties laid out in Lemmas 3.6-3.7.

$F H_{\left[\mathbb{C}_{1}, 0\right]}^{\alpha}(l) \rightarrow 0$ as $|l| \rightarrow \infty$.

We first present the following three lemmas, then conclude the proof using them.

Lemma 3.16. There exists a uniform constant $M>0$ such that $\left|H_{\left[\Omega_{1}^{n}, 0\right]}^{\alpha}(z)\right| \leq M$ for all $n \geq 0$ and any s-oriented corner $\alpha$ and any corner $z$.

Proof. The strategy will be to progressively extend the validity of the result to more and more points of the domain. Below we will denote by $a$ and $z$ both corners and medial vertices interchangeably:

1. When $\alpha:=\alpha_{0}$ is the imaginary corner on the monodromy face 0 (specifically, the lift of $\frac{1}{2}$ to $\left.\mathbb{X}_{\delta}\right)$ and $z=a+1, H_{\left[\Omega_{1}^{n}, 0\right]}^{\alpha}(z)$ has a probabilistic interpretation as a ratio of magnetizations $\mathbb{E}_{\Omega_{1}^{n}}\left[\sigma_{2}\right] / \mathbb{E}_{\Omega_{1}^{n}}\left[\sigma_{0}\right]$, which is bounded from above by the finite-energy property of the model.

2. When $\alpha=\alpha_{0}$ and $z$ is on the boundary, we claim that $\sum_{z \in \partial \mathcal{V}_{\left[\Omega_{1}^{n}, 0\right]}^{m}}\left|H_{\left[\Omega_{1}^{n}, 0\right]}^{\alpha}(z)\right|^{2} \leq$ Cst $\cdot H_{\left[\Omega_{1}^{n}, 0\right]}^{\alpha}(a+1)$ (the right hand side of which is bounded by step 1 ). This inequality follows by considering the discrete analogue $Q_{1}:=\mathbb{I}_{\delta}\left(H_{\left[\Omega_{1}^{n}, 0\right]}^{\alpha}\right)$ of $\operatorname{Re}\left(\int\left(H^{n, \alpha_{0}}\right)^{2}\right)$ analyzed in Sect. 5.1. By Proposition 5.3, the restriction of $Q_{1}$ to the vertices is super-harmonic (except perhaps at $a+1$ ), the sum of the Laplacians is hence bounded from above by Cst $\cdot H_{\left[\Omega_{1}^{n}, 0\right]}^{\alpha}(a+1)$. At the same time the sum of the Laplacians equals the sum of the outer normal derivatives $\partial_{\nu_{z}} Q_{1}$ on the boundary 
of $\left[\Omega_{1}^{n}, 0\right]$, and these normal derivatives $\partial_{\nu_{z}} Q_{1}$ equal $\sqrt{2}\left|H_{\left[\Omega_{1}^{n}, 0\right]}^{\alpha}(z)\right|^{2}$. Hence we deduce the inequality.

3. When $\alpha=\alpha_{0}$ and $z$ (corner or medial vertex) is in the interior, we extend the bound of step 2 by the maximum principle.

4. When $\alpha$ is on the boundary and $z$ is the imaginary corner adjacent to the monodromy $\left(z=\frac{1}{2}\right)$, the bound follows from the antisymmetry of $H$ and step 2 .

5. When $\alpha$ and $z$ are on the boundary, we have that $\left|H_{\left[\Omega_{1}^{n}, 0\right]}^{\alpha}(z)\right|$ acquires a probabilistic interpretation: the winding factors out from the sum in the definition of $H$, and we sum over contours that represent the low-temperature expansion of an Ising model with $+/-$ boundary conditions switching at $a$ and $z$. As a result, it is easy to show that $\left|H_{\left[\Omega_{1}^{n}, 0\right]}^{\alpha}(z)\right|$ is the ratio $\mathbb{E}_{\Omega_{1}^{n}}^{ \pm}\left[\sigma_{0}\right] / \mathbb{E}_{\Omega_{1}^{n}}^{+}\left[\sigma_{0}\right]$, where \pm and + indicate the boundary conditions. By monotonocity of the Ising magnetization in boundary conditions, this ratio is less than one, which gives us the desired bound.

6. When $\alpha$ is on the boundary and $z$ in the interior, the result follows from steps 4 and 5 and the maximum principle.

7. When $\alpha$ is in the interior and $z$ is next to the monodromy or on the boundary, the result follows from steps 3 and 6 by antisymmetry.

8. When $\alpha$ and $z$ are in the interior, the result follows from the maximum principle.

Lemma 3.17. Any bounded function $H:=H_{\left[\mathbb{C}_{1}, 0\right]}$ that satisfies the properties $A-E$ decays at infinity.

Proof. We exploit the antisymmetry properties E, as specified in Lemmas 3.6-3.7. The idea is to symmetrize-antisymmetrize $H$ as in Definition 3.11 by writing it as $S+A$, where $S^{\alpha}=\frac{1}{2}\left(H^{\alpha}+H^{\bar{\alpha}}\right)$ and $A^{\alpha}=\frac{1}{2}\left(H^{\alpha}-H^{\bar{\alpha}}\right)$. Let us now show that $S$ and $A$ both decay at infinity.

We have that the restriction of $S$ to real corners vanishes of the positive half-line. We can make a branch cut where it vanishes and study the function on both slit-plane sheets separated by the cut. Since it is uniformly bounded and harmonic except near 0 and $a, \bar{a}$, one can use planar random walk arguments (Beurling estimate) to show that the function vanishes at infinity.

Similarly, the restriction to imaginary corners and analogous restrictions of $A$ vanish on either the positive or the negative half-line. By the same arguments as above, we can conclude the proof.

Lemma 3.18. There is at most one function satisfying the properties $A-F$.

Proof. To prove the uniqueness, it suffices that if we have two such functions, their difference is zero. Denote by $D^{\alpha}(z)$ this difference, which will be everywhere s-holomorphic and decay at infinity. However, as noted in Remark 2.6, the absence of s-holomorphic singularities does not guarantee harmonicity on the monodromy face, and some care is needed there (note that below, we abuse notation to refer to points of $[\mathbb{C}, 0]$ by their projections on $\mathbb{C}$ ).

For $\alpha_{0}=\left(\frac{1}{2}\right)^{o}, D^{\alpha_{0}}$ extends s-holomorphically to $a_{0}$ by zero by property $\mathrm{D}$, and we have that the real part of $D^{\alpha_{0}}$ is everywhere harmonic by Remark 2.6. As a result, by the maximum principle and discrete holomorphicity, the real part of $D^{\alpha_{0}}$ vanishes and $D^{\alpha_{0}} \equiv 0$. 
For an arbitrary corner $a$, by antisymmetry and the previous step, we have $D^{\alpha}\left(a_{0}\right)=$ 0 . As a result the real part of $D^{\alpha}(z)$ is harmonic. Thus, as in the previous step, $D^{\alpha}(z)$ vanishes everywhere.

Proof of Theorem 3.15. By Lemma 3.16, we have that for each s-oriented corner $\alpha$ of $\left[\mathbb{C}_{1}, 0\right]$, the sequence of harmonic functions $H_{\left[\Omega_{1}^{n}, 0\right]}^{\alpha}$ is uniformly bounded and hence by standard arguments, it admits convergent subsequences as $n \rightarrow \infty$ on any finite graph. Any limit along such subsequences satisfies properties A-E and as a result tends to 0 at infinity by Lemma 3.17. By Lemma 3.18, it is uniquely determined. This shows the convergence of the sequence itself to a limit which satisfies the conditions of the theorem, which we call $H_{\left[\mathbb{C}_{1}, 0\right]}$.

3.2.3. Analytical expressions In this subsection, we give characterizations of $H_{\left[\mathbb{C}_{1}, 0\right]}$ in a few special cases, then outline an inductive process to construct it explicitly in general.

For the observables with monodromy, we have the following characterization near 0 from [CHI15]. Recall $\alpha_{0}=a_{0}^{o}$ where $a_{0} \in \mathbb{X}^{+}$is the lift of $\frac{1}{2}$ to $\mathbb{X}^{+}$and $o=\left(e^{2 \pi i}\right)^{2}$.

Proposition 3.19. For $z \in \mathcal{V}_{\left[\mathbb{C}_{1}, 0\right]}^{1} \cup \mathcal{V}_{\left[\mathbb{C}_{1}, 0\right]}^{i} \backslash\left\{\frac{1}{2}\right\}$ we have the characterization

$$
H_{\left[\mathbb{C}_{1}, 0\right]}^{\alpha_{0}}(z)= \begin{cases} \pm \frac{1}{2 \sqrt{2}} h m_{3 / 2}^{\mathbb{X}_{1}^{1}}(z) & \text { if } z \in \mathcal{V}_{\left[\mathbb{C}_{1}, 0\right]}^{1} \cap \mathbb{X}^{ \pm} \\ \mp \frac{i}{2 \sqrt{2}} h m m_{1 / 2}^{\mathbb{Y}_{1}^{i}}(z) & \text { if } z \in \mathcal{V}_{\left[\mathbb{C}_{1}, 0\right]}^{i} \cap \mathbb{Y}^{ \pm}, \\ 0 & \text { otherwise }\end{cases}
$$

where $h m{ }_{a}^{\mathbb{D}_{\delta}}(z)$, for a discrete domain $\mathbb{D}_{\delta}$ and $a \in \mathbb{D}_{\delta} \cup \partial \mathbb{D}_{\delta}$, denotes the harmonic measure of a as seen from $z$, i.e., the probability that a simple symmetric random walk on $\mathbb{D}_{\delta}$ started at $z$ will first hit a when or before exiting $\mathbb{D}_{\delta}$.

Proof. In [CHI15, Lemma 2.14] the function defined above (without the additional normalization factor $\cos ^{2} \frac{\pi}{8} \cdot e^{-2 \beta_{c}}=\frac{1}{2 \sqrt{2}}$ ) is proved to be the only function on $\left[\mathbb{C}_{1}, 0\right]$ which decays at infinity and is s-holomorphic everywhere away from the singularity at $a_{0}=\frac{1}{2}$ given by $\mathrm{P}_{l(a)} H_{\left[\mathbb{C}_{1}, 0\right]}^{\alpha}\left(\frac{1 \pm i}{2}\right)=\mp i$. Thus we can identify it as the unique infinite-volume limit introduced in Theorem 3.15 for $\alpha=\alpha_{0}$.

Remark 3.20. The zeros in the definition reflect the fact that a slit plane harmonic measure vanishes everywhere on the slit except at the tip, e.g. $\frac{1}{2}$ in case of $\mathrm{hm} \mathbb{1}_{1 / 2}^{i}(z)$. This function is harmonic on all points of $\mathbb{Y}_{1}^{i}$, but harmonicity fails on the slit (positive real axis), the boundary of the domain.

The following explicit characterization of the discrete harmonic measure of the slit plane may be of independent interest. Using this, we provide the values of $2 \sqrt{2} H_{\left[\mathbb{C}_{1}, 0\right]}^{\alpha_{0}}(z)$ near the origin in Fig. 4.

Theorem 3.21. We have the following expression for the discrete harmonic measure:

$$
H_{0}(z):=h m{\underset{1 / 2}{i}}_{1 / 2}^{\mathbb{P}^{i}}\left(z=s+i k+\frac{1}{2}\right)=\frac{1}{2 \pi} \int_{-\pi}^{\pi} \frac{C^{|k|}(\theta)}{\sqrt{1-e^{-2 i \theta}}} e^{-i s \theta} d \theta,
$$

where $C(\theta):=\frac{\cos \theta}{1+|\sin \theta|}$. 


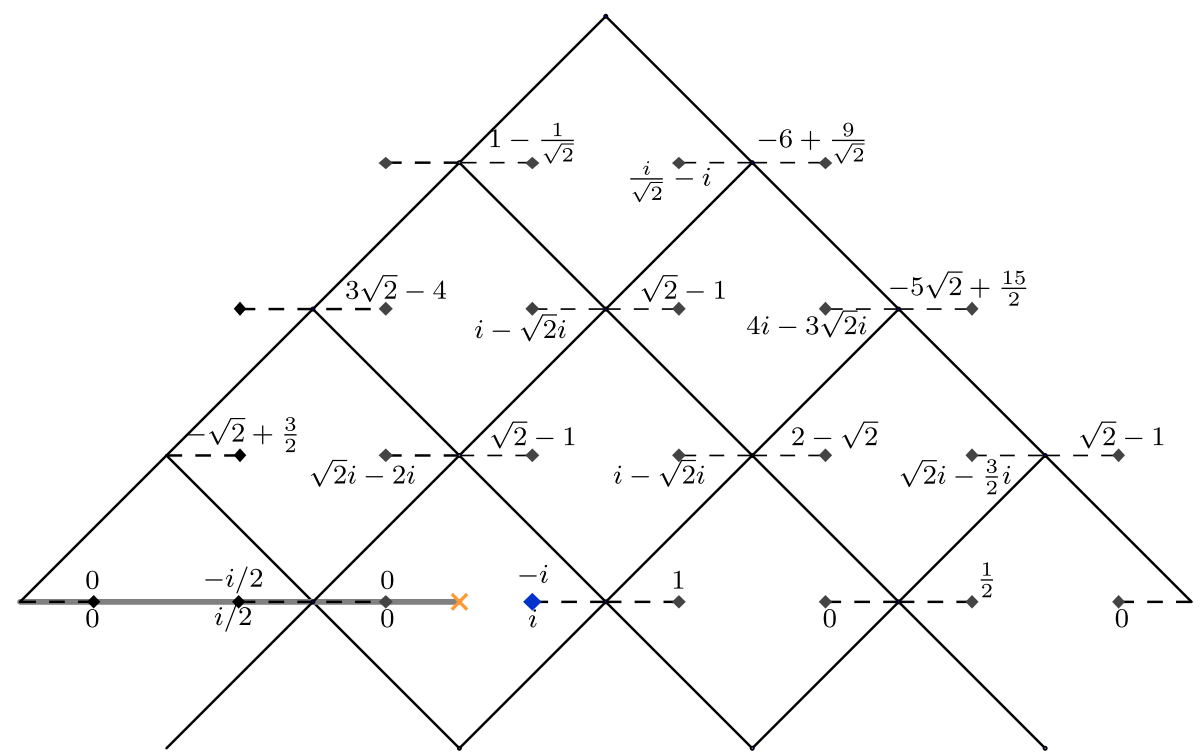

Fig. 4. Some explicit values for the full-plane spin-fermion observable $2 \sqrt{2} H_{\left[\mathbb{C}_{1}, 0\right]}^{\alpha_{0}}(z)$ for $z \in \mathcal{V}_{\left[\mathbb{C}_{1}, 0\right]}^{1, i} \cap \mathbb{X}^{+}$, where $a_{0}=\frac{1}{2}$. The function has a monodromy about the origin, marked by the orange $\times$, and singularity at $\alpha_{0}$ marked by the blue

Proof. We defer the proof of this theorem to Appendix A, Proposition A.1.

Now we inductively characterize $H_{\left[\mathbb{C}_{1}, 0\right]}^{\alpha}$ in the cases where $\alpha \in \mathcal{V}_{\left[\mathbb{C}_{1}, 0\right]}^{1, i} \cap \mathbb{R}_{>0}$.

Proposition 3.22. For an s-oriented corner $\alpha=a^{o}$ with $a \in \mathbb{X}^{+} \cap \mathbb{R}_{\geq 0}$, we can recursively compute $H_{\left[\mathbb{C}_{1}, 0\right]}^{\alpha}$ starting from the case $\alpha_{0}=\frac{1}{2}^{o_{0}}, o_{0}=\left(e^{2 \pi i}\right)^{2}$, as a finite linear combination of functions given explicitly in Theorem 3.21. Explicitly, for s-oriented corners $\alpha_{0}+2 n:=\left(a_{0}+2 n\right)^{o_{0}}, \alpha_{0}+2 n+1:=\left(a_{0}+2 n+1\right)^{o^{\prime}}, o^{\prime}=\left(e^{\frac{\pi i}{2}}\right)^{2}$, we have on any of the four half-planes $\mathbb{X}^{ \pm} \cap \mathbb{Y}^{ \pm}$,

$$
\begin{gathered}
H_{\left[\mathbb{C}_{1}, 0\right]}^{\alpha_{0}+2 n}(z)=H_{\left[\mathbb{C}_{1}, 0\right]}^{\alpha_{0}}(z-2 n)-\sum_{m=1}^{n} \frac{H_{0}(-2 m+2)}{2 m} H_{\left[\mathbb{C}_{1}, 0\right]}^{\alpha_{0}}(z-2(n-m)), \text { and } \\
H_{\left[\mathbb{C}_{1}, 0\right]}^{\alpha_{0}+2 n+1}(z)=i H_{\left[\mathbb{C}_{1}, 0\right]}^{\alpha_{0}+2 n}(z-1)-i\left(H_{0}(2 n+2)+\frac{H_{0}(2 n)}{2(n+1)}\right) H_{\left[\mathbb{C}_{1}, 0\right]}^{\alpha_{0}}(z+1) .
\end{gathered}
$$

In fact, for any $a \in \mathcal{V}_{\left[\mathbb{C}_{1}, 0\right]}^{1} \cup \mathcal{V}_{\left[\mathbb{C}_{1}, 0\right]}^{i}$ on the real or imaginary axes, we can compute $H_{\left[\mathbb{C}_{1}, 0\right]}^{\alpha}$ using rotational symmetry: if $\alpha=a^{o}$ is any s-oriented corner on the real or imaginary line, and $\alpha^{\prime}=\left(a^{\prime}\right)^{o^{\prime}}$ is the rotated corner $a^{\prime}=e^{-\frac{\pi i}{2}} a \in \mathcal{V}_{\left[\mathbb{C}_{1}, 0\right]}^{c}$ oriented to $o^{\prime}=\left(e^{-\frac{\pi i}{4}} \sqrt{o}\right)^{2}$, and $z^{\prime}=e^{-\frac{\pi i}{2}} z$, we have,

$$
H_{\left[\mathbb{C}_{1}, 0\right]}^{\alpha}(z)=e^{-\frac{\pi i}{4}} H_{\left[\mathbb{C}_{1}, 0\right]}^{\alpha^{\prime}}\left(z^{\prime}\right) .
$$


Proof. Generalizing from the proof of Proposition 3.19, we construct a function on $\left[\mathbb{C}_{1}, 0\right]$ which decays at infinity and is s-holomorphic everywhere away from the specified singularity at $a$, and we argue that it is the unique function which can satisfy the properties specified in Theorem 3.15. For convenience, we will take source points $\alpha$ on the sheet $\mathbb{X}^{+}$unless otherwise specified; i.e. the positive real line approached from above on $\mathbb{Y}^{+}$.

Assume $a \in \mathcal{V}_{\left[\mathbb{C}_{1}, 0\right]}^{i} \cap \mathbb{R}_{>0}$ and consider the restriction $H^{\alpha}$ of $H_{\left[\mathbb{C}_{1}, 0\right]}^{\alpha}$ to the imaginary corners on the slit plane $\mathcal{V}_{\left[\mathbb{C}_{1}, 0\right]}^{i} \cap \mathbb{Y}^{+}$. The function $H^{\alpha}$ can be characterized as the unique discrete harmonic function on the slit plane $\mathcal{V}_{\left[\mathbb{C}_{1}, 0\right]}^{i} \cap \mathbb{Y}^{+}$which has the single-valued boundary data $H^{\alpha}(a):=-\frac{i}{2 \sqrt{2}} \sqrt{o}$ and zero elsewhere on the slit $\mathbb{R}_{>0}$, and decays at infinity. The harmonic function with these properties can be obtained by translating $H^{\alpha-2}$ for $\alpha-2:=(a-2)^{o}$ (which takes its only nonzero boundary value at $a-2$ ) to the right, and subtracting off a multiple of $H^{\alpha_{0}}$ in order to cancel the nonzero value at $a_{0}=\frac{1}{2}$. Specifically, letting

$$
\begin{aligned}
H^{\alpha}(z)= & H^{\alpha-2}(z-2)-H^{\alpha-2}\left(-\frac{3}{2}\right) \cdot \operatorname{hm}_{1 / 2}^{\mathbb{Y}_{1}^{i}}(z)=H^{\alpha-2}(z-2) \\
& -\operatorname{hm}_{a-2}^{\mathbb{Y}_{1}^{i}}\left(-\frac{3}{2}\right) \cdot H^{\alpha_{0}}(z),
\end{aligned}
$$

then discrete holomorphicity relations imply that the real part is determined up to a an additive constant; however since the real part must vanish on $\mathbb{R}_{<0}$, the real part is uniquely determined. In fact, it is easy to see that, in order to maintain discrete holomorphicity, the above recursive relation should also hold for the entire $H_{\left[\mathbb{C}_{1}, 0\right]}^{\alpha}$ as long as we are in the four real-translation invariant half-planes $\mathbb{X}^{ \pm} \cap \mathbb{Y}^{ \pm}$. For the explicit identification of the coefficients, we refer to Proposition A.2.

For $a \in \mathcal{V}_{\left[\mathbb{C}_{1}, 0\right]}^{1} \cap \mathbb{R}_{>0}$, we use a similar recursive process but now instead first construct the restriction $H^{\prime \alpha}$ of $H_{\left[\mathbb{C}_{1}, 0\right]}^{\alpha}$ to the real corners on $\mathbb{Y}^{+}$, starting from the case $a=\frac{3}{2}$. Unlike the imaginary case, we need to consider $-\frac{1}{2}$ as well as $\mathcal{V}_{\left[\mathbb{C}_{1}, 0\right]}^{1} \cap \mathbb{R}_{>0}$ as part of the slit boundary (where harmonicity fails): since $H_{\left[\mathbb{C}_{1}, 0\right]}^{\alpha}\left(\frac{1}{2}\right) \neq 0$ in general, we cannot assume that the real part is harmonic at $-\frac{1}{2}$ (see Remark 2.6).

In other words, $H^{\prime \alpha}$ is the function harmonic on the slit plane $\mathcal{V}_{\left[\mathbb{C}_{1}, 0\right]}^{1} \cap \mathbb{Y}^{+} \backslash\left\{-\frac{1}{2}\right\}$ which takes nonzero boundary values only at $a$ and $-\frac{1}{2}=-a_{0}$. As above $H^{\prime \alpha}(a)=-\frac{i}{2 \sqrt{2}} \sqrt{o}$.

For the value at $-a_{0}:=e^{\pi i} a_{0}$ with oriented version $-\alpha_{0}:=\left(-a_{0}\right)^{o^{\prime}}$, where $o^{\prime}=$ $\left(e^{\frac{\pi i}{2}}\right)^{2}$ we use antisymmetry in the two inputs to write $H_{\left[\mathbb{C}_{1}, 0\right]}^{\alpha}\left(-a_{0}\right)=-i \sqrt{o} H_{\left[\mathbb{C}_{1}, 0\right]}^{-\alpha_{0}}(a)$. Since by rotation $H_{\left[\mathbb{C}_{1}, 0\right]}^{-\alpha_{0}}(a)=-i H_{\left[\mathbb{C}_{1}, 0\right]}^{\alpha_{0}}\left(e^{-\pi i} a\right)$, we conclude $H_{\left[\mathbb{C}_{1}, 0\right]}^{\alpha}\left(-a_{0}\right)=$ $-\sqrt{o} H_{\left[\mathbb{C}_{1}, 0\right]}^{\alpha_{0}}\left(e^{-\pi i} a\right)=-\frac{i}{2 \sqrt{2}} \sqrt{o} \cdot \mathrm{hm}_{1 / 2}^{\mathbb{Y}_{1}^{i}}(-a)$. We can match these boundary values with recursion as above.

The general rotation identity can be verified independently with the same strategy, i.e., identifying the restriction of the left-hand side to a specific type of corner as the unique harmonic function with suitable boundary values, which the right-hand side solves.

Remark 3.23. The coefficients in Proposition 3.22 of various translated and rotated versions of $H_{\left[\mathbb{C}_{1}, 0\right]}^{\alpha_{0}}$ (with the same scaling limit) become important when identifying the scaling limit of the observables in Lemma 5.14. In particular, by Proposition A.2, the coefficients in the recursive expansion sum to zero in the case of $a \in \mathcal{V}_{\left[\mathbb{C}_{1}, 0\right]}^{1} \cap \mathbb{R}_{>0}$, which will yield that $\tilde{C}_{\alpha}=0=C_{\alpha}$ in Corollary 5.19. 


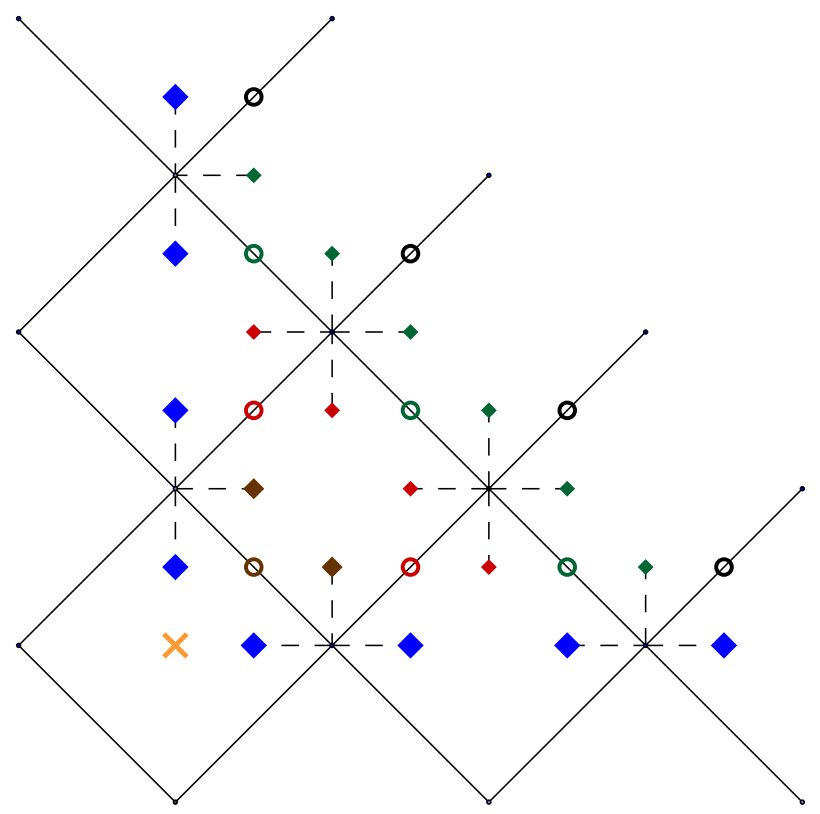

Fig. 5. The full plane spinor $H_{\left[\mathbb{C}_{1}, 0\right]}^{\alpha}(z)$ is first defined at all blue corners $\diamond$ by its antisymmetry relations and Proposition 3.22. By s-holomorphicity, we can then deduce its value at the brown medial vertex o, and then project from there onto the two brown corners $\downarrow$. We continue this process, next moving to the red $\circ$, then the red $\diamond$, and then green etc

Corollary 3.24. For any $a \in \mathcal{V}_{\left[\mathbb{C}_{1}, 0\right]}^{c m}$, we can recursively compute $H_{\left[\mathbb{C}_{1}, 0\right]}^{\alpha}$ as a finite linear combination of functions given by Theorem 3.21.

Proof. By Proposition 3.22, $H_{\left[\mathbb{C}_{1}, 0\right]}^{\alpha}(z)$ is given for all $z \in \mathcal{V}_{\left[\mathbb{C}_{1}, 0\right]}^{c m}$ whenever $\alpha \in \mathcal{V}_{\left[\mathbb{C}_{1}, 0\right]}^{1, i} \cap$ $\left(\mathbb{R}_{>0} \cup i \mathbb{R}_{>0}\right)$. The antisymmetry relations it satisfies thus give $H_{\left[\mathbb{C}_{1}, 0\right]}^{\alpha}(z)$ for every $\alpha$, whenever $z \in \mathbb{R}_{>0} \cup i \mathbb{R}_{>0}$ (as a finite linear combination of explicit harmonic measures).

Now observe that s-holomorphicity implies that the value, say, of $H_{\left[\mathbb{C}_{1}, 0\right]}^{\alpha}\left(\frac{1+i}{2}\right)$ can be recovered from its values at the $\frac{1}{2}, \frac{i}{2}$. From there, one can project the values of $H_{\left[\mathbb{C}_{1}, 0\right]}^{\alpha}(z)$ when $z=1+\frac{i}{2}$ and $\frac{1}{2}+i$ (see Fig. 5). Continuing this process allows for a recursive construction of $H_{\left[\mathbb{C}_{1}, 0\right]}^{\alpha}(z)$ for any $z \in \mathcal{V}_{\left[\mathbb{C}_{1}, 0\right]}^{c m}$ as a finite linear combination of the explicit functions of Theorem 3.21.

3.2.4. Auxiliary functions We introduce here full-plane auxiliary functions $G$ and $\tilde{G}^{ \pm}$, which are everywhere s-holomorphic functions on $\left[\mathbb{C}_{1}, 0\right]$ which do not decay at infinity. The real part of $G$ was defined in [CHI15] as a discrete version of the holomorphic function $\sqrt{z}$ on the double cover; we extend the result in order to give full s-holomorphic discrete representations of $\frac{1}{2 \sqrt{2}} \sqrt{z}$ and $\frac{i}{2 \sqrt{2}} \sqrt{z}$. Convergence results for these functions will be proved in Sect. 5.4.

As in previous subsections, we define the functions on the unit grid $\left[\mathbb{C}_{1}, 0\right]$, and then scale them by $G_{\left[\mathbb{C}_{\delta}, 0\right]}(z \delta):=\delta G_{\left[\mathbb{C}_{1}, 0\right]}(z)$ and $\tilde{G}_{\left[\mathbb{C}_{\delta}, 0\right]}^{ \pm}(z \delta):=\delta \tilde{G}_{\left[\mathbb{C}_{1}, 0\right]}^{ \pm}(z)$. We define them first on real and imaginary corners by "integrating" the harmonic measures, then extend to other points by s-holomorphicity. The fact that there are two discrete versions 
of $\frac{i}{2 \sqrt{2}} \sqrt{z}$ is a peculiarity that will be important in the proof of the main convergence result in Sect.5.4.2 (see also [Dub15]).

Definition 3.25. Define for $z \in \mathcal{V}_{\left[\mathbb{C}_{1}, 0\right]}^{1} \cup \mathcal{V}_{\left[\mathbb{C}_{1}, 0\right]}^{i}$, the auxiliary functions

$$
G_{\left[\mathbb{C}_{1}, 0\right]}(z):= \begin{cases}\sum_{n=0}^{\infty} \pm \frac{1}{2 \sqrt{2}} \mathrm{hm}_{3 / 2}^{\mathbb{X}_{1}^{1}(z-2 n)} & \text { if } z \in \mathcal{V}_{\left[\mathbb{C}_{1}, 0\right]}^{1} \cap \mathbb{X}^{ \pm} \\ \sum_{n=0}^{\infty} \pm \frac{i}{2 \sqrt{2}} \mathrm{hm}_{-3 / 2}^{\mathbb{Y}_{1}^{i}}(z+2 n) & \text { if } z \in \mathcal{V}_{\left[\mathbb{C}_{1}, 0\right]}^{i} \cap \mathbb{Y}^{ \pm}, \\ 0 & \text { otherwise }\end{cases}
$$

and

$$
\tilde{G}_{\left[\mathbb{C}_{1}, 0\right]}^{ \pm}(z):=i G_{\left[\mathbb{C}_{1}, 0\right]}(z \pm 1),
$$

where translation by 1 is well-defined at any point other than $\pm \frac{1}{2} ; G_{\left[\mathbb{C}_{1}, 0\right]}\left( \pm \frac{1}{2}\right)=0$ on both sheets so there is no ambiguity in defining $\tilde{G}_{\left[\mathbb{C}_{1}, 0\right]}^{ \pm}\left(\mp \frac{1}{2}\right)$.

Remark 3.26. In [CHI15, Lemma 2.17] well-definedness and harmonicity of the real part of $G_{\left[\mathbb{C}_{1}, 0\right]}$ were proven. From symmetry, we see the same holds for the imaginary part. Discrete holomorphicity of $G_{\left[\mathbb{C}_{1}, 0\right]}$, and thus of $\tilde{G}_{\left[\mathbb{C}_{1}, 0\right]}^{ \pm}$, is proved in Appendix A using the explicit formula of Theorem 3.21. Using that, we then extend these to s-holomorphic functions on the corners and medial vertices, again using the process of Remark 2.3.

Remark 3.27. Since $G_{\left[\mathbb{C}_{1}, 0\right]}$ (and thus $\tilde{G}_{\left[\mathbb{C}_{1}, 0\right]}^{ \pm}$) is defined using infinite sums, one cannot a priori calculate them exactly. However, once its s-holomorphicity is exhibited in Appendix A, we can use a propagation procedure similar to one shown in Fig. 5 and explained in Corollary 3.24 to recursively calculate its values from its values on the real and imaginary axes. Indeed, $G_{\left[\mathbb{C}_{1}, 0\right]}$ is explicitly computable on the real line since the summands eventually become zero; then we use the rotation identity $e^{\pi i / 4} G_{\left[\mathbb{C}_{1}, 0\right]}\left(e^{\pi i / 2} z\right)=\frac{1}{2}\left[\tilde{G}_{\left[\mathbb{C}_{1}, 0\right]}^{+}+\tilde{G}_{\left[\mathbb{C}_{1}, 0\right]}^{-}\right](z)$, proved in Proposition A.4, to find the values on the imaginary axis.

Using this procedure, we provide some explicit values of $G_{\left[\mathbb{C}_{1}, 0\right]}(z)$ near the origin in Fig. 6.

\section{Discrete Multipoint Observables}

In this section, we prove Pfaffian formulae expressing $n$-point energy correlations, with or without a spin weight, in terms of the real fermion and spin-fermion two-point functions.

For the $n$-point energy correlations, we formulate them in terms of s-holomorphic multipoint fermion observables introduced in [Hon10] and follow the strategy there to obtain their Pfaffian formulation with the two-point observables introduced in Sect. 3. In [Hon 10], the arguments of the multipoint observable were required to be distinct, nonadjacent medial vertices; we slightly but crucially generalize this to allow for adjacent medial vertices in Proposition 4.5 and Lemma 4.6, using a combinatorial correspondence between paths sourced at medial vertices and at corners.

When looking at $n$-point energy correlations weighted by a spin, the process is analogous, and we get fused multipoint spin-fermion observables, which then reduce to Pfaffians of the two-point spin-fermion observables of Sect.3.1. 


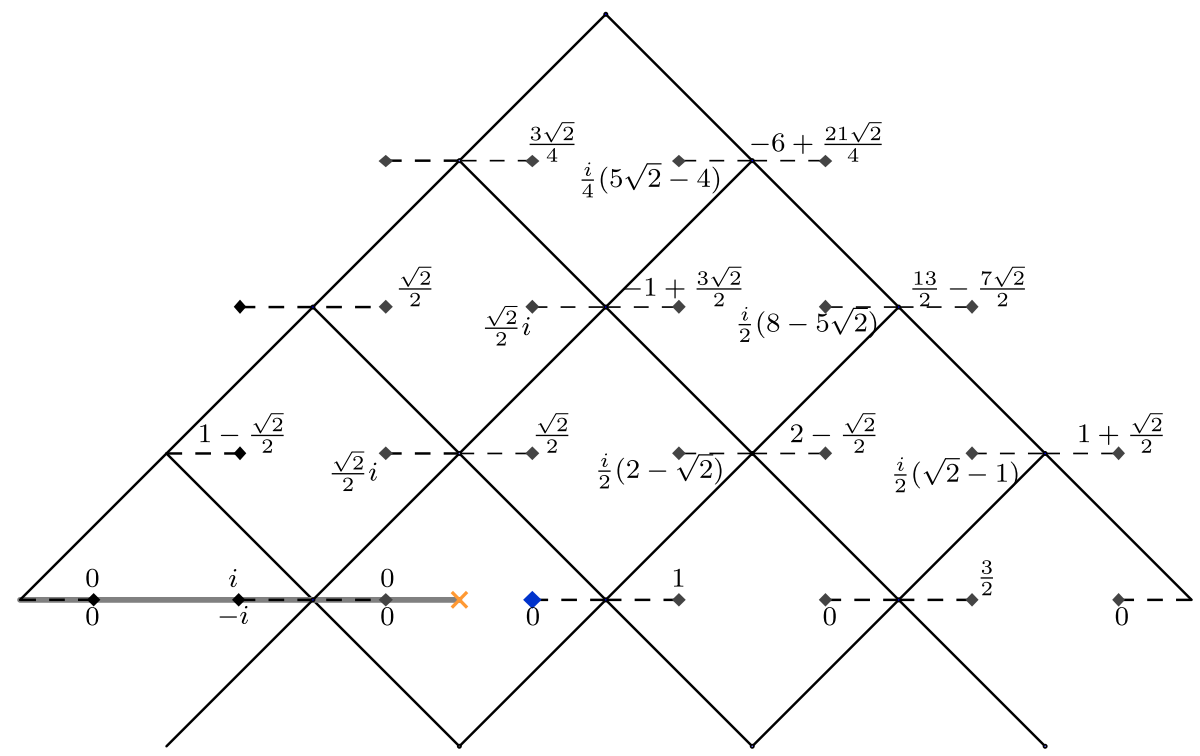

Fig. 6. Some explicit values for the auxiliary function $2 \sqrt{2} G_{\left[\mathbb{C}_{1}, 0\right]}(z)$ for $z \in \mathcal{V}_{\left[\mathbb{C}_{1}, 0\right]}^{1, i} \cap \mathbb{X}^{+}$, as defined in Definition 3.25. The function has monodromy about the origin, represented by the orange $x$

The proofs of these relations connecting the two-point fermion and spin-fermion observables to edge correlations (namely, Propositions 4.16-4.17) are quite notationally heavy, but all the extra notation is contained completely to this section. After generalizing the discrete complex analytic properties of the multipoint observables to adjacent medial vertices in Proposition 4.5, the rest of the proof is just a natural extension of the steps outlined in [Hon10] to prove the desired relations. For an alternative approach using mostly combinatorial arguments, see [CCK17].

4.1. Multipoint observables. Recall that in Sect. 3 we denoted by $\mathcal{Z}_{\Omega_{\delta}}$ the lowtemperature expansion of the partition function defined by summing $e^{-2 \beta|\omega|}$ over all $\omega \in \mathcal{C}_{\Omega_{\delta}}$, where $\mathcal{C}_{\Omega_{\delta}}$ is the set of closed loops in $\Omega_{\delta}$. Furthermore, for oriented corners or medial vertices $\alpha, \zeta$, we defined the contour sets $\mathcal{C}_{\Omega_{\delta}}^{\alpha, \zeta}$ as well as admissible walks. We also chose the convention that and at ambiguous vertices in the walk, we connect northeast to northwest edges and southeast and southwest edges (see Sect. 3.1).

We now generalize the two-point observables defined in Sect. 3 (refer also to Fig. 7). First, let us define the generalized contour set $\mathcal{C}_{\Omega_{\delta}}^{\alpha_{1}, \ldots, \alpha_{2 n}}$ for s-oriented corners or medial vertices $\alpha_{j}=a_{j}^{o_{j}}$ for $j=1,2, \ldots, 2 n$. For now we assume that the underlying points $a_{1}, \ldots, a_{2 n}$ are distinct (we will later generalize to the case where they can take the same value: see Remark 4.12). In [Hon10], these points were all medial vertices, and were required to be non-adjacent (the edges corresponding to them were not allowed to be adjacent). We do not impose this non-adjacency requirement, and this small extension is important to the proofs. As before, the half-edge of a corner is the line segment connecting it to its nearest vertex.

Each element $\gamma \in \mathcal{C}_{\Omega_{\delta}}^{\alpha_{1}, \ldots, \alpha_{2 n}}$ is a set containing 


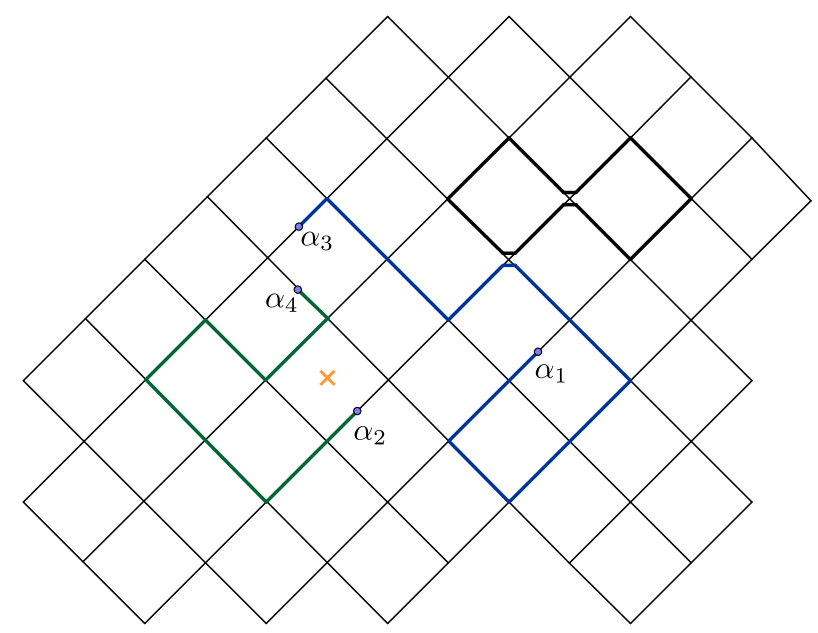

Fig. 7. An example of a choice of two admissible walks between $\alpha_{1}$ and $\alpha_{3}$ and $\alpha_{2}$ and $\alpha_{4}$ and a loop. The winding of $\gamma^{\alpha_{1}, \alpha_{3}}$ is $2 \pi$ while the winding of $\gamma^{\alpha_{2}, \alpha_{4}}$ is $-\frac{\pi}{2}$. The crossing parity has $\mathbf{c}(\Gamma(\gamma))=1$

- $2 n$ half-edges of $a_{1}, \ldots, a_{2 n}$ selected by their respective orientations $o_{1}, \ldots, o_{2 n}$, and

- a (possibly empty) collection of edges distinct from the above-mentioned half-edges of $\left\{a_{j}\right\}_{j=1}^{2 n}$,

such that any vertex of $\Omega_{\delta}$ is incident to an even number of the edges and half-edges. Such a $\gamma$ will be an edge-disjoint union of $n$ walks connecting $\alpha_{1}, \ldots \alpha_{2 n}$ pairwise as well as a (possibly empty) collection of edge-disjoint loops. In particular, the definition of the 2-point set $\mathcal{C}_{\Omega_{\delta}}^{\alpha, \zeta}$ coincides with the one given in Sect. 3 .

Generalizing from the 2-point case in Sect.3.0.2, given $\gamma$ we can pick $n$ admissible walks which connect $\left\{\alpha_{i}\right\}$ pairwise. We denote by $\Gamma(\gamma) \subset \gamma$, a set of those $n$ edgedisjoint admissible walks $\left\{\gamma^{\alpha_{j}, \alpha_{k}}\right\}$ chosen from the half-edges and edges constituting $\gamma$. We label them so that $j<k$ for each $\gamma^{\alpha_{j}, \alpha_{k}}$. Define the crossing parity $\mathbf{c}(\Gamma(\gamma))$ as the number of crossings, modulo 2 when linking $1, \ldots, 2 n \in \mathbb{R}$ pairwise with generic simple curves in the upper half plane (i.e. connect $j, k$ if there is a walk in $\Gamma(\gamma)$ connecting $\left.\alpha_{j}, \alpha_{k}\right)$. Moreover, recall the definitions of $\lambda_{\alpha_{i}, \alpha_{j}}$ and $\mathbf{W}(\gamma)$ from Definition 3.1.

Definition 4.1. For a collection of s-oriented corners or medial vertices $\left\{\alpha_{j}\right\}_{j=1}^{2 n}$ in $\Omega_{\delta}$, define the multipoint fermion observable as

$$
\begin{aligned}
F_{\Omega_{\delta}}^{\alpha_{1}, \ldots, \alpha_{2 n}} & :=\frac{1}{\mathcal{Z}_{\Omega_{\delta}}} \sum_{\gamma \in \mathcal{C}_{\Omega_{\delta}}^{\alpha_{1}, \ldots, \alpha_{2 n}}} \phi_{\left\{\alpha_{j}\right\}}(\gamma), \\
\phi_{\left\{\alpha_{j}\right\}}(\gamma) & :=e^{-2 \beta_{c}|\gamma|}(-1)^{\mathbf{c}(\Gamma(\gamma))} \prod_{\gamma^{\alpha_{j}, \alpha_{k}} \in \Gamma(\gamma)} i \lambda_{\alpha_{i}, \alpha_{j}} e^{-\frac{i}{2} \mathbf{W}\left(\gamma^{\alpha_{j}, \alpha_{k}}\right)} .
\end{aligned}
$$

In analogy with the two point case, if $\zeta^{1}=z^{p_{1}}$ and $\zeta^{2}=z^{p_{2}}$ are s-orientations of the two opposite orientations of $z:=a_{2 n}$, so that $p_{2}=e^{ \pm \pi i} p_{1}$, and $\kappa=c^{q}$ is an s-orientation of 
a corner $c$ in $\Omega_{\delta}$, we define the complexification of the multipoint fermion observable,

$$
\begin{aligned}
H_{\Omega_{\delta}}^{\alpha_{1}, \ldots, \alpha_{2 n-1}}(z) & :=\frac{1}{i \sqrt{p_{1}}} F_{\Omega_{\delta}}^{\alpha_{1}, \ldots, \alpha_{2 n-1}, \zeta^{1}}+\frac{1}{i \sqrt{p_{2}}} F_{\Omega_{\delta}}^{\alpha_{1}, \ldots, \alpha_{2 n-1}, \zeta^{2}}, \\
H_{\Omega_{\delta}}^{\alpha_{1}, \ldots, \alpha_{2 n-1}}(c) & :=\frac{1}{i \sqrt{q}} F_{\Omega_{\delta}}^{\alpha_{1}, \ldots, \alpha_{2 n-1}, \kappa} .
\end{aligned}
$$

Definition 4.2. For a collection of s-oriented corners or medial vertices $\left\{\alpha_{j}\right\}_{j=1}^{2 n}$ in $\left[\Omega_{\delta}, 0\right]$, define the multipoint spin-fermion observable,

$$
\begin{aligned}
& F_{\left[\Omega_{\delta}, 0\right]}^{\alpha_{1}, \ldots, \alpha_{2 n}}:=\frac{1}{\mathcal{Z}_{\Omega_{\delta}} \mathbb{E}_{\Omega_{\delta}}^{+}\left[\sigma_{0}\right]} \sum_{\gamma \in \mathcal{C}_{\Omega_{\delta}}^{\alpha_{1}, \ldots, \alpha_{2 n}}} \phi_{\left\{\alpha_{j}\right\}}^{\Sigma}(\gamma), \\
& \phi_{\left\{\alpha_{j}\right\}}^{\Sigma}(\gamma):=\phi_{\left\{\alpha_{j}\right\}}(\gamma)(-1)^{\ell(\gamma \backslash \cup \Gamma(\gamma))} \prod_{\gamma^{\alpha_{j}, \alpha_{k}} \in \Gamma(\gamma)} \mathrm{s}_{\alpha_{\mathrm{j}}, \alpha_{\mathrm{k}}}\left(\gamma^{\alpha_{j}, \alpha_{k}}\right),
\end{aligned}
$$

where $\ell$ and $\mathrm{s}_{\alpha_{\mathrm{j}}, \alpha_{\mathrm{k}}}$ are defined as in Definition 3.1. As in the two-point spin fermion, the contour collection $\mathcal{C}_{\Omega_{\delta}}^{\alpha_{1}, \ldots, \alpha_{2 n}}$ and the weight $\phi_{\left\{\alpha_{j}\right\}}$ are computed with respect to the projections of $\alpha_{j}$ onto $\Omega_{\delta}$, but the sheet choices come in the terms $\mathrm{s}_{\alpha, \zeta}$. Define the complexified spin-fermion $H_{\left[\Omega_{\delta}, 0\right]}^{\alpha_{1}, \ldots, \alpha_{2 n-1}}$ on medial vertices and corners of $\left[\Omega_{\delta}, 0\right]$ analogously to Eq. (4.1).

Remark 4.3 [Hon10, Propositions 67, 68] proves well-definedness of $\phi_{\left\{\alpha_{j}\right\}}(\gamma)$. In Proposition B.1 we prove the well-definedness of $(-1)^{\ell(\gamma \backslash \cup \Gamma(\gamma))} \prod \mathrm{s}_{\alpha_{\mathrm{j}}, \alpha_{\mathrm{k}}}\left(\gamma^{\alpha_{j}, \alpha_{k}}\right)$, and thus of $\phi_{\left\{\alpha_{j}\right\}}^{\Sigma}$, so that it is independent of our convention for admissible walks and the choice of pairings of source points; in particular, it is independent of our choice of admissible $\Gamma(\gamma)$.

In addition to increasing the number of inputs, we can also define observables summing over a subset $\mathcal{C}_{\Omega_{\delta}:\left\{e_{k}^{s_{k}}\right\}}^{\alpha_{1}, \ldots, \alpha_{2 n}} \subset \mathcal{C}_{\Omega_{\delta}}^{\alpha_{1}, \ldots, \alpha_{2 n}}$ formed by specifying the inclusion or exclusion of given edges. Given a collection of edges $\left\{e_{k}\right\}_{k=1}^{m}$ in $\Omega_{\delta}$ (disjoint to the half-edges given by $\left\{a_{j}\right\}_{i=1}^{2 n}$ ) and corresponding inclusion variables $s_{k} \in\{\bullet, \circ\}$, let

$$
\mathcal{C}_{\Omega_{\delta}:\left\{e_{1}, \ldots, e_{m}^{s_{m}}\right\}}^{\alpha_{1}, \ldots, \alpha_{2 n}}=\left\{\gamma \in \mathcal{C}_{\Omega_{\delta}}^{\alpha_{1}, \ldots, \alpha_{2 n}}: e_{k} \in \gamma \text { if } s_{k}=\bullet \text {, and } e_{k} \notin \gamma \text { if } s_{k}=0\right\} .
$$

Definition 4.4. We define the restricted fermion and spin-fermion observables, denoted $F_{\Omega_{\delta}:\left\{e_{1}^{s_{1}}, \ldots, e_{m}^{s_{m}}\right\}}^{\alpha_{1}, \ldots, \alpha_{2 n}}$ and $F_{\left[\Omega_{\delta}, 0\right]:\left\{e_{1}^{s_{1}}, \ldots, e_{m}^{s_{m}}\right\}}^{\alpha_{1}, \ldots, \alpha_{2 n}}$, and their complexifications as in Definitions 4.1 -4.2, replacing the contour set $\mathcal{C}_{\Omega_{\delta}}^{\alpha_{1}, \ldots, \alpha_{2 n}}$ by the restricted contour set $\mathcal{C}_{\Omega_{\delta}:\left\{e_{1}^{s_{1}}, \ldots, e_{m}^{s_{m}}\right\}}^{\alpha_{1}, \ldots, \alpha_{2 n}}$.

The following propositions will characterize the complexified fermion and spinfermion observables in terms of discrete complex analysis, proving the connection to the discrete Riemann boundary value problem defined in Sect. 2 for possibly adjacent medial vertices $a_{1}, \ldots, a_{2 n}$.

We first modify the real weight $\phi_{\left\{\alpha_{k}\right\}}$ defined on $\mathcal{C}_{\Omega_{\delta}}^{\alpha_{1}, \ldots, \alpha_{2 n}}$ for $2 n$ s-oriented vertices $\alpha_{1}, \ldots, \alpha_{2 n}$ into a complex weight $\chi$ defined on

$$
\mathcal{C}_{\Omega_{\delta}}^{\alpha_{1}, \ldots, \alpha_{2 n-1}, a_{2 n}}:=\mathcal{C}_{\Omega_{\delta}}^{\alpha_{1}, \ldots, \alpha_{2 n-1}, \alpha_{2 n}^{1}} \sqcup \mathcal{C}_{\Omega_{\delta}}^{\alpha_{1}, \ldots, \alpha_{2 n-1}, \alpha_{2 n}^{2}}
$$


for $2 n-1$ s-oriented medial vertices or corners $\alpha_{1}, \ldots, \alpha_{2 n-1}$ and another medial vertex $a_{2 n}$. Choose two s-orientations $\alpha_{2 n}^{1}=a_{2 n}^{p_{1}}$ and $\alpha_{2 n}^{2}=a_{2 n}^{p_{2}}$ of $a_{2 n}$ such that the orientations $p_{1}, p_{2}$ are the two opposite permissible orientations on $a_{2 n}$. Define for $\gamma \in \mathcal{C}_{\Omega_{\delta}}^{\alpha_{1}, \ldots, \alpha_{2 n}^{j}} \subset \mathcal{C}_{\Omega_{\delta}}^{\alpha_{1}, \ldots, \alpha_{2 n-1}, a_{2 n}}$ the complex weight (where dependence on the choice of $\sqrt{p_{j}}$ eventually cancels out),

$$
\chi(\gamma):=\frac{1}{i \sqrt{p_{j}}} \phi_{\left\{a_{1}, \ldots, \alpha_{2 n-1}, a_{2 n}^{j}\right\}}(\gamma),
$$

noting that the complexified observables can be defined in terms of sums of this weight. If $\alpha_{2 n}=a_{2 n}^{o}$ is an s-oriented corner, there is only one corresponding orientation and $\chi(\gamma):=\frac{1}{i \sqrt{o}} \phi_{\left\{\alpha_{k}\right\}}(\gamma)$.

Proposition 4.5. $H_{\Omega_{\delta}:\left\{e_{1}^{\left.s_{1}, \ldots, e_{m}\right\}}\right.}^{\alpha_{1}, \ldots, \alpha_{2 n-1}}$ and $H_{\left[\Omega_{\delta}, 0\right]:\left\{e_{1}^{s_{1}}, \ldots, e_{m}^{s_{m}}\right\}}^{\alpha_{1}, \ldots, \alpha_{2 n-1}}$ are s-holomorphic wherever defined.

Proof. This was proven for the complexified fermion in [Hon10, Lemma 74] in the setting where the $\alpha_{i}$ are non-adjacent medial vertices. We extend this to all possible $\alpha_{i}$ via the extension of the complexified fermion to corners in addition to medial vertices. The idea is that there is a natural bijection between the set of paths to a medial vertex $e$ and those to an adjacent corner $c$. Namely, if $e(c)$ is the shortest walk from $a_{2 n}$ to $c$ consisting of two half-edges both incident to the common vertex $v$, the map $\gamma \mapsto \gamma \oplus e(c)$ is a bijection. One needs to show that the projection in s-holomorphicity relations (2.1) sends the winding weight $e^{-\frac{i}{2} \mathbf{W}(\gamma)}$ to the winding weight of the image, times a factor of $\cos (\pi / 8)$.

Following this, the next lemma therefore proves s-holomorphicity in the case of $H_{\Omega_{\delta}:\left\{e_{1}^{s_{1}}, \ldots, e_{m}^{s_{m}}\right\}}^{\alpha_{1}, \ldots, \alpha_{2 n-1}}$. The summands in the definition of $H_{\left[\Omega_{\delta}, 0\right]:\left\{e_{1}^{s_{1}}, \ldots, e_{m}^{s_{m}}\right\}}^{\alpha_{1}, \ldots, \alpha_{2 n-1}}$ only have additional real factors invariant under the $\oplus e(c)$ bijection, so the lemma implies the sholomorphicity for $H_{\left[\Omega_{\delta}, 0\right]:\left\{e_{1}^{\left.s_{1}, \ldots, e_{m}^{s_{m}}\right\}}\right.}^{\alpha_{1}, \ldots, \alpha_{2 n-1}}$ as well.

Lemma 4.6. Let $\left\{a_{j}\right\}_{j=1}^{2 n-1}$, $\left\{e_{k}\right\}_{k=1}^{m}$ be distinct medial vertices of $\Omega_{\delta}$ and denote the $s$ oriented versions of $\left\{a_{j}\right\}_{j=1}^{2 n-1}$ by $\alpha_{j}=a_{j}^{o_{j}}$, and inclusion variables $s_{1}, \ldots, s_{m} \in\{\bullet, \circ\}$. Suppose $c$ is an interior corner and $a_{2 n}$ is an adjacent interior medial vertex distinct from $\left\{a_{j}\right\}_{j=1}^{2 n-1}$. Let $e(c)$ be the shortest walk from $a_{2 n}$ to c consisting of two half-edges both incident to a common vertex $v$. Then the bijection,

$$
\gamma \mapsto \gamma \oplus e(c)
$$

from $\mathcal{C}_{\Omega_{\delta}:\left\{e_{k}^{s_{k}}\right\}}^{\alpha_{1}, \ldots, \alpha_{2 n-1}, a_{2 n}}$ to $\mathcal{C}_{\Omega_{\delta}:\left\{e_{k}^{s_{k}}\right\}}^{\alpha_{1}, \ldots, \alpha_{2 n-1}, c}$ satisfies the projection relation,

$$
\mathrm{P}_{l(c)} \chi(\gamma)=\chi(\gamma \oplus e(c))
$$

Proof. Suppose $\gamma_{f} \in \Gamma(\gamma)$ is the walk ending at $a_{2 n}$, starting at some $\alpha_{s}$. Suppose $c$ is a corner of type $\tau$ adjacent to $a_{2 n}$. Then $\Gamma(\gamma \oplus e(c))$, chosen using paths in $\Gamma(\gamma)$ with $\gamma_{f}$ replaced by $\gamma_{f} \oplus e(c)$, is clearly an admissible choice of walks in $\gamma \oplus e(c)$. Note that 
if $o_{2 n}$ is any s-orientation of $a_{2 n}$ compatible with $\gamma_{f}, i \frac{\sqrt{o_{2 n}}}{\sqrt{o_{s}}} e^{-\frac{i}{2} \mathbf{W}\left(\gamma_{f}\right)}$ is a real quantity. Thus it suffices to show that

$$
\mathrm{P}_{l(c)} \frac{e^{-2 \beta_{c}|\gamma|}}{\sqrt{o_{s}}} e^{-\frac{i}{2} \mathbf{W}\left(\gamma_{f}\right)}=\cos \frac{\pi}{8} \cdot \frac{e^{-2 \beta_{c}|\gamma \oplus e(c)|}}{\sqrt{o_{s}}} e^{-\frac{i}{2} \mathbf{W}\left(\gamma_{f} \oplus e(c)\right)} .
$$

Commuting real quantities with projections, we may rewrite the left hand side as

$$
i \frac{\sqrt{o_{2 n}}}{\sqrt{o_{S}}} e^{-\frac{i}{2} \mathbf{W}\left(\gamma_{f}\right)} e^{-2 \beta_{c}|\gamma|} \mathrm{P}_{l(c)} \frac{1}{i \sqrt{o_{2 n}}} .
$$

There are two cases to consider: the cases when the half-edge $\left\langle a_{2 n}, v\right\rangle \in \gamma_{f}$ and when $\left\langle a_{2 n}, v\right\rangle \notin \gamma_{f}$.

- In the first case, $|\gamma \oplus e(c)|=|\gamma|$. Subsequently $\tau^{2} o_{2 n}=-e^{ \pm \frac{\pi}{4} i}$, where the sign depends on the winding change $\mathbf{W}(\gamma \oplus e(c))=\mathbf{W}(\gamma) \mp \frac{\pi}{4}$. Now we have

$$
\mathrm{P}_{l(c)} \frac{1}{i \sqrt{o_{2 n}}}=\frac{1-\tau^{2} o_{2 n}}{2 i \sqrt{o_{2 n}}}=\frac{e^{ \pm \frac{\pi}{8} i}}{i \sqrt{o_{2 n}}} \cos \frac{\pi}{8},
$$

and the result follows.

- In the second case, $|\gamma \oplus e(c)|=|\gamma|+1$. Then $\tau^{2} o_{2 n}=e^{ \pm \frac{\pi}{4} i}$, where the sign depends on the winding change $\mathbf{W}(\gamma \oplus e(c))=\mathbf{W}(\gamma) \pm \frac{3 \pi}{4}$. Then

$$
\mathrm{P}_{l(c)} \frac{1}{i \sqrt{o_{2 n}}}=\frac{1-\tau^{-2} o_{2 n}}{2 i \sqrt{o_{2 n}}}=\frac{e^{\mp \frac{3 \pi}{8} i}}{i \sqrt{o_{2 n}}} \sin \frac{\pi}{8} .
$$

Upon noting that $\tan \frac{\pi}{8}=e^{-2 \beta_{c}}$, the result follows.

Lemma 4.7. The discrete residues at an s-oriented interior oriented medial vertex $\alpha_{j}$ are

$$
\begin{aligned}
& H_{\Omega_{\delta}:\left\{e_{1}^{\left.s_{1}, \ldots, e_{m}^{s_{m}}\right\}}\right.}^{\alpha_{1}, \ldots, \alpha_{2 n-1}}\left(\alpha_{j+}\right)-H_{\Omega_{\delta}:\left\{e_{1}^{\left.s_{1}, \ldots, e_{m}^{s_{m}}\right\}}\right.}^{\alpha_{1}, \ldots, \alpha_{2 n-1}}\left(\alpha_{j-}\right)=\frac{(-1)^{j+1}}{\sqrt{o_{j}}} F_{\Omega_{\delta}:\left\{e_{1}^{s_{1}}, \ldots, e_{m}^{s_{m}}\right\}}^{\alpha_{1}, \ldots, \alpha_{j-1}, \alpha_{j+1}, \ldots, \alpha_{2 n-1}}, \\
& H_{\left[\Omega_{\delta}, 0\right]:\left\{e_{1}^{\left.s_{1}, \ldots, e_{m}^{s_{m}}\right\}}\right.}^{\alpha_{1}, \ldots, \alpha_{2 n-1}}\left(\alpha_{j+}\right)-H_{\left[\Omega_{\delta}, 0\right]:\left\{e_{1}^{s_{1}}, \ldots, e_{m}^{s_{m}}\right\}}^{\alpha_{1}, \ldots, \alpha_{2 n-1}}\left(\alpha_{j-}\right)=\frac{(-1)^{j+1}}{\sqrt{o_{j}}} F_{\left[\Omega_{\delta}, 0\right]:\left\{e_{1}^{s_{1}}, \ldots, e_{m}^{s_{m}}\right\}}^{\alpha_{1}, \ldots, \alpha_{2 n-1}} .
\end{aligned}
$$

where in the two cases $\alpha_{1}, \ldots, \alpha_{2 n-1}$ are all taken respectively on $\Omega_{\delta}$ and $\left[\Omega_{\delta}, 0\right]$.

Proof. We prove this for the fermion and spin-fermion simultaneously, letting $D_{\delta}$ represent $\Omega_{\delta}$ or $\left[\Omega_{\delta}, 0\right]$. It suffices to show that the front and back values of $H_{D_{\delta}:\left\{e_{1}^{s_{1}}, \ldots, e_{m}^{\alpha_{m}}\right\}}^{\alpha_{1}, \ldots, \alpha_{2 n-1}}$ at $\alpha_{j}$ are given by

$$
\begin{aligned}
& H_{D_{\delta}:\left\{e_{1}^{s_{1}}, \ldots, e_{m}^{s_{m}}\right\}}^{\alpha_{1}, \ldots, \alpha_{2 n-1}}\left(\alpha_{j+}\right)=\frac{(-1)^{j+1}}{\sqrt{o_{j}}} F_{D_{\delta}:\left\{e_{1}^{s_{1}}, \ldots, e_{m}^{s_{m}}, a_{j}^{\circ}\right\}}^{\alpha_{1}, \ldots, \alpha_{j-1}, \alpha_{j+1}, \ldots \alpha_{2 n-1}}, \\
& H_{D_{\delta}:\left\{e_{1}^{s_{1}}, \ldots, e_{m}^{\left.s_{m}\right\}}\right.}^{\alpha_{1}, \ldots, \alpha_{2 n-1}}\left(\alpha_{j-}\right)=\frac{(-1)^{j}}{\sqrt{\sigma_{j}}} F_{D_{\delta}:\left\{e_{1}^{s_{1}}, \ldots, e_{m}^{s_{m}}, a_{j}^{\bullet}\right\}}^{\alpha_{1}, \ldots, \alpha_{j-1}, \alpha_{j+1}, \ldots \alpha_{2 n-1}},
\end{aligned}
$$


where in the case $D_{\delta}=\left[\Omega_{\delta}, 0\right]$ the projection of $a_{j}$ onto $\Omega_{\delta}$ is considered in $a_{j}^{\mathrm{o}, \bullet}$.

Let $c_{1}$ denote one of the two corners adjacent to the end vertex of $a_{j}$ in the direction

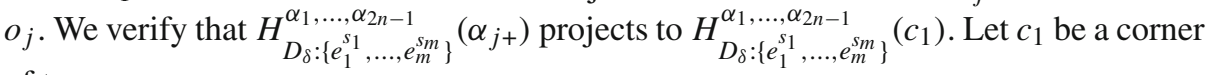
of type $\tau$.

We note that any $\left.\gamma \in \mathcal{C}_{D_{\delta}:\left\{e_{1}^{\left.s_{1}, \ldots, e_{m}^{s_{m}}\right\}}\right.}^{\alpha_{1}, \ldots, \alpha_{2 n-1}, c_{1}}\right\}$ contains the walk $e\left(c_{1}\right):=\left\{\left\langle a_{j}, a_{j}+o_{j} \frac{\delta}{\sqrt{2}}\right\rangle\right.$, $\left.\left\langle a_{j}+o_{j} \frac{\delta}{\sqrt{2}}, c_{1}\right)\right\}$, so we can start from $\gamma_{f}$ and complete $\Gamma(\gamma)$. In addition, $\gamma \oplus e\left(c_{1}\right) \in$ $\mathcal{C}_{D_{\delta}:\left\{e_{1}^{s_{1}, \ldots, s_{m}, a_{j}^{\circ}}\right.}^{\alpha_{1}, \ldots, \alpha_{j-1}, \alpha_{j+1}, \ldots, \alpha_{2 n-1}}$ and $\Gamma(\gamma) \backslash\left\{e\left(c_{1}\right)\right\}$ is naturally an admissible choice of walk for $2 n-2$ points. Since the additional real factor in the case of $D_{\delta}=\left[\Omega_{\delta}, 0\right]$ is easily seen to be invariant under the bijection, it now suffices to show the projection relation

$$
\mathrm{P}_{l\left(c_{1}\right)} \frac{(-1)^{j+1}}{\sqrt{o_{j}}} \phi_{\left\{\alpha_{1}, \ldots, \alpha_{j-1}, \alpha_{j+1}, \ldots, \alpha_{2 n-1}\right\}}\left(\gamma \oplus e\left(c_{1}\right)\right)=\chi(\gamma) .
$$

This relation can be rewritten as $\mathrm{P}_{l\left(c_{1}\right)} \frac{1}{\sqrt{o_{j}}}=e^{-2 \beta_{c}} \cos \frac{\pi}{8} \cdot \frac{e^{-\frac{i}{2} \mathrm{~W}\left(e\left(c_{1}\right)\right)}}{\sqrt{\sigma_{j}}}=\cos \frac{3 \pi}{8}$. $\frac{e^{-\frac{i}{2} \mathbf{W}\left(e\left(c_{1}\right)\right)}}{\sqrt{o_{j}}}$ using explicit formulae and admissible choices $\Gamma(\gamma)$ and $\Gamma\left(\gamma \oplus e\left(c_{1}\right)\right)$ (where $(-1)^{j+1}$ is precisely the ratio between the crossing parity factor of $\Gamma\left(\gamma \oplus e\left(c_{1}\right)\right)$ and $\Gamma(\gamma)$; one sees this by drawing the pairing between $j$ and $2 n$ very close to $[j, 2 n] \subset \mathbb{R}$, so that it crosses exactly $2 n-j-1$ other lines).

Note that $o_{j} e^{i \mathbf{W}\left(e\left(c_{1}\right)\right)}=\tau^{2}$ and $\mathbf{W}\left(e\left(c_{1}\right)\right)= \pm \frac{3 \pi}{4}$. Commuting real values with projection, we have,

$$
\mathrm{P}_{l\left(c_{1}\right)}\left[\frac{1}{\sqrt{o_{j}}}\right]=\frac{1}{2}\left[\frac{1}{\sqrt{o_{j}}}+\frac{\sqrt{o_{j}}}{\tau^{2}}\right]=\frac{1}{\sqrt{o_{j}}} \frac{1+e^{-i \mathbf{W}\left(e\left(c_{1}\right)\right)}}{2}=\cos \frac{3 \pi}{8} \cdot \frac{e^{-\frac{i}{2} \mathbf{W}\left(e\left(c_{1}\right)\right)}}{\sqrt{o_{j}}} .
$$

Remark 4.8. The explicit front and back values (4.2) shown in the proof above give us a simple correspondence between the full-plane observables and the full-plane (normal and spin-weighted) nearest-pair correlations $\mu, \mu_{a^{\prime}}$ for edges $a=\delta a^{\prime} \in \mathcal{E}_{\Omega_{\delta}}$ defined in (1.1).

Notice that, by low-temperature expansion, the correlation of the nearest spin pair separated by an edge $a$ can be written $\frac{1}{\mathcal{Z}_{\Omega_{\delta}}}\left[\sum_{\omega \in \mathcal{C}_{\Omega_{\delta}:\left\{a^{\circ}\right\}}} e^{-2|\omega|}-\sum_{\omega \in \mathcal{C}_{\Omega_{\delta}:\left\{a^{\bullet}\right\}}} e^{-2|\omega|}\right]=$ $F_{\Omega_{\delta}:\left\{a^{\circ}\right\}}-F_{\Omega_{\delta}:\left\{a^{\bullet}\right\}}$. By the values given in (4.2), this is precisely $\sqrt{o}\left[H_{\Omega_{\delta}}^{\alpha}\left(\alpha_{+}\right)+\right.$ $\left.H_{\Omega_{\delta}}^{\alpha}\left(\alpha_{-}\right)\right]$, for any s-oriented version $\alpha$ of $a$. Now taking the infinite-volume limit by sending $\Omega \uparrow \mathbb{C}$ (whose existence is known by Theorem 3.13), we have

$$
\mu=\sqrt{o}\left[H_{\mathbb{C}_{\delta}}^{\alpha}\left(\alpha_{+}\right)+H_{\mathbb{C}_{\delta}}^{\alpha}\left(\alpha_{-}\right)\right],
$$

matching the values $H_{\mathbb{C}_{\delta}}^{\alpha}\left(\alpha_{ \pm}\right)=\frac{\mu \pm 1}{2 \sqrt{o}}$ given by Proposition 3.14.

By analogous reasoning, for every $a=\delta a^{\prime} \in \mathcal{E}_{\Omega_{\delta}}$ we can calculate $\mu_{a^{\prime}}$ as defined in (1.1) by

$$
\mu_{a^{\prime}}=\sqrt{o}\left[H_{\left[\mathbb{C}_{\delta}, 0\right]}^{\alpha}\left(\alpha_{+}\right)+H_{\left[\mathbb{C}_{\delta}, 0\right]}^{\alpha}\left(\alpha_{-}\right)\right]
$$




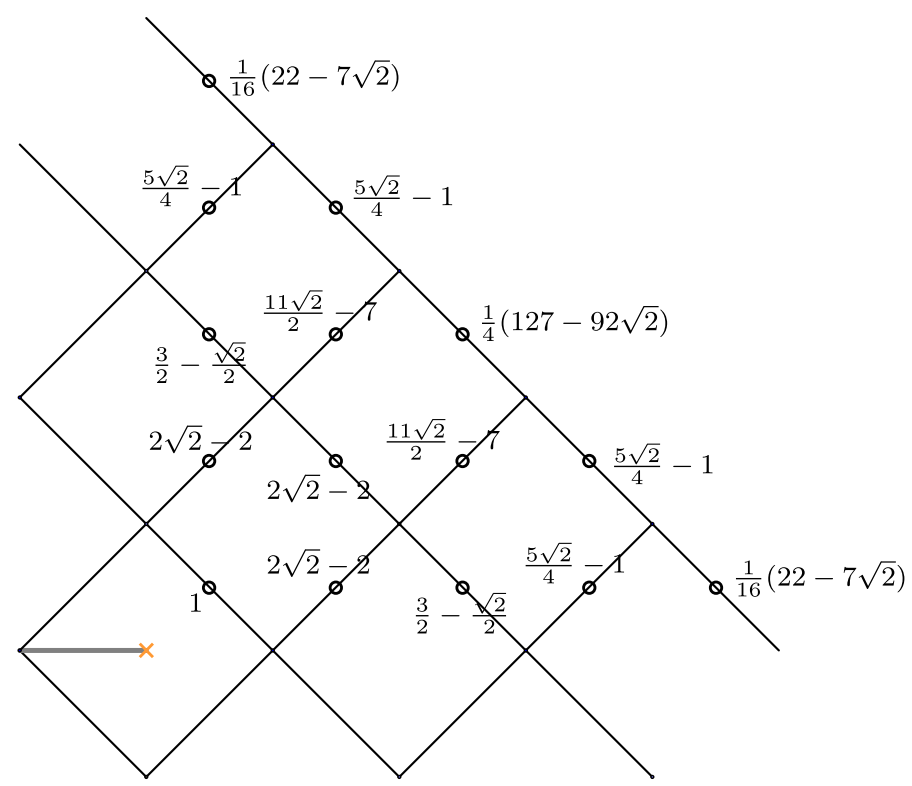

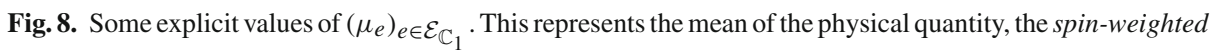
energy field. The orange $\times$ marks the identified origin face, whose spin $\mu_{e}$ is weighted by; as $|e| \rightarrow \infty$, we have $\mu_{e} \rightarrow \mu$

or equivalently for every $a=\delta a^{\prime} \in \mathcal{E}_{\Omega_{\delta}}$ we have $H_{\left[\mathbb{C}_{\delta}, 0\right]}^{\alpha}\left(\alpha_{ \pm}\right)=\frac{\mu_{a^{\prime}} \pm 1}{2 \sqrt{o}}$, where we know the infinite-volume limit $\Omega \uparrow \mathbb{C}$ of $\sqrt{o}\left[H_{\left[\Omega_{\delta}, 0\right]}^{\alpha}\left(\alpha_{+}\right)+H_{\left[\Omega_{\delta}, 0\right]}^{\alpha}\left(\alpha_{-}\right)\right]$exists by Theorem 3.15 (see Fig. 8 for some explicit values).

Recall the definition of $v_{z}$ as the outer normal at a boundary medial vertex $z$.

Lemma 4.9. If $\left\{\alpha_{i}\right\}_{i=1}^{2 n-1}$ are interior s-oriented medial vertices, $\left\{e_{1}^{s_{1}}, \ldots e_{m}^{s_{m}}\right\}$ are edges with corresponding inclusion variables, and $z=a_{2 n}$ is a boundary medial vertex, we have that

$$
\begin{gathered}
H_{\Omega_{\delta}:\left\{e_{1}^{s_{1}, \ldots, e_{m}}\right\}}^{\left.\alpha_{1}, \ldots, \alpha_{m}\right\}}(z) \sqrt{\nu_{z}} \in \mathbb{R} ; \\
H_{\left[\Omega_{\delta}, 0\right]:\left\{e_{1}^{s_{1}, \ldots, e_{m}}\right\}}^{\alpha_{1}, \ldots, \alpha_{2 n-1}}(z) \sqrt{\nu_{z}} \in \mathbb{R} .
\end{gathered}
$$

Proof. [Hon10, Proposition 79] proves the lemma for $H_{\Omega_{\delta}:\left\{e_{1}^{s_{1}}, \ldots, e_{m}^{s_{m}}\right\}}^{\alpha_{1}, \ldots, \alpha_{2 n-1}}$ with non-adjacent $\alpha_{i}$. The idea is that if $z$ is on the boundary, a path can only reach $z$ via the half-edge in the inner direction, which fixes the complex phase in the weight; this goes through unchanged for possibly adjacent $\alpha_{i}$. For the spin-fermion observable, since the additional factors in $H_{\left[\Omega_{\delta}, 0\right]:\left\{e_{1}^{s_{1}}, \ldots, e_{m}^{s_{m}}\right\}}^{\alpha_{1}, \ldots, \alpha_{2 n-1}}$ are real, the result holds.

4.2. Fused observables and Ising correlations. In this subsection, we formulate the Ising correlations in a bounded domain in terms of fusions of the observables introduced above. We again write $\alpha_{1}, \ldots, \alpha_{2 n}$ for distinct s-oriented medial vertices in $\Omega_{\delta}$ or $\left[\Omega_{\delta}, 0\right]$ and $e_{1}, \ldots, e_{m}$ distinct edges with inclusion variables $s_{1}, \ldots, s_{m} \in\{\bullet, \circ\}$. 
Definition 4.10. Suppose $b_{1}, \ldots, b_{N}$ are distinct medial vertices in $\Omega_{\delta}$ with $b_{N}=\delta b_{N}^{\prime}$. Define the fused fermion and fused spin-fermion observables and their complexifications inductively by

$$
\begin{aligned}
& F_{\Omega_{\delta}:\left\{e_{1}^{s_{1}}, \ldots, e_{m}^{s_{m}}\right\}}^{\alpha_{1}, \ldots, \alpha_{2 n}:\left[b_{1}, \ldots, b_{N}\right]}:=F_{\Omega_{\delta}:\left\{e_{1}^{s_{1}}, \ldots, e_{m}^{s_{m}}, b_{N}^{\circ}\right\}}^{\alpha_{1}, \ldots, \alpha_{2 n}:\left[b_{1}, \ldots, b_{N-1}\right]}-\frac{\mu+1}{2} F_{\Omega_{\delta}:\left\{e_{1}^{s_{1}, \ldots,}, e_{m}^{s_{m}}\right\}}^{\alpha_{1}, \ldots, \alpha_{2 n}:\left[b_{1}, \ldots, b_{N-1}\right]}, \\
& F_{\left[\Omega_{\delta}, 0\right]:\left\{e_{1}^{s_{1}}, \ldots, e_{m}^{s_{m}}\right\}}^{\alpha_{1}, \ldots, \alpha_{2 n}:\left[b_{1}, \ldots, b_{N}\right]}:=F_{\left[\Omega_{\delta}, 0\right]:\left\{e_{1}^{s_{1}}, \ldots, e_{m}^{s_{m}}, b_{N}^{\circ}\right\}}^{\alpha_{1}, \ldots, \alpha_{2 n}:\left[b_{1}, \ldots, b_{N-1}\right]}-\frac{\mu_{b_{N}^{\prime}+1}+1}{2} F_{\left[\Omega_{\delta}, 0\right]:\left\{e_{1}^{s_{1}}, \ldots, e_{m}^{s_{m}}\right\}}^{\alpha_{1}, \ldots, \alpha_{2 n}:\left[b_{1}, \ldots, b_{N-1}\right]}
\end{aligned}
$$

and the usual complexification scheme on $\alpha_{2 n}$ (see e.g. (4.1)).

These fusions arise naturally from the process of removing singularities. Suppose $\alpha_{j}$ is an s-oriented interior medial vertex. Note that, by Lemma 4.7 and Theorems 3.13-3.15, the functions

$$
\begin{aligned}
& H_{\Omega_{\delta}:\left\{e_{1}^{s_{1}, \ldots, e_{m}}\right.}^{\left.\alpha_{1}, \ldots, \alpha_{2 n-1}\right\}}+(-1)^{j} F_{\Omega_{\delta}:\left\{e_{1}^{s_{1}, \ldots, e_{m}} \alpha_{1}, \ldots, \alpha_{j-1}, \alpha_{j+1}, \ldots, \alpha_{2 n-1}\right.}^{\alpha_{1}} H_{\mathbb{C}_{\delta}}^{\alpha_{j}}, \\
& H_{\left[\Omega_{\delta}, 0\right]:\left\{e_{1}^{s_{1}}, \ldots, e_{m}^{s_{m}}\right\}}^{\alpha_{1}, \ldots, \alpha_{2 n-1}}+(-1)^{j} F_{\left[\Omega_{\delta}, 0\right]:\left\{e_{1}^{\left.s_{1}, \ldots, e_{m}^{s_{m}}\right\}}\right.}^{\alpha_{1}, \ldots, \alpha_{j-1}, \alpha_{j+1}, \ldots, \alpha_{2 n-1}} H_{\left[\mathbb{C}_{\delta}, 0\right]}^{\alpha_{j}},
\end{aligned}
$$

have discrete residue 0 at $\alpha_{j}$; thus they extend to $a_{j}$ s-holomorphically. In fact, we have the following extension result for them, which also applies to the corresponding fused observables.

Lemma 4.11. The following fused versions of the functions defined in (4.4)-(4.5) have s-holomorphic extensions to $a_{j}$ given by the values

$$
\begin{aligned}
& {\left[H_{\Omega_{\delta}}^{\alpha_{1}, \ldots, \alpha_{2 n-1}:\left[e_{1}, \ldots, e_{m}\right]}+(-1)^{j} F_{\Omega_{\delta}}^{\alpha_{1}, \ldots, \alpha_{j-1}, \alpha_{j+1}, \ldots, \alpha_{2 n-1}:\left[e_{1}, \ldots, e_{m}\right]} H_{\mathbb{C}_{\delta}}^{\alpha_{j}}\right]\left(a_{j}\right)} \\
& :=\frac{(-1)^{j+1}}{\sqrt{o_{j}}} F_{\Omega_{\delta}}^{\alpha_{1}, \ldots, \alpha_{j-1}, \alpha_{j+1}, \ldots \alpha_{2 n-1}:\left[e_{1}, \ldots, e_{m}, a_{j}\right]}, \\
& {\left[H_{\left[\Omega_{\delta}, 0\right]}^{\alpha_{1}, \ldots, \alpha_{2 n-1}:\left[e_{1}, \ldots, e_{m}\right]}+(-1)^{j} F_{\left[\Omega_{\delta}, 0\right]}^{\alpha_{1}, \ldots, \alpha_{j-1}, \alpha_{j+1}, \ldots, \alpha_{2 n-1}:\left[e_{1}, \ldots, e_{m}\right]} H_{\left[\mathbb{C}_{\delta}, 0\right]}^{\alpha_{j}}\right]\left(a_{j}\right)} \\
& :=\frac{(-1)^{j+1}}{\sqrt{o_{j}}} F_{\left[\Omega_{\delta}, 0\right]}^{\alpha_{1}, \ldots, \alpha_{j-1}, \alpha_{j+1}, \ldots \alpha_{2 n-1}:\left[e_{1}, \ldots, e_{m}, a_{j}\right]},
\end{aligned}
$$

where in the second case, $a_{j}$ in $\left[\Omega_{\delta}, 0\right]$ is identified with its projection on $\Omega_{\delta}$ in the expression $\left[e_{1}, \ldots, e_{m}, a_{j}\right]$.

Proof. By $\mathbb{R}$-linearity of s-holomorphicity and the definition of fused observables, it suffices to show that the unfused observables given by (4.4)-(4.5) extend s-holomorphically to $a_{j}$ with above right hand side values (without $e_{1}, \ldots, e_{m}$ ).

Since the function (4.4) has discrete residue 0 at $a_{j}$, it has an s-holomorphic extension to $a_{j}$ given by

$$
\begin{aligned}
& {\left[H_{\Omega_{\delta}:\left\{e_{1}^{s_{1}, \ldots, e_{m}} \alpha_{m}\right\}}^{\alpha_{1}, \ldots, \alpha_{2 n-1}}+(-1)^{j} F_{\Omega_{\delta}:\left\{e_{1}^{s_{1}}, \ldots, e_{m}^{s_{m}}\right\}}^{\alpha_{1}, \ldots, \alpha_{j-1}, \alpha_{j+1}, \ldots, \alpha_{2 n-1}} H_{\mathbb{C}_{\delta}}^{\alpha_{j}}\right]\left(a_{j}\right)} \\
& =H_{\Omega_{\delta}:\left\{e_{1}^{\left.s_{1}, \ldots, e_{m}^{s_{m}}\right\}}\right.}^{\alpha_{1}, \ldots, \alpha_{2 n-1}}\left(\alpha_{j+}\right)+(-1)^{j} F_{\Omega_{\delta}:\left\{e_{1}^{\left.s_{1}, \ldots, e_{m}^{s_{m}}\right\}}\right.}^{\alpha_{1}, \ldots, \alpha_{j-1}, \alpha_{j+1}, \ldots, \alpha_{2 n-1}} H_{\mathbb{C}_{\delta}}^{\alpha_{j}}\left(\alpha_{j+}\right) .
\end{aligned}
$$


In turn, by the explicit values in the proof of Lemma 4.7, this is given by

$$
\begin{gathered}
\frac{(-1)^{j+1}}{\sqrt{o_{j}}} F_{\Omega_{\delta}:\left\{e_{1}^{s_{1}, \ldots, e_{m}, a_{j}}, a_{j}^{\circ}\right\}}^{\alpha_{1}, \ldots, \alpha_{j-1}, \alpha_{j n-1}, \ldots \alpha_{2 n-1}}+(-1)^{j} F_{\Omega_{\delta}:\left\{e_{1}^{s_{1}}, \ldots, e_{m}^{s_{m}}\right\}}^{\alpha_{1}, \ldots, \alpha_{j-1}, \alpha_{j+1}, \ldots, \alpha_{2 n-1}} H_{\mathbb{C}_{\delta}}^{\alpha_{j}}\left(\alpha_{j+}\right) \\
=\frac{(-1)^{j+1}}{\sqrt{o_{j}}} F_{\Omega_{\delta}:\left\{e_{1}^{s_{1}}, \ldots, e_{m}^{s_{m}}\right\}}^{\alpha_{1}, \ldots, \alpha_{j-1}, \alpha_{j+1}, \ldots \alpha_{2 n-1}:\left[a_{j}\right]},
\end{gathered}
$$

using that $H_{\mathbb{C}_{\delta}}^{\alpha_{j}}\left(a_{j+}\right)=\frac{\mu+1}{2 \sqrt{o_{j}}}$ (see Remark 4.8). Identical computation gives the $\left[\Omega_{\delta}, 0\right]$ case, where now we use the fact that for every $a_{j}=\delta a_{j}^{\prime}$, we have $H_{\left[\mathbb{C}_{\delta}, 0\right]}^{\alpha_{j}}\left(\alpha_{j+}\right)=$ $\frac{\mu_{a_{j}^{\prime}}+1}{2 \sqrt{o_{j}}}$.

Remark 4.12. So far, we have assumed that the $2 n$ inputs $\alpha_{1}, \ldots, \alpha_{2 n}$ of the real observable are s-orientations of distinct $a_{1}, \ldots, a_{2 n}$. An important observation to be made is that the combinatorial definition of the real observable is robust enough for the pairwisefused case, where a medial vertex $e$ appears twice among the $2 n$ inputs ( as long as their respective orientations $o_{j}, o_{k}$ point to opposite directions.

In the complexified case, a medial vertex can appear twice, again oppositely oriented, among the first $2 n-1$ s-oriented medial vertices; if $a_{j}=a_{k}$ on $\Omega_{\delta}$ with opposite orientations $o_{j}$ and $o_{k}$, one can verify that the residue at $\alpha_{j}$ is given by

$$
\frac{(-1)^{j+1}}{\sqrt{o_{j}}} F_{\Omega_{\delta}:\left\{e_{1}^{s_{1}}, \ldots, e_{m}^{s_{m}}\right\}}^{\alpha_{1}, \ldots, \alpha_{j-1}, \alpha_{j+1}, \ldots, \alpha_{2 n-1}}-\frac{(-1)^{k+1}}{\sqrt{o_{k}}} F_{\Omega_{\delta}:\left\{e_{1}^{s_{1}}, \ldots, e_{m}^{s_{m}}\right\}}^{\alpha_{1}, \ldots, \alpha_{k-1}, \alpha_{k+1}, \ldots, \alpha_{2 n-1}} .
$$

This is precisely a directed superposition of the two residues derived in Lemma 4.7 for s-oriented medial vertices $\alpha_{j}, \alpha_{k}$; similarly Lemma 4.11 and the resulting Propositions 4.14-4.15, introduced in the next subsection, easily generalize to the pairwise-fused case.

We are now in position to connect the fused multipoint observables to multipoint Ising correlations.

Proposition 4.13. Suppose $\left\{b_{k}\right\}_{k=1}^{N}$ is a set of $N$ distinct interior edges in $\Omega_{\delta}$. We have

$$
\begin{array}{r}
\mathbb{E}_{\Omega_{\delta}}\left[\prod_{b \in\left\{b_{k}\right\}} \epsilon(b)\right]=(-1)^{N} 2^{N} F_{\Omega_{\delta}}^{\left[b_{1}, \ldots, b_{N}\right]}, \\
\frac{\mathbb{E}_{\Omega_{\delta}\left[\sigma_{0} \prod_{b \in\left\{b_{k}\right\}} \epsilon_{[0]}(b)\right]}}{\mathbb{E}_{\Omega_{\delta}}\left[\sigma_{0}\right]}=(-1)^{N} 2^{N} F_{\left[\Omega_{\delta}, 0\right]}^{\left[b_{1}, \ldots, b_{N}\right]} .
\end{array}
$$

Proof. The first identity was proved in [Hon10, Proposition 72] inductively, where our above extensions of the projection relations to adjacent edges now allow for the $b_{k}$ to be adjacent. Explicitly, denoting $\left\{e_{j}^{s_{j}}\right\}=\left\{e_{1}^{s_{1}}, \ldots, e_{m}^{s_{m}}\right\}$ for a set of edges (distinct from $b_{k}$ ) with inclusion variables $s_{j} \in\{\bullet, \circ\}$ and recalling $\mathcal{C}_{\Omega_{\delta}:\left\{e_{j}^{s}\right\}}$, it is straightforward to show by induction on $N$ (the base case is trivial),

$$
\mathbb{E}_{\Omega_{\delta}}\left[\mathbf{1}_{\left\{e_{j}^{s_{j}}\right\}} \prod_{b \in\left\{b_{k}\right\}} \epsilon(b)\right]=(-1)^{N} 2^{N} F_{\Omega_{\delta}:\left\{e_{j}^{s_{j}}\right\}}^{\left[b_{1}, \ldots, b_{N}\right]}
$$


(where for an edge $e$ separating faces $x, y$ with inclusion variable $s \in\{\bullet, \circ\}$, the indicator $\mathbf{1}_{\left\{e^{s}\right\}}$ denotes an indicator on the event that $\sigma_{x}=\sigma_{y}$ if $s=\circ$ and $\sigma_{x} \neq \sigma_{y}$ if $s=\bullet$ ) using the expansion

$$
\begin{aligned}
\mathbb{E}_{\Omega_{\delta}}\left[\mathbf{1}_{\left\{e_{j}^{s_{j}}\right\}} \epsilon\left(b_{1}\right) \cdots \epsilon\left(b_{N+1}\right)\right]= & (\mu-1) \mathbb{E}_{\Omega_{\delta}}\left[\mathbf{1}_{\left\{e_{j}^{s_{j}}, b_{N+1}^{\circ}\right\}} \epsilon\left(b_{1}\right) \cdots \epsilon\left(b_{N}\right)\right] \\
& +(\mu+1) \mathbb{E}_{\Omega_{\delta}}\left[\mathbf{1}_{\left\{e_{j}^{s_{j}}, b_{N+1}^{\bullet}\right.} \in\left(b_{1}\right) \cdots \epsilon\left(b_{N}\right)\right],
\end{aligned}
$$

and that by definition $F_{\Omega_{\delta}:\left\{e_{j}^{s_{j}}\right\}}^{\left[b_{1}, \ldots, b_{N}\right]}=F_{\Omega_{\delta}:\left\{e_{j}^{s_{j}}, b_{N+1}^{\circ}\right\}}^{\left[b_{1}, \ldots, b_{N}\right]}{ }^{s^{\prime}} F_{\Omega_{\delta}:\left\{e_{j}, b_{N+1}^{\bullet}\right.}^{\left[b_{1}, \ldots, b_{N}\right]}$

The second identity follows from an analogous process, where we note that for a collection of loops and walks $\omega$, we have $\sigma_{0}(\omega)=(-1)^{\ell(\omega)}$ due to the plus boundary condition.

4.3. Pfaffian formulae. Having related the Ising correlations to the fused observables, in this subsection we will elucidate how the recursive relation (4.3) gives rise to the Pfaffian relation in Sect. 4.4. The argument is identical to the one presented in Chapter 6 of [Hon10], albeit with the stronger lemmas introduced in Sect. 4.1 allowing the $\alpha_{i}$ 's to be adjacent.

We first prove that a $2 n$-point observable is in fact a Pfaffian of a matrix of two-point observables. Recall that for a $2 n \times 2 n$ antisymmetric matrix $A=\left(A_{j k}\right)_{j, k=1, \ldots, 2 n}$,

$$
\operatorname{Pf} A:=\frac{1}{2^{n} n !} \sum_{\sigma \in S^{2 n}} \operatorname{sgn}(\sigma) A_{\sigma(1) \sigma(2)} A_{\sigma(3) \sigma(4)} \cdots A_{\sigma(2 n-1) \sigma(2 n)},
$$

and we have the recursive expansion formula,

$$
\operatorname{Pf} A=\sum_{j=1}^{2 n-1}(-1)^{j} A_{j, 2 n} \operatorname{Pf} A_{\widehat{j ; 2 n}},
$$

where $A_{j ; 2 n}$ is the matrix where the $j$ and $2 n$-th rows and columns are removed.

Proposition 4.14. Suppose $\alpha_{j}=a_{j}^{o_{j}}, j=1, \ldots, 2 n$ are distinct (possibly pairwisefused) s-oriented interior medial vertices of $D_{\delta}=\Omega_{\delta}$ or $\left[\Omega_{\delta}, 0\right]$. Define the $2 n \times 2 n$ antisymmetric matrix

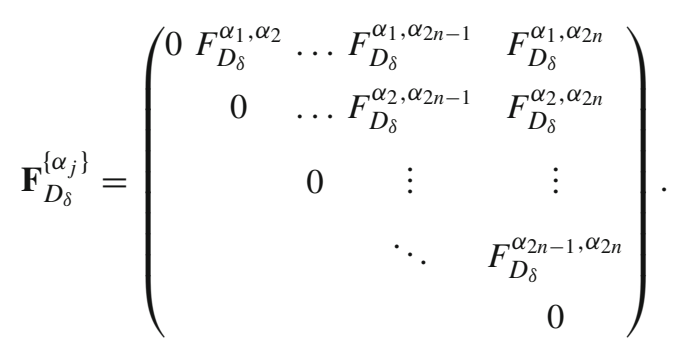

Then

$$
F_{D_{\delta}}^{\alpha_{1}, \ldots, \alpha_{2 n}}=\operatorname{Pf} \mathbf{F}_{D_{\delta}}^{\left\{\alpha_{j}\right\}} .
$$


Proof. The $2 \times 2$ case is trivial. Given the recursive formula (4.7) for the Pfaffian, inductively it suffices to show

$$
F_{D_{\delta}}^{\alpha_{1}, \ldots, \alpha_{2 n}}=\sum_{j=1}^{2 n-1}(-1)^{j} F_{D_{\delta}}^{\alpha_{1}, \ldots, \alpha_{j-1}, \alpha_{j+1} \ldots, \alpha_{2 n-1}} F_{D_{\delta}}^{\alpha_{j}, \alpha_{2 n}} .
$$

The strategy is to use the boundary value problem uniqueness result (Lemma 2.11) to show that the function $H_{D_{\delta}}^{\alpha_{1}, \ldots, \alpha_{2 n-1}}\left(a_{2 n}\right)-\sum_{j=1}^{2 n-1}(-1)^{j} F_{D_{\delta}}^{\alpha_{1}, \ldots, \alpha_{j-1}, \alpha_{j+1} \ldots, \alpha_{2 n-1}} H_{D_{\delta}}^{\alpha_{j}}\left(a_{2 n}\right)$ is identically zero. The boundary condition is obviously satisfied; the fact that all singularities at $\alpha_{1}, \ldots, \alpha_{2 n-1}$ are removable, i.e. have residue zero, is immediate from Lemma 4.7.

We now extend the Pfaffian representation to the fused observables. For any edge $e$, write $e^{+}=e^{o^{+}}, e^{-}=e^{o^{-}}$for a pair of s-orientations $o^{ \pm}$of $e$, so that $o^{+}=e^{\pi i} o^{-}$. Recall the use of the $\dagger$ to denote

$$
F_{\Omega_{\delta}}^{\dagger}=F_{\Omega_{\delta}}-F_{\mathbb{C}_{\delta}} \quad \text { and } \quad F_{\left[\Omega_{\delta}, 0\right]}^{\dagger}=F_{\left[\Omega_{\delta}, 0\right]}-F_{\left[\mathbb{C}_{\delta}, 0\right]} .
$$

For edges $e_{1}, \ldots, e_{m}$ define the $2 m \times 2 m$ antisymmetric matrix with $F_{D_{\delta}}^{\dagger e_{i}^{+}, e_{i}^{-}}$on the anti-diagonal $i+j=2 m+1, i \leq m$, and more generally, entries,

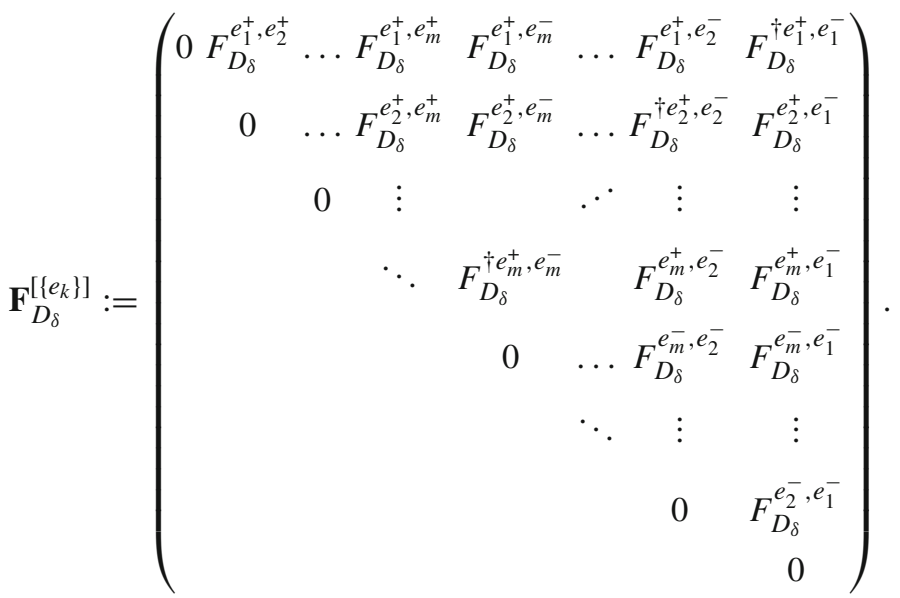

Proposition 4.15. Suppose $\alpha_{j}=a_{j}^{o_{j}}$ for $j=1, \ldots, 2 n$ and $e_{k}$ for $k=1, \ldots, m$ are distinct (possibly pairwise-fused) interior medial vertices of $D_{\delta}=\Omega_{\delta}$ or $\left[\Omega_{\delta}, 0\right]$. Define the block antisymmetric $2(m+n) \times 2(m+n)$ matrix $F_{D_{\delta}}^{\left\{a_{j}\right\}:\left[\left\{e_{k}\right\}\right]}$ by

$$
\begin{aligned}
& \mathbf{F}_{D_{\delta}}^{\left\{a_{j}\right\}:\left[\left\{e_{k}\right\}\right]}:=\left(\begin{array}{cc}
\mathbf{F}_{D_{\delta}}^{\left[\left\{e_{k}\right\}\right]} & -\left[\mathbf{F}_{D_{\delta}}^{\left\{a_{j}\right\} \times\left[\left\{e_{k}\right\}\right]}\right]^{T} \\
\mathbf{F}_{D_{\delta}}^{\left\{a_{j}\right\} \times\left[\left\{e_{k}\right\}\right]} & \mathbf{F}_{D_{\delta}}^{\left\{a_{j}\right\}}
\end{array}\right) \text {, where } \\
& \mathbf{F}_{D_{\delta}}^{\left\{a_{j}\right\} \times\left[\left\{e_{k}\right\}\right]}:=\left(\begin{array}{cccccc}
F_{D_{\delta}}^{\alpha_{1}, e_{1}^{+}} & \ldots & F_{D_{\delta}}^{\alpha_{1}, e_{m}^{+}} & F_{D_{\delta}}^{\alpha_{1}, e_{m}^{-}} & \ldots & F_{D_{\delta}}^{\alpha_{1}, e_{1}^{-}} \\
\vdots & & \vdots & \vdots & & \vdots \\
F_{D_{\delta}}^{\alpha_{2 n}, e_{1}^{+}} & \ldots & F_{D_{\delta}}^{\alpha_{2 n}, e_{m}^{+}} & F_{D_{\delta}}^{\alpha_{2 n}, e_{m}^{-}} & \ldots & F_{D_{\delta}, e_{1}^{-}}^{\alpha_{2 n}}
\end{array}\right) .
\end{aligned}
$$


Then

$$
F_{D_{\delta}}^{\alpha_{1}, \ldots, \alpha_{2 n}:\left[e_{1}, \ldots, e_{m}\right]}=\operatorname{Pf} \mathbf{F}_{D_{\delta}}^{\left\{a_{j}\right\}:\left[\left\{e_{k}\right\}\right]} .
$$

In particular, since $F_{D_{\delta}}^{\alpha_{1}, \ldots, \alpha_{2 n}:\left[e_{1}, \ldots, e_{m}\right]}$ does not depend on the choice of s-orientations $o_{j}^{+}, o_{j}^{-}$on $e_{k}$, the Pfaffian does not.

Proof. Without loss of generality, we will assume $D_{\delta}=\Omega_{\delta}$; the case $D_{\delta}=\left[\Omega_{\delta}, 0\right]$ can be treated identically. We use induction on $m$. The case $m=0$ is given by Proposition 4.14. Now we assume the result holds for $m$, and consider the case $m+1$. By Lemma 4.11, if $e_{m+1}^{+}=e_{m+1}^{o}$ is an s-orientation of $e_{m+1}$, we can extend to the removed singularity

$$
\left[H_{\Omega_{\delta}}^{\alpha_{1}, \ldots, \alpha_{2 n}, e_{m+1}^{+}:\left[e_{1}, \ldots, e_{m}\right]}-F_{\Omega_{\delta}}^{\alpha_{1}, \ldots, \alpha_{2 n}:\left[e_{1}, \ldots, e_{m}\right]} H_{\mathbb{C}_{\delta}}^{e_{m+1}^{+}}\right]\left(e_{m+1}\right):=\frac{1}{\sqrt{o}} F_{\Omega_{\delta}}^{\alpha_{1}, \ldots \alpha_{2 n}:\left[e_{1}, \ldots, e_{m+1}\right]}
$$

Then by the projection relations given by s-holomorphicity, we can deduce

$$
F_{\Omega_{\delta}}^{\alpha_{1}, \ldots, \alpha_{2 n}, e_{m+1}^{+}, e_{m+1}^{-}:\left[e_{1}, \ldots, e_{m}\right]}-F_{\Omega_{\delta}}^{\alpha_{1}, \ldots, \alpha_{2 n}:\left[e_{1}, \ldots, e_{m}\right]} F_{\mathbb{C}_{\delta}}^{e_{m+1}^{+}, e_{m+1}^{-}}:=F_{\Omega_{\delta}}^{\alpha_{1}, \ldots \alpha_{2 n}:\left[e_{1}, \ldots, e_{m+1}\right]}
$$

By the inductive hypothesis, we have the desired Pfaffian formulations of $F_{\Omega_{\delta}}^{\alpha_{1}, \ldots, \alpha_{2 n}, e_{m+1}^{+}, e_{m+1}^{-}:\left[e_{1}, \ldots, e_{m}\right]}$ and $F_{\Omega_{\delta}}^{\alpha_{1}, \ldots, \alpha_{2 n}:\left[e_{1}, \ldots, e_{m}\right]}$. Expanding the Pfaffian along the last column as in (4.7) the result easily follows.

4.4. Observables and Ising correlations. In this subsection we present the connection between Ising model correlations and spin-weighted correlations and the two-point observables defined in Sect.3.1. These formulae follow immediately from the results of Sects. 4.2 and 4.3.

4.4.1. Spin-symmetric correlations Recall that we have defined a spin-symmetric correlation as the expectation of a product of energy densities which scale with the mesh size $\delta$. Our characterization of the correlation consists of a Pfaffian involving the real observables introduced in Sect. 3. For an edge $e$, we will denote by $e^{+}:=e^{o^{+}}, e^{-}:=e^{o^{-}}$for any pair of s-orientations $o^{+}, o^{-}$such that $o^{+}=e^{\pi i} o^{-}$; the following characterization shows in particular that the Pfaffian does not depend on such choice.

Proposition 4.16. For any collection of distinct (possibly adjacent) interior edges $e_{1}, \ldots, e_{m}$ in $\Omega_{\delta}$,

$$
\mathbb{E}_{\Omega_{\delta}}\left[\prod_{e \in\left\{e_{k}\right\}} \epsilon(e)\right]=(-1)^{m} 2^{m} \operatorname{Pf}\left(\mathbf{F}_{\Omega_{\delta}}^{\left[\left\{e_{k}\right\}\right]}\right),
$$

where for any admissible s-orientations, $o_{1}^{ \pm}, \ldots, o_{m}^{ \pm}$, the antisymmetric matrix $\mathbf{F}_{\Omega_{\delta}}^{\left[\left\{e_{k}\right\}\right]}$ is given by 


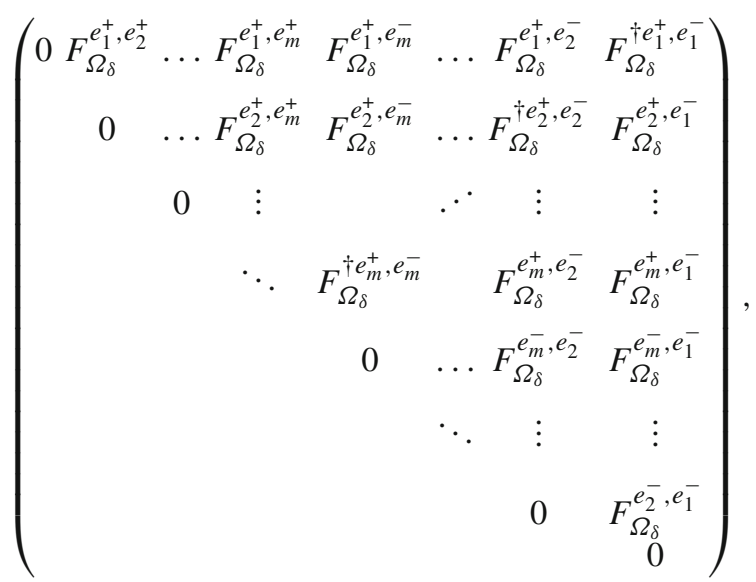

where $F_{\Omega_{\delta}}^{\dagger \alpha, \zeta}:=F_{\Omega_{\delta}}^{\alpha, \zeta}-F_{\mathbb{C}_{\delta}}^{\alpha, \zeta}$.

Proof. Starting from the characterization of Proposition 4.13, we can calculate the fused observable via an application of Proposition 4.15.

4.4.2. Spin-antisymmetric correlations Recall that a spin-weighted correlation was defined as the expectation of a product of the spin at 0 and energy densities on adjacent sites. We characterize it as a Pfaffian analogously to the previous subsection, but with $\Omega_{\delta}$ replaced by its double cover and the two-point fermion replaced with the spin-fermion observable. Accordingly, we again fix orientations $e^{+}, e^{-}$as well as a choice of lift in $\left[\Omega_{\delta}, 0\right]$ for an edge $e$ in $\Omega_{\delta}$, on which the value of the Pfaffian does not depend.

Proposition 4.17. For any collection of distinct (possibly adjacent) interior edges $e_{1}, \ldots, e_{m}$ in $\Omega_{\delta}$,

$$
\frac{1}{\mathbb{E}_{\Omega_{\delta}}\left[\sigma_{0}\right]} \mathbb{E}_{\Omega_{\delta}}\left[\sigma_{0} \prod_{e \in\left\{e_{k}\right\}} \epsilon_{[0]}(e)\right]=(-1)^{m} 2^{m} \operatorname{Pf}\left(\mathbf{F}_{\left[\Omega_{\delta}, 0\right]}^{\left[\left\{e_{k}\right\}\right]}\right),
$$

where for any admissible s-orientations $o_{1}^{ \pm}, \ldots, o_{m}^{ \pm}$, the antisymmetric matrix $\mathbf{F}_{\left[\Omega_{\delta}, 0\right]}^{\left[\left\{e_{k}\right\}\right]}$ is given by

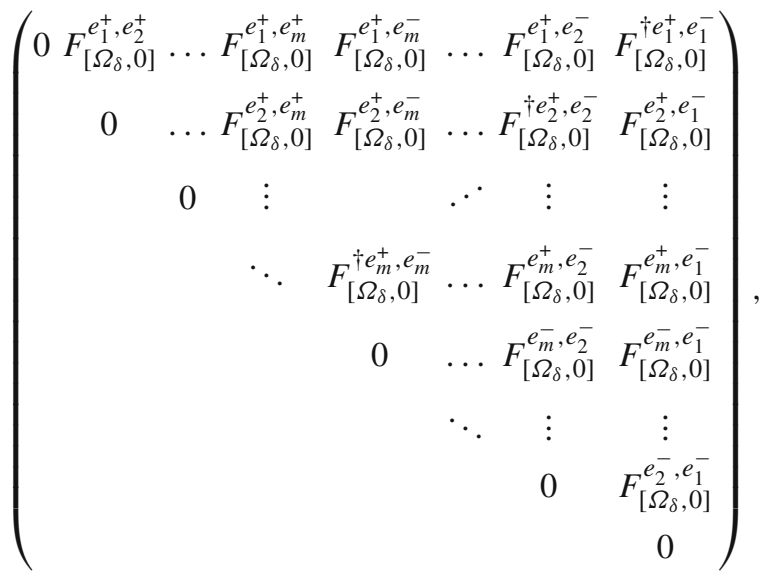


where $F_{\left[\Omega_{\delta}, 0\right]}^{\dagger \alpha, \zeta}:=F_{\left[\Omega_{\delta}, 0\right]}^{\alpha, \zeta}-F_{\left[\mathbb{C}_{\delta}, 0\right]}^{\alpha, \zeta}$.

Proof. Again starting from the characterization of Proposition 4.13, we can calculate the fused observable via Proposition 4.15, using now the equations corresponding to the spin-fermion.

\section{Scaling Limits of Observables}

We have thus far defined the discrete observables which encode probabilistic information in the form of $n$-point correlations. As a result of the Pfaffian formulae of Sect. 4.4, it suffices to consider two-point discrete observables since all multipoint correlations can now be written in terms of only two-point observables. In this section, we introduce the continuous observables, which are precisely defined to have continuous analogues of the properties satisfied by the discrete observables; see Sect. 2. With the appropriate scaling, the discrete observables will be shown to converge to these continuous observables.

As the heart of convergence proofs is the extension of the convergence results of [CHI15] for $H_{\left[\Omega_{\delta}, 0\right]}^{\alpha}$ to the case where the singularity point $\alpha$ is no longer at $\alpha_{0}$, familiarity with the proofs of convergence in [CHI15] for the case $\alpha=\alpha_{0}$ is very helpful to understanding the sequel.

5.1. Integration of the square. Write $v_{z}$ for the unit outward normal vector at $z \in \partial \Omega$. We have the following characterization of the complex fermion observable $H_{\Omega_{\delta}}$ in terms of discrete complex analysis:

Proposition 5.1 (see [Hon10]). Let $\alpha=a^{o}$ be an s-oriented medial vertex of $\Omega_{\delta}$ that is not on the boundary. The function $H_{\Omega_{\delta}}^{\alpha}$ is the unique function such that:

- $H_{\Omega_{\delta}}^{\alpha}$ is s-holomorphic on $\Omega_{\delta} \backslash\{a\}$;

- $H_{\Omega_{\delta}}^{\alpha}$ has discrete residue 1 at $\alpha: H_{\Omega_{\delta}}^{\alpha}\left(\alpha_{+}\right)-H_{\Omega_{\delta}}^{\alpha}\left(\alpha_{-}\right)=\frac{1}{\sqrt{o}}$;

- $H_{\Omega_{\delta}}^{\alpha}(z) \sqrt{\nu_{z}} \in \mathbb{R}$ for any boundary medial vertex $z$.

Similarly, we have the following characterization of the complex spin-fermion observable $H_{\left[\Omega_{\delta} 0\right]}$.

Proposition 5.2 Let $\alpha=a^{o}$ be an s-oriented medial vertex of $\left[\Omega_{\delta}, 0\right]$ that is not on the boundary. The function $H_{\left[\Omega_{\delta}, 0\right]}^{\alpha}$ is the unique function such that:

- $H_{\left[\Omega_{\delta}, 0\right]}^{\alpha}$ has monodromy -1 around 0 ;

- $H_{\left[\Omega_{\delta}, 0\right]}^{\alpha}$ is s-holomorphic on $\left[\Omega_{\delta}, 0\right] \backslash\left\{a, a^{*}\right\}$, where $a, a^{*}$ are on opposite sheets of $\left[\Omega_{\delta}, 0\right]$;

- $H_{\left[\Omega_{\delta}, 0\right]}^{\alpha}$ has discrete residue 1 at $\alpha: H_{\left[\Omega_{\delta}, 0\right]}^{\alpha}\left(\alpha_{+}\right)-H_{\left[\Omega_{\delta}, 0\right]}^{\alpha}\left(\alpha_{-}\right)=\frac{1}{\sqrt{o}}$;

- $H_{\left[\Omega_{\delta}, 0\right]}^{\alpha}(z) \sqrt{\nu_{z}} \in \mathbb{R}$ for any boundary medial vertex $z$.

Proof of Propositions 5.1-5.2. Monodromy for the latter is clear from the sheet factor $\mathrm{s}_{\alpha, \zeta}$. Proposition 4.5 and Lemmas 4.7 and 4.9 provide the remaining properties. Uniqueness follows from Lemma 2.11.

We now consider the square integral $Q_{\delta}^{\alpha}$ of the observables. These are the same discrete square integral analogues $\mathbb{I}_{\delta}\left(H_{\delta}\right)=\operatorname{Re} \int\left(H_{\delta}\right)^{2}$ introduced in Sect. 2.3 in the case where $H_{\delta}$ is one of $H_{\Omega_{\delta}}^{\alpha}, H_{\left[\Omega_{\delta}, 0\right]}^{\alpha}$, but we need to analyze their properties near the singularity at 
$\alpha$ and the monodromy. These square integrals will be our primary means of estimating both observables, but for conciseness, we will assume that we are working with $H_{\left[\Omega_{\delta}, 0\right]}^{\alpha}$. Neither the construction nor the properties are changed in the $H_{\Omega_{\delta}}^{\alpha}$ case, except for the fact that there are no longer complications arising from the branching at 0 .

Proposition 5.3. Suppose $\alpha=a^{o}$ is an s-oriented corner in $\left[\Omega_{\delta}, 0\right]$. Consider the singlevalued integral of the square $Q_{\delta}^{\alpha}:=\mathbb{I}_{\delta}\left[H_{\left[\Omega_{\delta}, 0\right]}^{\alpha}\right]: \mathcal{F}_{\Omega_{\delta}} \cup \mathcal{V}_{\Omega_{\delta}} \rightarrow \mathbb{R}$ constructed with the usual rule

$$
Q_{\delta}^{\alpha}(w)-Q_{\delta}^{\alpha}(v)=2 \delta\left|H_{\left[\Omega_{\delta}, 0\right]}^{\alpha}\left(\frac{1}{2}(w+v)\right)\right|^{2}
$$

where $w$ is a face, $v$ is a vertex incident to the face, so that $\frac{1}{2}(w+v)$ is the corner between them (note that at the singularity $a,\left|H_{\left[\Omega_{\delta}, 0\right]}^{\alpha}(a)\right|^{2}=\frac{1}{8}$ ), and the Dirichlet boundary condition

$$
Q_{\delta}^{\alpha}(w)=0 \text { for } w \in \partial \mathcal{F}_{\Omega_{\delta}} .
$$

It has

- $\Delta_{\delta} Q_{\delta}^{\alpha}=2 \delta\left|\partial_{\delta} H_{\left[\Omega_{\delta}, 0\right]}^{\alpha}\right|^{2}$ on $\mathcal{F}_{\Omega_{\delta}} \backslash\left\{0, a-o \frac{\delta}{2}\right\}, \Delta_{\delta} Q_{\delta}^{\alpha}=-2 \delta\left|\partial_{\delta} H_{\left[\Omega_{\delta}, 0\right]}^{\alpha}\right|^{2}$ on $\mathcal{V}_{\Omega_{\delta}} \backslash\left\{a+o \frac{\delta}{2}\right\}$;

- The outer normal derivative $\partial_{\text {out }} Q_{\Omega}^{\alpha}=\sqrt{2}\left|H_{\left[\Omega_{\delta}, 0\right]}^{\alpha}\right|^{2}$ on $\partial \mathcal{V}_{\Omega_{\delta}}^{m}$.

Proof. The proof follows from direct computations in Chapter 2 of [Hon10] and Section 3.3 of [ChSm12]. Note that as in [CHI15, Proposition 3.6], the singularity at $a$ (two projections from neighboring medial vertices differing only by a sign) and branching at 0 does not affect well-definedness of $Q_{\delta}^{\alpha}$, but does affect the Laplacian at $0, a \pm o \frac{\delta}{2}$.

Remark 5.4. In keeping with the Dirichlet boundary condition, we can define $\tilde{Q}_{\delta}^{\alpha}$ which simply modifies the value of $Q_{\delta}^{\alpha}$ on $\partial \mathcal{V}_{\Omega_{\delta}}$ to be zero. This affects the Laplacian and normal derivative, but one can define an alternate Laplacian $\tilde{\Delta}_{\delta}$ modified at the boundary which gives $\tilde{\Delta}_{\delta} \tilde{Q}_{\delta}^{\alpha}=\Delta_{\delta} Q_{\delta}^{\alpha}$; see [CHI15, Preposition 3.6].

Remark 5.5. Similarly, one can define the integral $Q_{\delta}^{\dagger \alpha}:=\mathbb{I}_{\delta}\left[H_{\left[\Omega_{\delta}, 0\right]}^{\alpha}-H_{\left[\mathbb{C}_{\delta}, 0\right]}^{\alpha}\right]$; this has the advantage of removing the singularity at $\alpha$, so that we have sub- and superharmonicity at all points except for 0 . [CHI15, Remark 3.8] notes that in the special case $a=\frac{\delta}{2}$ we in fact do have sub-harmonicity at $a$, owing to the fact that $\left[H_{\left[\Omega_{\delta}, 0\right]}^{\alpha}-H_{\left[\mathbb{C}_{\delta}, 0\right]}^{\alpha}\right](a):=0$.

5.2. Continuous observables. The continuous observables are the solutions of the continuous Riemann boundary value problem corresponding to the discrete b.v.p. of Lemma 2.11. We prove that they are in fact the scaling limits of their discrete counterparts in Sect. 5.3. 
5.2.1. Continuous full-plane observables We begin by defining the continuous fullplane observables. In analogy with the discrete case, we define an s-orientation $o$ of a point $a$ in a continuous domain $\Omega$ as a choice of any unit complex number $o$ with a specified square root.

Definition 5.6. Let $\alpha:=a^{o}$ and $\zeta:=z^{p}$ be two s-oriented points of $\mathbb{C}$ with $a \neq z$. We define the continuous fermion observable $f_{\mathbb{C}}$ by

$$
f_{\mathbb{C}}^{\alpha}(\zeta):=i \sqrt{p} \cdot \mathrm{P}_{\frac{1}{i \sqrt{p}} \mathbb{R}}\left[h_{\mathbb{C}}^{\alpha}(z)\right]=\operatorname{Re}\left[i \sqrt{p} \cdot h_{\mathbb{C}}^{\alpha}(z)\right]
$$

where the complex observable $h_{\mathbb{C}}$ is defined by

$$
h_{\mathbb{C}}^{\alpha}(z):=\frac{1}{\sqrt{2} \pi} \frac{\sqrt{o}}{z-a} .
$$

Analogously to the previous definition, we proceed to define the continuous full-plane spin-fermion observable. Since the source point $\delta \alpha$ of the discrete two-point spin-fermion tends to the monodromy point 0 as $\delta \rightarrow 0$, we fix it as $\alpha \in \mathcal{V}_{\left[\mathbb{C}_{1}, 0\right]}^{c m}$, and formally denote the dependence of the continuous spin-fermion on $\alpha$ by $d \alpha$ (not to be confused with $\delta \alpha \in \mathcal{V}_{\left[\mathbb{C}_{\delta}, 0\right]}^{c m}$, which is the scaling of $\alpha$ by $\delta>0$ ).

Definition 5.7. Let $\alpha:=a^{o}$ be an s-oriented corner or medial vertex of $\left[\mathbb{C}_{1}, 0\right]$. Let $\zeta:=$ $z^{p}$ be an s-oriented point of $[\mathbb{C}, 0]$. We define the continuous spin-fermion observable $f_{[\mathbb{C}, 0]}$ by

$$
f_{[\mathbb{C}, 0]}^{d \alpha}(\zeta):=\operatorname{Re}\left[i \sqrt{p} \cdot h_{[\mathbb{C}, 0]}^{d \alpha}(z)\right]
$$

where the complex observable $h_{[\mathbb{C}, 0]}^{d \alpha}$ is defined by

$$
h_{[\mathbb{C}, 0]}^{d \alpha}(z):=\frac{C_{\alpha}}{\sqrt{z}},
$$

and the scaling limit factor $C_{\alpha} \in \mathbb{R}$ is given by

$$
C_{\alpha}=-\operatorname{Re}\left[i \sqrt{o}\left(\tilde{G}_{\left[\mathbb{C}_{1}, 0\right]}^{+}-\tilde{G}_{\left[\mathbb{C}_{1}, 0\right]}^{-}\right)(a)\right] .
$$

Recall, here, that $\tilde{G}^{ \pm}$are the discrete analogs of $\frac{i}{2 \sqrt{2}} \sqrt{z}$ given by Definition 3.25 . Note that when $\alpha$ is an s-oriented corner, we do not need to take the real-part in the definition above as $i \sqrt{o} \tilde{G}^{ \pm}(a)$ are already real, due to s-holomorphicity. In particular, if $\alpha$ is an s-oriented real or imaginary corner we have that

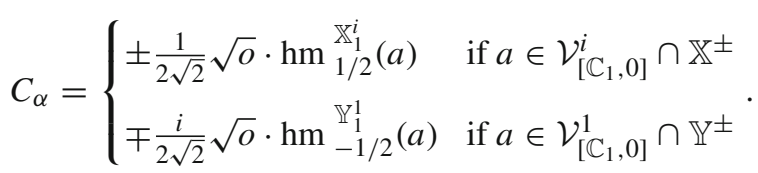


5.2.2. Continuous bounded domain observables Let $\Omega \subset \mathbb{C}$ be a bounded simply connected domain containing 0 . Recall that we denote by $\varphi$ the conformal mapping from $\Omega$ to the open unit disk $\mathbb{D}$ with $\varphi(0)=z$ and $\varphi^{\prime}(0)>0$. Here we set $z=0$. We transform $\alpha=a^{o}$ as $\varphi(\alpha)=\varphi(a)^{o^{\prime}}, o^{\prime}=\left(\sqrt{\varphi^{\prime}(a)} \sqrt{o}\right)^{2} /\left|\varphi^{\prime}(a)\right|$ under $\varphi$ (with a continuous square root branch choice for $\varphi^{\prime}$ such that $\left.\sqrt{\varphi^{\prime}(0)}>0\right)$.

Definition 5.8. Let $\alpha=a^{o}, \zeta=z^{p}$ be two s-oriented points of $\Omega$ with $a \neq z$. We define the fermion observable $f_{\Omega}$ by

$$
f_{\Omega}^{\alpha}(\zeta):=i \sqrt{p} \mathrm{P}_{\frac{1}{i \sqrt{p}} \mathbb{R}}\left[h_{\Omega}^{\alpha}(z)\right]=\operatorname{Re}\left[i \sqrt{p} \cdot h_{\Omega}^{\alpha}(z)\right],
$$

where the complexified fermion observable $h_{\Omega}$ is defined by

$$
\begin{aligned}
h_{\Omega}^{\alpha}(z) & :=\sqrt{\left|\varphi^{\prime}(a)\right|} \sqrt{\varphi^{\prime}(z)} h_{\mathbb{D}}^{\varphi(\alpha)}(\varphi(z)), \text { where } \\
h_{\mathbb{D}}^{\alpha}(z) & :=\frac{1}{\sqrt{2} \pi}\left(\frac{1}{\sqrt{o}} \cdot \frac{1}{1-\bar{a} z}+\frac{\sqrt{o}}{z-a}\right) .
\end{aligned}
$$

Definition 5.9. Let $\alpha=a^{o}$ be an s-oriented corner or medial vertex on $\left[\mathbb{C}_{1}, 0\right]$, and $\zeta=z^{p}$ be an s-oriented point of $\Omega$ with $a \neq z$. We define the spin-fermion observable $f_{[\Omega, 0]}$ by

$$
f_{[\Omega, 0]}^{d \alpha}(\zeta):=i \sqrt{p} \mathrm{P}_{\frac{1}{i \sqrt{p}}} \mathbb{R}\left[h_{[\Omega, 0]}^{d \alpha}(z)\right]=\operatorname{Re}\left[i \sqrt{p} \cdot h_{[\Omega, 0]}^{d \alpha}(z)\right],
$$

where the complexified spin-fermion observable $h_{[\Omega, 0]}$ is defined by

$$
h_{[\Omega, 0]}^{d \alpha}(z):=\sqrt{\varphi^{\prime}(z)} h_{\mathbb{D}}^{d \alpha}(\varphi(z)), \quad \text { where } \quad h_{[\mathbb{D}, 0]}^{d \alpha}(z)=\frac{C_{\alpha}}{\sqrt{z}} .
$$

Remark 5.10. As explained above, these definitions imply continuous versions of the properties satisfied by the discrete observables; they share the singularities of the fullplane observables, and satisfy the boundary condition $h_{\Omega}^{\alpha}(z), h_{[\Omega, 0]}^{d \alpha}(z) \in v_{z}{ }^{-1 / 2} \mathbb{R}$, if $v_{z}$ is the unit outward normal vector at $z \in \partial \Omega$. These also uniquely characterize a holomorphic function: see [Hon10, Proposition 48] and [CHI15, Lemma 2.9].

5.3. Observable convergence: statements. We now state the two observable convergence results for the fermion and spin-fermion that are needed for the proof of the main theorem in Sect. 6. The bulk of the remaining work is in the proof of the spin-fermion observable convergence; that proof is deferred until the next subsection.

In what follows, we say an s-holomorphic function $H_{\delta}: \mathcal{V}_{D_{\delta}}^{c m} \rightarrow \mathbb{C}$ converges to a continuous function $h: D \rightarrow \mathbb{C}$ if for any sequence $a_{\delta} \in \mathcal{V}_{D_{\delta}}^{m}$ such that $a_{\delta} \rightarrow a \in D$ we have $H_{\delta}\left(a_{\delta}\right) \rightarrow h(a)$. Equivalently, the values of $H_{\delta}$ on type 1 and $i$ corners respectively converge to the real and imaginary parts of $h$.

For notational convenience and concreteness, when we take $z \in D$ to be the argument of an s-holomorphic function $H_{\delta}$ defined on $\mathcal{V}_{D_{\delta}}^{c m}$, we will take a closest medial vertex $z_{\delta} \in \mathcal{V}_{D_{\delta}}^{m}$ to $z$ and evaluate it at $z_{\delta}$. Then the convergence is often uniform on a compact set $K \subset D$, i.e. $\left|H_{\delta}\left(z_{\delta}\right)-h(z)\right|$ is small uniformly in $z \in K$.

By a slight abuse of notation, we will then use the notation $\alpha=a^{o}$ and $\zeta=z^{p}$ both for the the s-orientations of $a$ or $z$, and those of $a_{\delta}$ and $z \delta$. 
Theorem 5.11 ([Hon10, Theorem 91]). As $\delta \rightarrow 0$, we have

$$
\frac{1}{\delta}\left(F_{\Omega_{\delta}}^{\alpha}-F_{\mathbb{C}_{\delta}}^{\alpha}\right)(\zeta) \rightarrow\left(f_{\Omega}^{\alpha}-f_{\mathbb{C}}^{\alpha}\right)(\zeta)
$$

uniformly for $a, z$ away from $\partial \Omega$.

Now let $\alpha:=a^{o}$ and $\zeta:=z^{p}$ be s-oriented corners on $\left[\mathbb{C}_{1}, 0\right]$. For $\delta>0$, denote by $\delta \alpha:=(\delta a)^{o}$ and $\delta \zeta:=(\delta z)^{p}$ their corresponding scaled versions on $\left[\mathbb{C}_{\delta}, 0\right]$ and, for sufficiently small $\delta$, on $\left[\Omega_{\delta}, 0\right]$. Recall that $G, \tilde{G}^{ \pm}$are the discrete analogues of $\frac{1}{2 \sqrt{2}} \sqrt{z}, \frac{i}{2 \sqrt{2}} \sqrt{z}$, respectively, introduced in Definition 3.25.

Theorem 5.12 For $\alpha, \zeta$ any s-oriented corners or medial vertices in $\left[\mathbb{C}_{1}, 0\right]$, we have as $\delta \rightarrow 0$,

$$
\begin{aligned}
& \frac{1}{\delta}\left(F_{\left[\Omega_{\delta}, 0\right]}^{\delta \alpha}-F_{\left[\mathbb{C}_{\delta}, 0\right]}^{\delta \alpha}\right)(\delta \zeta) \\
& \quad \rightarrow 2 \cdot 2 \sqrt{2} \operatorname{Re} \mathcal{A}_{\Omega} \cdot\left(C_{\alpha} \operatorname{Re}\left[i \sqrt{p} G_{\left[\mathbb{C}_{1}, 0\right]}(z)\right]-C_{\zeta} \operatorname{Re}\left[i \sqrt{o} G_{\left[\mathbb{C}_{1}, 0\right]}(a)\right]\right) \\
& \quad+2 \cdot 2 \sqrt{2} \operatorname{Im} \mathcal{A}_{\Omega} \cdot\left(C_{\alpha} \operatorname{Re}\left[i \sqrt{p} \tilde{G}_{\left[\mathbb{C}_{1}, 0\right]}^{-}(z)\right]-C_{\zeta} \operatorname{Re}\left[i \sqrt{o} \tilde{G}_{\left[\mathbb{C}_{1}, 0\right]}^{-}(a)\right]\right),
\end{aligned}
$$

where, for $\alpha_{0}=\frac{1}{2}^{o}$ as before, $\mathcal{A}_{\Omega}$ is the coefficient in the expansion

$$
h_{\Omega}^{d \alpha_{0}}(z)=\frac{1}{2 \sqrt{2}}\left(\frac{1}{\sqrt{z}}+2 \mathcal{A}_{\Omega} \sqrt{z}+O\left(|z|^{3 / 2}\right)\right) .
$$

Proof. This statement is the consequence of results proved in Sect. 5.4. Theorem 5.18 shows the following in the case where $\alpha, \zeta$ are both s-oriented corners: $\frac{1}{\delta}\left[H_{\left[\Omega_{\delta}, 0\right]}^{\delta \alpha}(\delta z)-\right.$ $\left.H_{\left[\mathbb{C}_{\delta}, 0\right]}^{\delta \alpha}(\delta z)\right]$ converges to

$$
\begin{aligned}
& 2 \cdot 2 \sqrt{2} \cdot\left[\operatorname{Re} \mathcal{A}_{\Omega} \cdot\left(C_{\alpha} G_{\left[\mathbb{C}_{1}, 0\right]}(z)+i \sqrt{o}\left[\tilde{G}_{\left[\mathbb{C}_{1}, 0\right]}^{+}-\tilde{G}_{\left[\mathbb{C}_{1}, 0\right]}^{-}\right](z) G_{\left[\mathbb{C}_{1}, 0\right]}(a)\right)\right. \\
& \left.+\operatorname{Im} \mathcal{A}_{\Omega} \cdot\left(C_{\alpha} \tilde{G}_{\left[\mathbb{C}_{1}, 0\right]}^{-}(z)+i \sqrt{o}\left[\tilde{G}_{\left[\mathbb{C}_{1}, 0\right]}^{+}-\tilde{G}_{\left[\mathbb{C}_{1}, 0\right]}^{-}\right](z) \tilde{G}_{\left[\mathbb{C}_{1}, 0\right]}^{-}(a)\right)\right],
\end{aligned}
$$

where we used Corollary 5.19 in order to identify $\tilde{C}_{\alpha}$ with $C_{\alpha}$ defined by (5.1). This formula also applies in the case where $z$ is a medial vertex by linearity. Reformulation in the form of the theorem statement follows from the definition of the real observable; we can then swap $\alpha, \zeta$ from the antisymmetry of the real observable and proceed to prove the result for the case where both are medial vertices.

Remark 5.13. It was shown in [CHI15, Lemma 2.21] that $\mathcal{A}_{\Omega}=\mathcal{A}_{[\Omega, 0]}=$ $-\left.\frac{1}{4} \partial_{z} \log r_{\Omega}(z)\right|_{z=0}$ which is the logarithmic derivative of the conformal radius as viewed from $0 \in \Omega$. This is used in Theorem 6.6 to identify the coefficient in the first-order correction to spin weighted correlations with $-\left.\frac{1}{4} \partial_{z} \log r_{\Omega}(z)\right|_{z=0}$.

5.4. Observable convergence: proofs. We now prove the aforementioned observable convergence theorems, in particular, the convergence of the full-plane observables and Theorem 5.12.

A tricky point of this subsection is that the explicit coefficient $C_{\alpha}$ of Definition 5.7, in the claimed limit $h_{[\mathbb{C}, 0]}^{d \alpha}(z)=C_{\alpha} / \sqrt{z}$ of the full-plane spinor $H_{\left[\mathbb{C}_{\delta}, 0\right]}^{\delta \alpha}$, can only be identified as such once all convergence results are proven; until then we introduce a recursively defined stand-in $\tilde{C}_{\alpha}$ and $\tilde{h}_{[\mathbb{C}, 0]}^{d \alpha}(z):=\tilde{C}_{\alpha} / \sqrt{z}$. We then show the equality $\tilde{C}_{\alpha}=C_{\alpha}$ at the end of this subsection in Corollary 5.19. 
5.4.1. Convergence of the full-plane observables We first state direct extensions of some results in [CHI15] regarding convergence of the full-plane observables and functions. In [CHI15], the renormalization factor $\vartheta(\delta):=\mathrm{hm}_{0}^{\mathbb{C}_{\delta} \backslash \mathbb{R}_{<0}}\left(2 \delta\left\lfloor(2 \delta)^{-1}\right\rfloor\right)$ is used; we are able to calculate the constant explicitly thanks to Proposition A.1. Specifically, writing $N:=\left\lfloor(2 \delta)^{-1}\right\rfloor, \vartheta(\delta)=\frac{1}{2} \cdot \frac{3}{4} \cdots \frac{2 N-1}{2 N}=\frac{(2 N) !}{4^{N} N !^{2}}$ and Stirling's approximation shows $\vartheta(\delta) \sim \frac{1}{\sqrt{\pi N}} \sim \sqrt{\frac{2 \delta}{\pi}}$.

Lemma 5.14. For $\alpha \in \mathcal{V}_{\left[\mathbb{C}_{1}, 0\right]}^{c m}$, we have $\sqrt{\frac{\pi}{2 \delta}} H_{\left[\mathbb{C}_{\delta}, 0\right]}^{\delta \alpha}(z) \stackrel{\delta \downarrow 0}{\longrightarrow} \tilde{h}_{[\mathbb{C}, 0]}^{d \alpha}(z):=\frac{\tilde{C}_{\alpha}}{\sqrt{z}}$ uniformly on compact subsets in $\mathbb{C} \backslash\{0\}$ for some $\tilde{C}_{\alpha} \in \mathbb{R}$, which can be computed recursively.

Proof. [CHI15, Lemma 2.14] provides the case where $\alpha=\alpha_{0}=\frac{1}{2}^{o}, o=\left(e^{2 \cdot \pi i}\right)^{2}$ $\left(\tilde{C}_{\alpha_{0}}=\frac{1}{2 \sqrt{2}}\right.$ in our normalization). The other cases easily follow from the recursive constructions given in Proposition 3.22 and Corollary 3.24. The fact that $\tilde{C}_{\alpha} \in \mathbb{R}$ is inductively apparent from the fact that for any source point $\alpha$, the function $H_{\left[\mathbb{C}_{\delta}, 0\right]}^{\delta \alpha}(z)$ vanishes if $z$ is an imaginary corner of the positive real line, or a real corner of the negative real line.

Lemma 5.15. We have that $\sqrt{\frac{\pi}{2 \delta}} G_{\left[\mathbb{C}_{\delta}, 0\right]}(z) \stackrel{\delta \downarrow 0}{\longrightarrow} \frac{1}{2 \sqrt{2}} \sqrt{z}$ and $\sqrt{\frac{\pi}{2 \delta}} \tilde{G}_{\left[\mathbb{C}_{\delta}, 0\right]}^{ \pm}(z) \stackrel{\delta \downarrow 0}{\longrightarrow}$ $\frac{i}{2 \sqrt{2}} \sqrt{z}$ uniformly on compact subsets in $\mathbb{C} \backslash\{0\}$.

Proof. By [CHI15, Lemma 2.17], we have convergence of the real part of $G_{\left[\mathbb{C}_{\delta}, 0\right]}$. The lemma follows by rotation and multiplication by $i$.

5.4.2. Convergence of the bounded domain observables We begin by proving the following convergence result, which is a simple generalization of [CHI15, Theorem 2.18]. It provides a local estimate near the monodromy point 0 which will be crucial to the proof of the general global convergence.

As we have introduced $\tilde{h}_{[\mathbb{C}, 0]}^{d \alpha}$ as the counterpart of $h_{[\mathbb{C}, 0]}^{d \alpha}$ where $C_{\alpha}$ was replaced by $\tilde{C}_{\alpha}$ in Lemma 5.14, we define $\tilde{h}_{[\Omega, 0]}^{d \alpha_{0}}$ using Definition 5.9 where $C_{\alpha}$ in $h_{[\Omega, 0]}^{d \alpha}$ is replaced by $\tilde{C}_{\alpha}$ (i.e. $\tilde{h}_{[\Omega, 0]}^{d \alpha_{0}}(z)=\sqrt{\varphi^{\prime}(z)} \frac{\tilde{C}_{\alpha}}{\sqrt{\varphi(z)}}$ ). Note $C_{\alpha_{0}}=\tilde{C}_{\alpha_{0}}=\frac{1}{2 \sqrt{2}}$. Recall also that we defined $\mathcal{A}_{\Omega}$ as the coefficient in the expansion,

$$
h_{[\Omega, 0]}^{d \alpha_{0}}(z)=\tilde{h}_{[\Omega, 0]}^{d \alpha_{0}}(z)=\frac{1}{2 \sqrt{2}}\left(\frac{1}{\sqrt{z}}+2 \mathcal{A}_{\Omega} \sqrt{z}+O\left(|z|^{3 / 2}\right)\right) .
$$

Lemma 5.16. For $\alpha_{0}=\frac{1}{2}^{o}, \sqrt{o}=1$, and a corner or medial vertex a on $\left[\mathbb{C}_{1}, 0\right]$,

$$
\begin{aligned}
H_{\left[\Omega_{\delta}, 0\right]}^{\dagger \delta \alpha_{0}}(\delta a) & =H_{\left[\Omega_{\delta}, 0\right]}^{\delta \alpha_{0}}(\delta a)-H_{\left[\mathbb{C}_{\delta}, 0\right]}^{\delta \alpha_{0}}(\delta a) \\
& =\left(2 \operatorname{Re} \mathcal{A}_{\Omega} \cdot G_{\left[\mathbb{C}_{\delta}, 0\right]}+2 \operatorname{Im} \mathcal{A}_{\Omega} \cdot \tilde{G}_{\left[\mathbb{C}_{\delta}, 0\right]}^{-}\right)(\delta a)+o(\delta) .
\end{aligned}
$$

Proof. We closely follow the strategy in Section 3.5 of [CHI15]. Note that

$$
H_{\left[\Omega_{\delta}, 0\right]}^{\dagger \delta \alpha_{0}}-\left(2 \operatorname{Re} \mathcal{A}_{\Omega} \cdot G_{\left[\mathbb{C}_{\delta}, 0\right]}+2 \operatorname{Im} \mathcal{A}_{\Omega} \cdot \tilde{G}_{\left[\mathbb{C}_{\delta}, 0\right]}^{-}\right)
$$

is s-holomorphic, so it suffices to show that it is $o(\delta)$ on real and imaginary corners and propagate. 
Recalling the symmetrized and antisymmetrized observables $S_{\left[\Omega_{\delta}, 0\right]}^{\alpha}:=\frac{1}{2}\left[H_{\left[\Omega_{\delta}, 0\right]}^{\alpha}+\right.$ $\left.H_{\overline{\left[\Omega_{\delta}, 0\right]}}^{\bar{\alpha}}\right], A_{\left[\Omega_{\delta}, 0\right]}^{\alpha}:=\frac{1}{2}\left[H_{\left[\Omega_{\delta}, 0\right]}^{\alpha}-H_{\overline{\left[\Omega_{\delta}, 0\right]}}^{\bar{\alpha}}\right]$ and $\Lambda_{\delta}=\Omega_{\delta} \cap \overline{\Omega_{\delta}}$ from Definition 3.11, define the following functions, s-holomorphic everywhere on $\left[\Lambda_{\delta}, 0\right]$ :

$$
\begin{aligned}
& S_{\delta}:=S_{\left[\Omega_{\delta}, 0\right]}^{\delta \alpha_{0}}-S_{\left[\mathbb{C}_{\delta}, 0\right]}^{\delta \alpha_{0}}-2 \operatorname{Re} \mathcal{A}_{\Omega} \cdot G_{\left[\mathbb{C}_{\delta}, 0\right]}, \\
& A_{\delta}:=A_{\left[\Omega_{\delta}, 0\right]}^{\delta \alpha_{0}}-A_{\left[\mathbb{C}_{\delta}, 0\right]}^{\delta \alpha_{0}}-2 \operatorname{Im} \mathcal{A}_{\Omega} \cdot \tilde{G}_{\left[\mathbb{C}_{\delta}, 0\right]}^{-} \cdot
\end{aligned}
$$

Given that $S_{\delta}+A_{\delta}=H_{\left[\Omega_{\delta}, 0\right]}^{\dagger \alpha_{0}}-\left(2 \operatorname{Re} \mathcal{A}_{\Omega} \cdot G_{\left[\mathbb{C}_{\delta}, 0\right]}+2 \operatorname{Im} \mathcal{A}_{\Omega} \cdot \tilde{G}_{\left[\mathbb{C}_{\delta}, 0\right]}\right)$, it remains to estimate the real and imaginary parts $S_{\delta}^{1}:=\left.S_{\delta}\right|_{\mathcal{V}_{\left[\Lambda_{\delta}, 0\right]}^{1} \cap \mathbb{X}^{+}}, S_{\delta}^{i}:=\left.S_{\delta}\right|_{\mathcal{V}_{\left[\Lambda_{\delta}, 0\right]}^{i} \cap \mathbb{Y}^{+}}$and $A_{\delta}^{1}:=\left.A_{\delta}\right|_{\mathcal{V}_{\left[\Lambda_{\delta}, 0\right]}^{1} \cap \mathbb{Y}^{+}}, A_{\delta}^{i}:=\left.A_{\delta}\right|_{\mathcal{V}_{\left[\Lambda_{\delta}, 0\right]}^{i} \cap \mathbb{X}^{+}}$. Without loss of generality we show the $o(\delta)$ estimate for $S_{\delta}^{1}(\delta a)$, where $S_{\delta}^{1}$ is harmonic in the slit domain $\mathcal{V}_{\left[\Lambda_{\delta}, 0\right]}^{1} \cap \mathbb{X}^{+}$and vanishes on $\mathcal{V}_{\left[\Lambda_{\delta}, 0\right]}^{1} \cap \mathbb{R}_{<0}$.

Define the discrete circle $w(r):=\left\{z \in \operatorname{Dom}\left(S_{\delta}^{1}\right): r<|z|<r+5 \delta\right\}$ for small $r>0$. The same twist of the discrete Beurling estimate ([LaLi04, Theorem 1]) as in $\left[\right.$ CHI15, Lemma 3.3] or our proof of Theorem 3.15 gives $\mathrm{hm}_{\{\delta a\}}^{\mathbb{X}_{\delta}^{+}}(z) \leq C \delta^{1 / 2}|z|^{-1 / 2}$. By reversibility of the simple random walk we have $\operatorname{hm}_{w(r)}^{\mathbb{X}_{\delta}^{+}}(\delta a) \leq C \delta^{1 / 2} r^{-1 / 2}$, which gives an estimate of a harmonic function identically 1 on $w(r)$ and vanishing on the slit. Comparing this with $S_{\delta}^{1}$ on $w(r)$ and applying the maximum principle in the interior gives

$$
\left|S_{\delta}^{1}(\delta a)\right| \leq C \delta^{\frac{1}{2}} r^{-\frac{1}{2}} \sup _{w(r)}\left|S_{\delta}^{1}\right|
$$

Now by convergence of $\sqrt{\frac{\pi}{2 \delta}} H_{\left[\Omega_{\delta}, 0\right]}^{\delta \alpha_{0}}$ to $h_{[\Omega, 0]}^{d \alpha_{0}}$, away from the singularity, as $\delta \rightarrow 0$ (see Theorem 2.16 of [CHI15]), we have

$$
\begin{aligned}
\sqrt{\frac{\pi}{2 \delta}} S_{\delta}^{1}(z) \rightarrow & \operatorname{Re}\left[\frac{1}{2}\left(\tilde{h}_{[\Omega, 0]}^{d \alpha_{0}}(z)+\overline{\tilde{h}_{[\Omega, 0]}^{d \alpha_{0}}(\bar{z})}\right)-\tilde{h}_{[\mathbb{C}, 0]}^{d \alpha_{0}}(z)-2 \mathcal{A}_{\Omega} \operatorname{Re} \sqrt{z}\right] \\
= & O\left(|z|^{3 / 2}\right) .
\end{aligned}
$$

Here we used the fact that $\tilde{h}_{\overline{[\Omega, 0]}}^{\overline{d \alpha_{0}}}(\cdot)=\overline{\tilde{h}_{[\Omega, 0]}^{d \alpha_{0}(\cdot)}}$ since the right hand side is the unique solution to the boundary value problem in Remark 5.10. Thus, we have $\left|S_{\delta}^{1}(\delta a)\right| \leq C^{\prime} \delta r$ and since $r$ is arbitrary we have $S_{\delta}^{1}(\delta a)=o(\delta)$ as $\delta \rightarrow 0$.

The estimate follows analogously for $S_{\delta}^{i}, A_{\delta}^{1}, A_{\delta}^{i}$, since they share the following properties which were the two properties needed to deduce that $S_{\delta}^{1}(\delta a)=o(\delta)$ above:

1. they are harmonic functions on their respective slit domains and vanish on the slits; we might extend the slit by a point, specifically the slit for $A_{\delta}^{i}$ includes $\frac{\delta}{2} \in$ $\mathcal{V}_{\left[\Lambda_{\delta}, 0\right]}^{i} \cap \mathbb{X}^{+}$given that $A_{\delta}\left(\frac{\delta}{2}\right)=A_{\delta}^{i}\left(\frac{\delta}{2}\right)=0$ (which also implies that $A_{\delta}^{1}$ is harmonic at $\left.-\frac{\delta}{2}\right)$.

2. they are $O\left(|z|^{3 / 2}\right)$ on the discrete circle $w(r)$ defined above.

Now we prove global convergence for general source point $\alpha \neq \alpha_{0}$, away from 0 . 
Proposition 5.17. If $\alpha=a^{o}$ is an s-oriented corner or medial vertex on $\left[\mathbb{C}_{1}, 0\right]$; then as $\delta \rightarrow 0$, we have $\sqrt{\frac{\pi}{2 \delta}} H_{\left[\Omega_{\delta}, 0\right]}^{\delta \alpha}(z) \stackrel{\delta \downarrow 0}{\longrightarrow} \tilde{h}_{[\Omega, 0]}^{d \alpha}(z)$ uniformly on compact subsets of $\Omega \backslash\{0\}$.

Proof. [CHI15, Theorem 2.16] proves the case where $\alpha=\alpha_{0}=\frac{1}{2}^{o}, \sqrt{o}^{o}=1$; they consider the square integrals $\tilde{Q}_{\delta}^{\delta \alpha_{0}}$ and $Q_{\delta}^{\dagger \delta \alpha_{0}}$ as introduced in Sect. 5.1 (in their notation, $\left.H_{\delta}, H_{\delta}^{\dagger}\right)$, and show that they converge to the continuous functions $\operatorname{Re} \int\left(\tilde{h}_{[\Omega, 0]}^{\delta \alpha_{0}}\right)^{2}$ and $\operatorname{Re} \int\left(\tilde{h}_{[\Omega, 0]}^{\delta \alpha_{0}}-\tilde{h}_{[\mathbb{C}, 0]}^{\delta \alpha_{0}}\right)^{2}$, which implies convergence of the integrand (see Section 3.4 of [CHI15]). In our notation, they show uniform boundedness, and thus equicontinuity, of $\tilde{Q}_{\delta}^{\delta \alpha_{0}}, Q_{\delta}^{\dagger \delta \alpha_{0}}$ in $\delta$ in each subdomain of $\Omega_{\delta}$, away from the boundary and 0 . Their subsequential limits are then identified with the continuous square integrals above.

We argue that a similar strategy works for all $\alpha$. In fact, the only difference here is that the sub-harmonicity of $Q_{\delta}^{\dagger \delta \alpha}$ at 0 fails, since in general $H_{\left[\Omega_{\delta}, 0\right]}^{\dagger \delta \alpha}\left(\frac{\delta}{2}\right) \neq 0$. Sub-harmonicity of the square integral is used twice in the proof of [CHI15, Theorem 2.16]: it shows that their $H_{\delta}$ (which corresponds to our $Q_{\delta}^{\dagger \delta \alpha}$ ) is uniformly bounded near 0 , which is needed to identify the limit, and it is needed to apply the maximum principle near 0 and obtain [CHI15, Lemma 3.10]. We will thus reproduce these two bounds, except that we replace $Q_{\delta}^{\dagger \delta \alpha}$ in their argument by a modified version $Q_{\delta}^{\dagger \dagger \delta \alpha}$, which we now introduce.

By Lemma 5.16 and rescaling,

$$
H_{\left[\Omega_{\delta}, 0\right]}^{\dagger \delta \alpha_{0}}(\delta a)=\delta\left(2 \operatorname{Re} \mathcal{A}_{\Omega} \cdot G_{\left[\mathbb{C}_{1}, 0\right]}(a)+2 \operatorname{Im} \mathcal{A}_{\Omega} \cdot \tilde{G}_{\left[\mathbb{C}_{1}, 0\right]}^{-}(a)+o(1)\right),
$$

and by antisymmetry between the two arguments, it is easy to see that $H_{\left[\Omega_{\delta}, 0\right]}^{\dagger \delta \alpha}\left(\delta a_{0}\right)=$ $\frac{1}{2 \sqrt{2}}\left(A_{\alpha}+o(1)\right) i \delta$ as $\delta \rightarrow 0$ for some constant $A_{\alpha}$. Then the modified observable

$$
H_{\left[\Omega_{\delta}, 0\right]}^{\dagger \dagger \delta \alpha}:=H_{\left[\Omega_{\delta}, 0\right]}^{\dagger \delta \alpha}-2 \sqrt{2} \frac{H_{\left[\Omega_{\delta}, 0\right]}^{\dagger \delta \alpha}\left(\delta a_{0}\right)}{\delta i} \tilde{G}_{\left[\mathbb{C}_{\delta}, 0\right]}^{+}=H_{\left[\Omega_{\delta}, 0\right]}^{\dagger \delta \alpha}-\left(A_{\alpha}+o(1)\right) \tilde{G}_{\left[\mathbb{C}_{\delta}, 0\right]}^{+}
$$

is everywhere s-holomorphic and satisfies $H_{\left[\Omega_{\delta}, 0\right]}^{\dagger \dagger \delta \alpha}\left(\delta a_{0}\right)=0$, so its integral $Q_{\delta}^{\dagger \dagger \delta \alpha}:=$ $\mathbb{I}_{\delta}\left[H_{\left[\Omega_{\delta}, 0\right]}^{\dagger \dagger \delta \alpha}\right]$ is sub-harmonic on faces and super-harmonic on vertices. It converges to

$$
\operatorname{Re} \int\left(\tilde{h}_{[\Omega, 0]}^{d \alpha}(z)-\tilde{h}_{[\mathbb{C}, 0]}^{d \alpha}(z)-\frac{i}{2 \sqrt{2}} A_{\alpha} \sqrt{z}\right)^{2} d z
$$

uniformly away from 0 , so both the discrete observable and the continuous function are single-valued and bounded near 0 . This fact, alternatively to its analogue for $Q_{\delta}^{\dagger \delta \alpha}$, also implies [CHI15, (2.8)], which identifies the singularity at 0 . The analog of [CHI15, Lemma 3.10] also easily follows by replacing $H_{\delta}^{\dagger}$ in their proof by $Q_{\delta}^{\dagger \dagger \delta \alpha}$ as defined above.

We now analyze the observable near the singularity at 0 , finally giving the proof of the main convergence theorem. Balancing the two discrete analogues $\tilde{G}_{\left[\mathbb{C}_{\delta}, 0\right]}^{ \pm}$of $\frac{i}{2 \sqrt{2}} \sqrt{z}$ to create a harmonic function which is amenable to the methods of analysis used thus far is crucial to the proof. 
Theorem 5.18 (Convergence Content of Theorem 5.12). If $z \in \mathcal{V}_{\left[\mathbb{C}_{1}, 0\right]}^{c}$ and $\alpha=a^{o}$ is an s-oriented corner on $\left[\mathbb{C}_{1}, 0\right]$, then as $\delta \rightarrow 0$,

$$
\begin{aligned}
H_{\left[\Omega_{\delta}, 0\right]}^{\dagger \delta \alpha}(\delta z)= & 2 \sqrt{2} \tilde{C}_{\alpha}\left(2 \operatorname{Re} \mathcal{A}_{\Omega} \cdot G_{\left[\mathbb{C}_{\delta}, 0\right]}+2 \operatorname{Im} \mathcal{A}_{\Omega} \cdot \tilde{G}_{\left[\mathbb{C}_{\delta}, 0\right]}^{-}\right)(\delta z) \\
& +i 2 \sqrt{2} \sqrt{o}\left(2 \operatorname{Re} \mathcal{A}_{\Omega} \cdot G_{\left[\mathbb{C}_{1}, 0\right]}+2 \operatorname{Im} \mathcal{A}_{\Omega} \cdot \tilde{G}_{\left[\mathbb{C}_{1}, 0\right]}^{-}\right)(a) \\
& {\left[\tilde{G}_{\left[\mathbb{C}_{\delta}, 0\right]}^{+}-\tilde{G}_{\left[\mathbb{C}_{\delta}, 0\right]}^{-}\right](\delta z)+o(\delta) . }
\end{aligned}
$$

Proof. We argue that the same strategy as in the proof of Lemma 5.16 works here. Indeed, after defining

$$
\begin{aligned}
S_{\delta}:= & S_{\left[\Omega_{\delta}, 0\right]}^{\delta \alpha}-S_{\left[\mathbb{C}_{\delta}, 0\right]}^{\delta \alpha}-2 \cdot 2 \sqrt{2} \tilde{C}_{\alpha} \operatorname{Re} \mathcal{A}_{\Omega} \cdot G_{\left[\mathbb{C}_{\delta}, 0\right]} \\
A_{\delta}:= & A_{\left[\Omega_{\delta}, 0\right]}^{\delta \alpha}-A_{\left[\mathbb{C}_{\delta}, 0\right]}^{\delta \alpha}-2 \cdot 2 \sqrt{2} \tilde{C}_{\alpha} \operatorname{Im} \mathcal{A}_{\Omega} \cdot \tilde{G}_{\left[\mathbb{C}_{\delta}, 0\right]}^{-} \\
& -i 2 \sqrt{2} \sqrt{o}\left(2 \operatorname{Re} \mathcal{A}_{\Omega} \cdot G_{\left[\mathbb{C}_{1}, 0\right]}+2 \operatorname{Im} \mathcal{A}_{\Omega} \cdot \tilde{G}_{\left[\mathbb{C}_{1}, 0\right]}^{-}\right)(a)\left[\tilde{G}_{\left[\mathbb{C}_{\delta}, 0\right]}^{+}-\tilde{G}_{\left[\mathbb{C}_{\delta}, 0\right]}^{-}\right],
\end{aligned}
$$

one sees that the real and imaginary parts of these two functions satisfy properties (1) and (2) at the end of the proof of Lemma 5.16, sufficient to conclude that $S_{\delta}^{1}, S_{\delta}^{i}, A_{\delta}^{1}, A_{\delta}^{i}$ evaluated at $(\delta z)$ are $o(\delta)$. The additional term in $A_{\delta}$ above is needed because we require $A_{\delta}\left(\delta a_{0}\right)=0$;

$$
\begin{aligned}
{\left[A_{\left[\Omega_{\delta}, 0\right]}^{\delta \alpha}-A_{\left[\mathbb{C}_{\delta}, 0\right]}^{\delta \alpha}\right]\left(\delta a_{0}\right) } & =H_{\left[\Omega_{\delta}, 0\right]}^{\dagger \delta \alpha}\left(\delta a_{0}\right)=-\sqrt{o} H_{\left[\Omega_{\delta}, 0\right]}^{\dagger \delta \alpha_{0}}(\delta a) \\
& =-\delta \sqrt{o}\left(2 \operatorname{Re} \mathcal{A}_{\Omega} \cdot G_{\left[\mathbb{C}_{1}, 0\right]}+2 \operatorname{Im} \mathcal{A}_{\Omega} \cdot \tilde{G}_{\left[\mathbb{C}_{1}, 0\right]}^{-}\right)(a)+o(\delta),
\end{aligned}
$$

so we insert $\tilde{G}^{+}$to cancel out this nonzero value, then adjust the coefficient in front of $\tilde{G}^{-}$to match the global limit.

We are now in position to explicitly characterize $\tilde{C}_{\alpha}$. The following is a consequence of Theorem 5.18.

Corollary 5.19. For every $\alpha \in \mathcal{V}_{\left[\mathbb{C}_{1}, 0\right]}^{c m}$, the constant $\tilde{C}_{\alpha}$ defined in Lemma 5.14 is given explicitly by

$$
\tilde{C}_{\alpha}=-\operatorname{Re}\left[i \sqrt{o}\left(\tilde{G}_{\left[\mathbb{C}_{1}, 0\right]}^{+}-\tilde{G}_{\left[\mathbb{C}_{1}, 0\right]}^{-}\right)(a)\right]=: C_{\alpha},
$$

and therefore $\tilde{h}_{[\mathbb{C}, 0]}^{d \alpha}=h_{[\mathbb{C}, 0]}^{d \alpha}$.

Proof. First we suppose $a$ is a real or imaginary corner; note that in this case the real part operator in the definition of $C_{\alpha}$ or real observables is superfluous. In Theorem 5.18, let $z=\frac{3}{2}$, say on $\mathbb{X}^{+}$, and let $\zeta=z^{p}$ with $\sqrt{p}=i$. Since $F_{\left[\Omega_{\delta}, 0\right]}^{\dagger \delta \alpha, \delta \zeta}=\operatorname{Re} i \sqrt{p} H_{\left[\Omega_{\delta}, 0\right]}^{\dagger \delta \alpha}(\delta z)=$ $i \sqrt{p} H_{\left[\Omega_{\delta}, 0\right]}^{\dagger \delta \alpha}(\delta z)$,

$$
\begin{aligned}
(2 \sqrt{2} \delta)^{-1} F_{\left[\Omega_{\delta}, 0\right]}^{\dagger \delta \alpha, \delta \zeta} \rightarrow & \tilde{C}_{\alpha}\left[i \sqrt{p}\left(2 \operatorname{Re} \mathcal{A}_{\Omega} \cdot G_{\left[\mathbb{C}_{1}, 0\right]}+2 \operatorname{Im} \mathcal{A}_{\Omega} \cdot \tilde{G}_{\left[\mathbb{C}_{1}, 0\right]}^{-}\right)(z)\right] \\
& -C_{\zeta}\left[i \sqrt{o}\left(2 \operatorname{Re} \mathcal{A}_{\Omega} \cdot G_{\left[\mathbb{C}_{1}, 0\right]}+2 \operatorname{Im} \mathcal{A}_{\Omega} \cdot \tilde{G}_{\left[\mathbb{C}_{1}, 0\right]}^{-}\right)(a)\right], \\
(2 \sqrt{2} \delta)^{-1} F_{\left[\Omega_{\delta}, 0\right]}^{\dagger \delta \zeta, \delta \alpha} \rightarrow & \tilde{C}_{\zeta}\left[i \sqrt{o}\left(2 \operatorname{Re} \mathcal{A}_{\Omega} \cdot G_{\left[\mathbb{C}_{1}, 0\right]}+2 \operatorname{Im} \mathcal{A}_{\Omega} \cdot \tilde{G}_{\left[\mathbb{C}_{1}, 0\right]}^{-}\right)(a)\right]
\end{aligned}
$$




$$
-C_{\alpha}\left[i \sqrt{p}\left(2 \operatorname{Re} \mathcal{A}_{\Omega} \cdot G_{\left[\mathbb{C}_{1}, 0\right]}+2 \operatorname{Im} \mathcal{A}_{\Omega} \cdot \tilde{G}_{\left[\mathbb{C}_{1}, 0\right]}^{-}\right)(z)\right] .
$$

Since the two limits should differ only by sign, the result follows by using that $G_{\left[\mathbb{C}_{1}, 0\right]}(z) \neq 0, \tilde{G}_{\left[\mathbb{C}_{1}, 0\right]}^{ \pm}(z)=C_{\zeta}=0$, and the fact that, by our recursive construction, $\tilde{C}_{\zeta}=0$ (see Proposition 3.22, and in particular, Proposition A.2).

In the case where $a$ is a medial vertex, note that by s-holomorphicity of $H_{\left[\mathbb{C}_{\delta}, 0\right]}^{\delta \alpha}$ and antisymmetry of its real counterpart, in their arguments, we can express $\tilde{C}_{\alpha}$ as a linear combination of $\tilde{C}_{\beta^{ \pm}}$, where $\beta^{ \pm}$are adjacent real and imaginary (s-oriented) corners. Notice that this linear combination is exactly mirrored in the case of $C_{\alpha}$, since $\tilde{G}_{\left[\mathbb{C}_{1}, 0\right]}^{+}-\tilde{G}_{\left[\mathbb{C}_{1}, 0\right]}^{-}$is s-holomorphic; thus the desired equality holds.

\section{Proofs of Theorems}

In this section, we complete the proofs of Theorems 1.1-1.2 and Corollary 1.3.

6.1. Spin-symmetric fields. We first prove Theorem (1.1), establishing the conformal invariance of spin-symmetric fields.

Definition 6.1. Suppose $a \neq z$ are medial vertices on $\mathbb{C}_{1}$. For s-orientations $o, p$ on $a, z$ respectively, write $\alpha=a^{o}, \zeta=z^{p}$. Define

$$
\begin{aligned}
& F_{\mathbb{C}_{1}}^{\alpha, \zeta}=\operatorname{Re}\left[i \sqrt{p} H_{\mathbb{C}_{1}}^{\alpha}(z)\right], \\
& E_{\mathbb{C}_{1}}^{\alpha, \zeta}=\operatorname{Re}\left[\frac{i \sqrt{p} \overline{\sqrt{o}}}{\sqrt{2} \pi}\right],
\end{aligned}
$$

where $H_{\mathbb{C}_{1}}^{\alpha}(z)$ was explicitly defined in (3.1).

Now let $\left\{e_{k}\right\}=\left\{e_{1}, \ldots, e_{n}\right\}$ be a collection of distinct edges of $\mathbb{C}_{1}$. Set

$$
\left(x_{1}, \ldots, x_{n}, x_{n+1}, \ldots, x_{2 n}\right):=\left(e_{1}^{+}, \ldots e_{n}^{+}, e_{n}^{-}, \ldots, e_{1}^{-}\right),
$$

where $e_{j}^{+}:=e_{j}^{o^{+}}$and $e_{j}^{-}:=e_{j}^{o^{-}}$denote a choice of opposite s-orientations of $e_{j}$ such that $o^{+}=e^{\pi i} o^{-}$.

We write $\mathbf{F}^{\left\{e_{k}\right\}}$ to denote the $2 n \times 2 n$ antisymmetric matrix with entries $\left(\mathbf{F}^{\left\{e_{k}\right\}}\right)_{j k}:=$ $F_{\mathbb{C}_{1}}^{x_{j}, x_{k}}$ for $j+k \neq 2 n+1$, and $\left(\mathbf{F}^{\left\{e_{k}\right\}}\right)_{j k}:=0$ on the anti-diagonal $j+k=2 n+1$; we also set $\mathbf{E}^{\left\{e_{k}\right\}}$ to be the matrix taking values $\left(\mathbf{E}^{\left\{e_{k}\right\}}\right)_{j k}:=E_{\mathbb{C}_{1}}^{x_{j}, x_{k}}$. Now define

$$
\begin{aligned}
& \mathcal{P}^{\left\{e_{k}\right\}}:=(-2)^{n} \operatorname{Pf}\left(\mathbf{F}^{\left\{e_{k}\right\}}\right), \\
& \mathcal{Q}^{\left\{e_{k}\right\}}:=(-2)^{n} D_{\mathbf{E}^{\left\{e_{k}\right\}}} \operatorname{Pf}\left(\mathbf{F}^{\left\{e_{k}\right\}}\right),
\end{aligned}
$$

where $D_{\mathbf{E}^{\left\{e_{k}\right\}}} \mathrm{Pf}$ denotes the directional derivative of the Pfaffian function in the direction of $\mathbf{E}^{\left\{e_{k}\right\}}$.

Remark 6.2. The values $\mathcal{P}^{\left\{e_{k}\right\}}$ and $\mathcal{Q}^{\left\{e_{k}\right\}}$ only depend on the unordered collection $\left\{e_{k}\right\}$ since they arise as limits of the Pfaffian from Proposition 4.16, which has an interpretation as a physical quantity only depending on $\left\{e_{k}\right\}$. 
Theorem 6.3 (Restatement of Theorem 1.1). Let $\left\{e_{k}\right\}_{k=1}^{n}$ be a collection of $n$ distinct edges of $\mathbb{C}_{1}$. As $\delta \rightarrow 0$, we have

$$
\mathbb{E}_{\Omega_{\delta}}\left[\prod_{e \in\left\{e_{k}\right\}} \epsilon(\delta e)\right]=\mathcal{P}^{\left\{e_{k}\right\}}+\delta \cdot r_{\Omega}^{-1}(0) \cdot \mathcal{Q}^{\left\{e_{k}\right\}}+o(\delta),
$$

where $r_{\Omega}(z)$ is the conformal radius of $\Omega$ at $z \in \Omega$, defined $r_{\Omega}(z):=\left|\varphi^{\prime}(0)\right|$ where $\varphi: \mathbb{D} \rightarrow \Omega$ is a conformal map with $\varphi(0)=z$.

Proof of Theorem 6.3. By Proposition 4.16, we have that

$$
\mathbb{E}_{\Omega_{\delta}}\left[\prod_{e \in\left\{e_{k}\right\}} \epsilon(\delta e)\right]=(-1)^{n} 2^{n} \operatorname{Pf}\left(\mathbf{F}_{\Omega_{\delta}}^{\left[\left\{\delta e_{k}\right\}\right]}\right) .
$$

Write $\mathbf{F}_{\Omega_{\delta}}^{\left[\left\{\delta e_{k}\right\}\right]}=\mathbf{F}_{\mathbb{C}_{\delta}}^{\left[\left\{\delta e_{k}\right\}\right]}+\left[\mathbf{F}_{\Omega_{\delta}}^{\left[\left\{\delta e_{k}\right\}\right]}-\mathbf{F}_{\mathbb{C}_{\delta}}^{\left.\left[\left\{\delta e_{k}\right\}\right]\right]}\right.$. By scale invariance, $\mathbf{F}_{\mathbb{C}_{\delta}}^{\left[\left\{\delta e_{k}\right\}\right]}=\mathbf{F}_{\mathbb{C}_{1}}^{\left[\left\{e_{k}\right\}\right]}$, which, by definition, satisfies $\mathbf{F}_{\mathbb{C}_{1}}^{\left[\left\{e_{k}\right\}\right]}=\mathbf{F}^{\left\{e_{k}\right\}}$.

By Theorem 5.11, for any $\alpha=a^{o}, \zeta=z^{p}$, if we set $\delta \alpha:=(\delta a)^{o}, \delta \zeta:=(\delta z)^{p}$ we can calculate

$$
\lim _{\delta \rightarrow 0} \frac{1}{\delta}\left[F_{\Omega_{\delta}}^{\delta \alpha, \delta \zeta}-F_{\mathbb{C}_{\delta}}^{\delta \alpha, \delta \zeta}\right]=\left[f_{\Omega}^{0^{o}}-f_{\mathbb{C}}^{0^{o}}\right]\left(0^{p}\right)=E_{\mathbb{C}_{1}}^{\alpha, \zeta} r_{\Omega}^{-1}(0)
$$

and the result follows from Taylor expansion of $\operatorname{Pf}\left(\mathbf{F}_{\Omega_{\delta}}^{\left[\left\{\delta e_{k}\right\}\right]}\right)=\operatorname{Pf}\left(\mathbf{F}^{\left\{e_{k}\right\}}+\delta \mathbf{E}^{\left\{e_{k}\right\}} r_{\Omega}^{-1}(0)\right.$ $+o(\delta))$.

6.2. Spin-antisymmetric fields. We now generalize the above proof to spin-antisymmetric fields, proving Theorem 1.2.

For any s-oriented medial vertex or corner $\zeta=z^{p}$, we introduce the real quantity

$$
G_{\left[\mathbb{C}_{1}, 0\right]}(\zeta)=\operatorname{Re}\left[i \sqrt{p} G_{\left[\mathbb{C}_{1}, 0\right]}(z)\right]
$$

and define the real quantities $\tilde{G}_{\left[\mathbb{C}_{1}, 0\right]}^{ \pm}(\zeta)$ analogously; $G_{\left[\mathbb{C}_{1}, 0\right]}(z), \tilde{G}_{\left[\mathbb{C}_{1}, 0\right]}^{ \pm}(z)$ were defined in Sect. 3.2.

Definition 6.4. Suppose $a \neq z$ are medial vertices on $\left[\mathbb{C}_{1}, 0\right]$. For s-orientations $o, p$ respectively on $a, z$, write $\alpha=a^{o}, \zeta=z^{p}$ for the s-oriented medial vertices. Set

$$
\begin{aligned}
& F_{\left[\mathbb{C}_{1}, 0\right]}^{\alpha, \zeta}=\operatorname{Re} {\left[i \sqrt{p} H_{\left[\mathbb{C}_{1}, 0\right]}^{\alpha}(z)\right], } \\
& E_{\left[\mathbb{C}_{1}, 0\right]}^{\alpha, \zeta}:=2 \cdot 2 \sqrt{2}\left(\left[G_{\left[\mathbb{C}_{1}, 0\right]}-i \tilde{G}_{\left[\mathbb{C}_{1}, 0\right]}^{-}\right](\alpha)\left[\tilde{G}_{\left[\mathbb{C}_{1}, 0\right]}^{+}-\tilde{G}_{\left[\mathbb{C}_{1}, 0\right]}^{-}\right](\zeta)\right. \\
& \\
&\left.\quad-\left[G_{\left[\mathbb{C}_{1}, 0\right]}-i \tilde{G}_{\left[\mathbb{C}_{1}, 0\right]}^{-}\right](\zeta)\left[\tilde{G}_{\left[\mathbb{C}_{1}, 0\right]}^{+}-\tilde{G}_{\left[\mathbb{C}_{1}, 0\right]}^{-}\right](\alpha)\right) .
\end{aligned}
$$

Let $\left\{e_{k}\right\}=\left\{e_{1}, \ldots, e_{n}\right\}$ be a collection of distinct edges of $\mathbb{C}_{1}$. Let $\tilde{e}_{1}, \ldots, \tilde{e}_{n}$ be a choice of lifts of $e_{1}, \ldots, e_{n}$ to $\left[\mathbb{C}_{1}, 0\right]$. Set

$$
\left(x_{1}, \ldots, x_{2 n}\right):=\left(\tilde{e}_{1}^{+}, \ldots, \tilde{e}_{n}^{+}, \tilde{e}_{n}^{-}, \ldots, \tilde{e}_{1}^{-}\right),
$$


where $\tilde{e}_{j}^{+}:=\tilde{e}_{j}^{o^{+}}$and $\tilde{e}_{j}^{-}:=\tilde{e}_{j}^{o^{-}}$denote a choice of opposite s-orientations of $\tilde{e}_{j}$ such that $o^{+}=e^{\pi i} o^{-}$.

Define $\mathbf{F}_{[0]}^{\left\{e_{k}\right\}}$ as the $2 n \times 2 n$ antisymmetric matrix with entries $\left(\mathbf{F}_{[0]}^{\left\{e_{k}\right\}}\right)_{j k}:=F_{\left[\mathbb{C}_{\delta}, 0\right]}^{x_{j} x_{k}}$ for $j+k \neq 2 n+1$, and $\left(\mathbf{F}_{[0]}^{\left\{e_{k}\right\}}\right)_{j k}:=0$ on the anti-diagonal, $j+k=2 n+1$. Define also $\mathbf{E}_{[0]}^{\left\{e_{k}\right\}}$ the $2 n \times 2 n$ antisymmetric matrix given by $\left(\mathbf{E}_{[0]}^{\left\{e_{k}\right\}}\right)_{j k}:=E_{\left[\mathbb{C}_{\delta}, 0\right]}^{x_{j} x_{k}}$. Let

$$
\begin{aligned}
& \mathcal{P}_{[0]}^{\left\{e_{k}\right\}}:=(-2)^{n} \operatorname{Pf}\left(\mathbf{F}_{[0]}^{\left\{e_{k}\right\}}\right), \\
& \mathcal{Q}_{[0]}^{\left\{e_{k}\right\}}:=(-2)^{n} D_{\mathbf{E}_{[0]}^{\left\{e_{k}\right\}}} \operatorname{Pf}\left(\mathbf{F}_{[0]}^{\left\{e_{k}\right\}}\right) .
\end{aligned}
$$

Remark 6.5. By the same reasoning as in the spin-symmetric case, the values $\mathcal{P}^{\left\{e_{k}\right\}}$ and $\mathcal{Q}^{\left\{e_{k}\right\}}$ only depend on the unordered collection $\left\{e_{k}\right\}$.

Theorem 6.6 (Restatement of Theorem 1.2). Let $\left\{e_{k}\right\}_{k=1}^{n}$ be a set of $n$ edges of $\mathbb{C}_{1}$. For every $1 \leq k \leq n$, the quantity $\mu_{e_{k}}$ defined in (1.1) exists and, independently of s-orientation $o_{k}$ on $e_{k}$, is

$$
\mu_{e_{k}}=\sqrt{o_{k}}\left[H_{\left[\mathbb{C}_{1}, 0\right]}^{e_{k}^{o_{k}}}\left(e_{k+}^{o_{k}}\right)+H_{\left[\mathbb{C}_{1}, 0\right]}^{e_{k}^{o_{k}}}\left(e_{k-}^{o_{k}}\right)\right]
$$

so that $\epsilon_{[0]}\left(\delta e_{k}\right)$ is a well-defined random variable for every $k$, and as $\delta \rightarrow 0$,

$$
\frac{\mathbb{E}_{\Omega_{\delta}}\left[\sigma_{0} \prod_{e \in\left\{e_{k}\right\}} \epsilon_{[0]}(\delta e)\right]}{\mathbb{E}_{\Omega_{\delta}}\left[\sigma_{0}\right]}=\mathcal{P}_{[0]}^{\left\{e_{k}\right\}}+\delta \cdot \operatorname{Re}\left[-\left.\frac{1}{4} \partial_{z} \log r_{\Omega}(z)\right|_{z=0} \cdot \mathcal{Q}_{[0]}^{\left\{e_{k}\right\}}\right]+o(\delta),
$$

where $\partial_{z}=\frac{1}{2}\left(\partial_{x}-i \partial_{y}\right)$ if $z=x+i y$. In particular, it follows from the results of [CHII5] that

$$
\mathbb{E}_{\Omega_{\delta}}\left[\sigma_{0} \prod_{a \in\left\{e_{k}\right\}} \epsilon_{[0]}(\delta e)\right]=0+\mathcal{C} \cdot \delta^{\frac{1}{8}} \cdot \mathcal{P}_{[0]}^{\left\{e_{k}\right\}} \cdot 2^{\frac{1}{4}} \cdot r_{\Omega}(0)^{-\frac{1}{8}}+o\left(\delta^{\frac{1}{8}}\right),
$$

where $\mathcal{C}$ is a constant given explicitly by Eq. (1.1) of [CHI15].

Proof of Theorem 6.6. The expression for $\mu_{e}$ for every $e \in \mathcal{E}_{\mathbb{C}_{1}}$ was given by Remark 4.8. Now by Proposition 4.17, we have that

$$
\frac{\mathbb{E}_{\Omega_{\delta}\left[\sigma_{0} \prod_{e \in\left\{e_{k}\right\}} \epsilon_{[0]}(\delta e)\right]}}{\mathbb{E}_{\Omega_{\delta}}\left[\sigma_{0}\right]}=(-1)^{n} 2^{n} \operatorname{Pf}\left(\mathbf{F}_{\left[\Omega_{\delta}, 0\right]}^{\left\{\delta e_{k}\right\}}\right) .
$$

Write $\mathbf{F}_{\left[\Omega_{\delta}, 0\right]}^{\left\{\delta e_{k}\right\}}=\mathbf{F}_{\left[\mathbb{C}_{\delta}, 0\right]}^{\left\{\delta e_{k}\right\}}+\left[\mathbf{F}_{\left[\Omega_{\delta}, 0\right]}^{\left\{\delta e_{k}\right\}}-\mathbf{F}_{\left[\mathbb{C}_{\delta}, 0\right]}^{\left\{\delta e_{k}\right\}}\right]$

As before, by scale invariance, $\mathbf{F}_{\left[\mathbb{C}_{\delta}, 0\right]}^{\left\{\delta e_{k}\right\}}=\mathbf{F}_{\left[\mathbb{C}_{1}, 0\right]}^{\left\{e_{k}\right\}}$, and by definition, $\mathbf{F}_{\left[\mathbb{C}_{1}, 0\right]}^{\left\{e_{k}\right\}}=\mathbf{F}_{[0]}^{\left\{e_{k}\right\}}$. By Theorem 5.12, for any $\alpha=a^{o}, \zeta=z^{p}$, if we set $\delta \alpha:=(\delta a)^{o}, \delta \zeta:=(\delta z)^{p}$ we have

$$
\lim _{\delta \rightarrow 0} \frac{1}{\delta} F_{\left[\Omega_{\delta}, 0\right]}^{\dagger \delta \alpha, \delta \zeta}=-\operatorname{Re}\left[\left.\frac{1}{4} \partial_{z} \log r_{\Omega}(z)\right|_{z=0} \cdot E_{[0]}^{\alpha, \zeta}\right]
$$


and hence we get

$$
\lim _{\delta \rightarrow 0} \frac{1}{\delta}\left[\mathbf{F}_{\left[\Omega_{\delta}, 0\right]}^{\left\{\delta a_{k}\right\}}-\mathbf{F}_{\left[\mathbb{C}_{\delta}, 0\right]}^{\left\{\delta a_{k}\right\}}\right]=-\operatorname{Re}\left[\left.\frac{1}{4} \partial_{z} \log r_{\Omega}(z)\right|_{z=0} \cdot \mathbf{E}_{[0]}^{\left\{a_{k}\right\}}\right] .
$$

The first result then follows from Taylor expansion as in the proof of the previous theorem, and the second follows by multiplying through by the conformally covariant expansion of $\mathbb{E}_{\Omega_{\delta}}\left[\sigma_{0}\right]=\mathcal{C} \cdot 2^{\frac{1}{4}} \cdot r_{\Omega}(0)^{-\frac{1}{8}} \cdot \delta^{\frac{1}{8}}+o\left(\delta^{\frac{1}{8}}\right)$ given by [CHI15].

6.3. Spin pattern probabilities. Finally, we prove Corollary 1.3 as a consequence of the above two proofs.

Proof of Corollary 1.3. We begin by proving the corollary for the spin-symmetric pattern fields. For a subset $\mathcal{F} \subset \mathcal{F}_{\mathbb{C}_{1}}$, let $\mathcal{B}$ be the set of all edges separating two adjacent faces in $\mathcal{F}$. To any spin-symmetric pattern $\pm \rho$ on $\mathcal{F}$, we can associate an edge subset $B \subset \mathcal{B}$ via the usual low-temperature expansion (see Fig. 1 and Sect. 3.0.1). Denote the collection of such edge subsets in $\mathcal{B}$ that are associated to a spin-symmetric pattern on $\mathcal{F}$ by $P_{\mathcal{F}}(\mathcal{B}) \subset P(\mathcal{B})$. We will index the $2^{|\mathcal{F}|-1}$-dimensional vector $P^{\mathcal{F}}=\left(P_{B}\right)_{B \in P_{\mathcal{F}}(\mathcal{B})}$ of the probabilities of all spin-symmetric patterns by corresponding edge subsets.

Given any such an edge subset $B$, we can calculate a spin-symmetric correlation $E_{B}=$ $\mathbb{E}_{\Omega_{\delta}}[\epsilon(\delta B)]=\mathbb{E}_{\Omega_{\delta}}\left[\prod_{e \in B} \epsilon(\delta e)\right]$ and also form another vector $E^{\mathcal{F}}=\left(E_{B}\right)_{B \in P_{\mathcal{F}}(\mathcal{B})}$ of dimension $2^{|\mathcal{F}|-1}$.

Clearly, every correlation function $\mathbb{E}_{\Omega_{\delta}}[\epsilon(B)]$ can be expressed as a linear combination of probabilities of the $2^{|\mathcal{F}|-1}$ spin-symmetric patterns on $\mathcal{B}$.

Thus we have a $2^{|\mathcal{F}|-1} \times 2^{|\mathcal{F}|-1}$ matrix $\left(E P^{\mathcal{F}}\right)_{B B^{\prime}}=\prod_{e \in B}\left(\mu-\left(21_{\left\{e \in B^{\prime}\right\}}-1\right)\right)$, such that $E^{\mathcal{F}}=\left(E P^{\mathcal{F}}\right) P^{\mathcal{F}}$. One can check by hand that the matrix has inverse given by:

$$
\left(P E^{\mathcal{F}}\right)_{B^{\prime} B}=\frac{1}{2^{\left(2^{|\mathcal{F}|-1}\right)}}(-1)^{\sum_{e \in \mathcal{B}} \mathbf{1}_{\{e \in B\}} \oplus \mathbf{1}_{\left\{e \in B^{\prime}\right\}}} \prod_{e \in B}\left(\mu+\left(2 \mathbf{1}_{\left\{e \in B^{\prime}\right\}}-1\right)\right) .
$$

Applying the inverse to $E^{\mathcal{F}}$, consisting of conformally covariant spin-symmetric correlations from Theorem 1.1, yields the desired result for spin-symmetric patterns.

For the spin-antisymmetric patterns, an analogous approach but conditioning on $\sigma_{0}= \pm 1$ and replacing $\mu$ by $\mu_{e}$, combined with the conformally covariant expansion $\mathbb{E}_{\Omega_{\delta}}\left[\sigma_{0}\right]=\mathcal{C} \cdot 2^{\frac{1}{4}} \cdot r_{\Omega}(0)^{-\frac{1}{8}} \cdot \delta^{\frac{1}{8}}+o\left(\delta^{1 / 8}\right)$ from [CHI15], gives the desired result.

Acknowledgements. Firstly, the authors thank the anonymous referees for their many helpful suggestions and comments. This research was initiated during the Research Experience for Undergraduates program at Mathematics Department of Columbia University, funded by the NSF under grant DMS-0739392. We would like to thank the T.A., Krzysztof Putyra, for his help during this program and the program coordinator Robert Lipshitz, as well as all the participants, in particular Adrien Brochard and Woo Chang Chung. R.G. would like to thank Chuck Newman and Eyal Lubetzky for interesting discussions. C.H. would like to thank Dmitry Chelkak and Stanislav Smirnov, for sharing many ideas and insights about the Ising model and conformal invariance; Itai Benjamini and Curtis McMullen for asking questions that suggested we look at this problem; Stéphane Benoist, John Cardy, Julien Dubédat, Hugo Duminil-Copin, Konstantin Izyurov, Kalle Kytölä and Wendelin Werner for interesting discussions, the NSF under grant DMS-1106588, the ERC under grant SG CONSTAMIS, the NCCR Swissmap, the Blavatnik family foundation, the Latsis family foundation, and the Minerva Foundation for financial support. S.P. would like to thank C. M. Munteanu for interesting discussions. 
Open Access This article is distributed under the terms of the Creative Commons Attribution 4.0 International License (http://creativecommons.org/licenses/by/4.0/), which permits unrestricted use, distribution, and reproduction in any medium, provided you give appropriate credit to the original author(s) and the source, provide a link to the Creative Commons license, and indicate if changes were made.

Publisher's Note Springer Nature remains neutral with regard to jurisdictional claims in published maps and institutional affiliations.

\section{A. The Harmonic Measure on $\mathbb{C}_{1} \backslash \mathbb{R}_{>0}$}

We start this section by giving an analytic formula for the harmonic measure of the tip of the slit plane. This uses Fourier series techniques, which was inspired by [ChHo18]. Using the formula, we prove that the auxiliary functions $G_{\left[\mathbb{C}_{1}, 0\right]}, \tilde{G}_{\left[\mathbb{C}_{1}, 0\right]}^{ \pm}$are discrete holomorphic.

Since the slit-plane harmonic measures appear as different translations of the same function, we present the following prototype:

Proposition A.1. For $s+i k \in(1+i) \mathbb{Z}^{2}$, the function $H_{0}$ given by,

$$
H_{0}(z):=h m_{0}^{(1+i) \mathbb{Z}^{2} \backslash \mathbb{Z}_{\geq 0}}(z=s+i k)=\frac{1}{2 \pi} \int_{-\pi}^{\pi} \frac{C^{|k|}(\theta)}{\sqrt{1-e^{-2 i \theta}}} e^{-i s \theta} d \theta,
$$

where $C(\theta):=\frac{\cos \theta}{1+|\sin \theta|}$ and the square root is evaluated on the principal branch, is the unique discrete harmonic function on the diagonal slit plane $(1+i) \mathbb{Z}^{2} \backslash \mathbb{Z}_{\geq 0}$ with boundary values 1 at the origin and 0 elsewhere on $\mathbb{Z}_{\geq 0}$ and as $\rightarrow \infty$.

Proof. We first state two Fourier expansions, thanks to the generalized binomial theorem:

$$
\begin{aligned}
& \frac{1}{\sqrt{1-e^{-2 i \theta}}}=\sum_{n=0}^{\infty}(-1)^{n}\left(\begin{array}{c}
-\frac{1}{2} \\
n
\end{array}\right) e^{-2 n i \theta}=1+\frac{1}{2} e^{-2 i \theta}+\frac{3}{8} e^{-4 i \theta}+\frac{5}{16} e^{-6 i \theta}+\cdots, \\
& \frac{|\sin \theta|}{\sqrt{1-e^{-2 i \theta}}}=\sqrt{\frac{\sin ^{2} \theta}{1-e^{-2 i \theta}}}=\frac{1}{2} \sqrt{1-e^{2 i \theta}}=\frac{1}{2} \sum_{n=0}^{\infty}(-1)^{n}\left(\begin{array}{c}
\frac{1}{2} \\
n
\end{array}\right) e^{2 n i \theta} .
\end{aligned}
$$

The first identity immediately gives the boundary values on $\mathbb{Z}_{\geq 0}$. Discrete harmonicity when $k \neq 0$ follows directly from the structure of the integrand, and when $k=0$ values of the discrete Laplacian correspond to the Fourier coefficients in the second identity of Eq. A.2, so vanishes on $\mathbb{Z}_{<0}$ since there are no negative Fourier modes.

For the decay at infinity, it suffices to show $\left|H_{0}(s+i k)\right| \rightarrow 0$ as $|k| \rightarrow \infty$ uniformly in $s$ and as $|s| \rightarrow \infty$ for fixed $k$. Note the latter is just the Riemann-Lebesgue Lemma. For the former, we can use dominated convergence since $\frac{\left|C^{|k|}(\theta)\right|}{\sqrt{\left|1-e^{-2 i \theta}\right|}} \downarrow 0$ pointwise a.e. as $|k| \rightarrow \infty$ and $\frac{\left|C^{|k|}(\theta)\right|}{\sqrt{\left|1-e^{-2 i \theta}\right|}} \leq \frac{1}{\sqrt{\left|1-e^{-2 i \theta}\right|}}=\frac{1}{\sqrt{2|\sin \theta|}}$, which is integrable.

Now, the above characterization of the harmonic measure of the tip of the slit plane leads to a recursive construction of harmonic measures of other points on the slit, as discussed 
in Proposition 3.22. Suppose $H_{n}:=\mathrm{hm}_{2 n}^{(1+i) \mathbb{Z}^{2}}$ denotes the harmonic measure of the point $2 n$ on the slit plane $(1+i) \mathbb{Z}^{2} \backslash \mathbb{R}_{\geq 0}$. We have the following recursion formula:

$$
\begin{aligned}
H_{n}(m) & =H_{n-1}(m-2)-H_{n-1}(-2) H_{0}(m) \\
& =: H_{0}(m-2 n)-X_{1} H_{0}(m-2 n+2)-X_{2} H_{0}(m-2 n+4)-\cdots-X_{n} H_{0}(m) .
\end{aligned}
$$

The coefficients $X_{n}:=H_{n-1}(-2)$ can be used to calculate the recursive coefficients in Proposition 3.22 and the scaling limit coefficients $C_{\alpha}$ in Sect. 5.2.1 explicitly. We now give a simple formula for $X_{i}$.

Proposition A.2. For all $i \geq 1$, we have that $X_{n}=\frac{H_{0}(-2 n+2)}{2 n}$. Consequently,

$$
1-X_{1}-X_{2}-\cdots-X_{n}=H_{0}(-2 n) \text {. }
$$

Proof. Define the generating functions

$$
\begin{aligned}
& X(z):=\sum_{n=1}^{\infty} X_{n} z^{n}=\frac{1}{2} z+\frac{1}{8} z^{2}+\frac{1}{16} z^{3}+\cdots \\
& F(z):=\sum_{n=0}^{\infty} H_{0}(-2 i) z^{n}=1+\frac{1}{2} z+\frac{3}{8} z^{2}+\cdots=\frac{1}{\sqrt{1-z}} .
\end{aligned}
$$

Note that Eq. (A.3) implies a convolution identity by taking $m=0$,

$$
H_{0}(-2 m)=\sum_{n=0}^{m} X_{n} H_{0}(-2 m+2 n)
$$

and setting $X_{0}=0$. Thus $X F=F-1$, and $X=1-\sqrt{1-z}=\sum_{n=1}^{\infty}(-1)^{n+1}\left(\begin{array}{c}1 / 2 \\ n\end{array}\right) z^{n}$. Given that $H_{0}(-2 n)=(-1)^{n}\left(\begin{array}{c}-1 / 2 \\ n\end{array}\right)$ by Eq. (A.2), both results are straightforward.

Corollary A.3. The auxiliary functions, $G_{\left[\mathbb{C}_{1}, 0\right]}, \tilde{G}_{\left[\mathbb{C}_{1}, 0\right]}^{ \pm}$, defined in Definition 3.25 are discrete holomorphic on $\left[\mathbb{C}_{1}, 0\right]$.

Proof. Without loss of generality, we will show discrete holomorphicity of $G_{\left[\mathbb{C}_{1}, 0\right]}$ at a type- $\lambda$ corner $z=s+i\left(k+\frac{1}{2}\right) \in \mathbb{X}^{+} \cap \mathbb{Y}^{+}(k \geq 0)$. By the fact that

$$
\sum_{n=-\infty}^{\infty}\left[\operatorname{hm}_{3 / 2}^{\mathbb{X}_{1}^{1}}\left(z+\frac{1+i}{2}+2 n\right)-\operatorname{hm}_{3 / 2}^{\mathbb{X}_{1}^{1}}\left(z-\frac{1+i}{2}+2 n\right)\right]=\lim _{\theta \rightarrow 0} C^{k}(\theta) \frac{C(\theta)-1}{\sqrt{1-e^{-2 i \theta}}}=0,
$$

and discrete holomorphicity of $H_{\left[\mathbb{C}_{1}, 0\right]}^{\alpha_{0}}$, we have

$$
\begin{aligned}
G_{\left[\mathbb{C}_{1}, 0\right]}\left(z-\frac{1-i}{2}\right)-G_{\left[\mathbb{C}_{1}, 0\right]}\left(z+\frac{1-i}{2}\right) \\
=\frac{i}{2 \sqrt{2}} \sum_{n=1}^{\infty}\left[\operatorname{hm}_{1 / 2}^{\mathbb{Y}_{1}^{i}}\left(z-\frac{1-i}{2}+2 n\right)-\mathrm{hm}_{1 / 2}^{\left.\mathbb{Y}_{1}^{i}\left(z+\frac{1-i}{2}+2 n\right)\right]}\right. \\
=-\frac{i}{2 \sqrt{2}} \sum_{n=1}^{\infty}\left[\operatorname{hm}_{3 / 2}^{\mathbb{X}_{1}^{1}}\left(z+\frac{1+i}{2}+2 n\right)-\mathrm{hm}_{3 / 2}^{\mathbb{X}_{1}^{1}}\left(z-\frac{1+i}{2}+2 n\right)\right]
\end{aligned}
$$




$$
\begin{aligned}
& =\frac{i}{2 \sqrt{2}} \sum_{n=0}^{\infty}\left[\operatorname{hm}_{3 / 2}^{\mathbb{X}_{1}^{1}}\left(z+\frac{1+i}{2}-2 n\right)-\mathrm{hm}_{3 / 2}^{\mathbb{X}_{1}^{1}}\left(z-\frac{1+i}{2}-2 n\right)\right] \\
& =i\left[G_{\left[\mathbb{C}_{1}, 0\right]}\left(z+\frac{1+i}{2}\right)-G_{\left[\mathbb{C}_{1}, 0\right]}\left(z-\frac{1+i}{2}\right)\right],
\end{aligned}
$$

as desired.

Now we show that $G$ in fact has some rotation symmetry, which can be exploited to recursively compute its values as outlined in Remark 3.27. The proof relies on the same kind of analysis of discrete harmonic functions as in the proof of Lemma 5.16 and therefore we omit some of the details.

Proposition A.4. On $\mathbb{C}_{\delta}$, the following holds: $e^{\pi i / 4} \cdot G_{\left[\mathbb{C}_{\delta}, 0\right]}\left(e^{\pi i / 2} z\right)=$ $\frac{1}{2}\left[\tilde{G}_{\left[\mathbb{C}_{\delta}, 0\right]}^{+}+\tilde{G}_{\left[\mathbb{C}_{\delta}, 0\right]}^{-}\right](z)$.

Proof. We will write $L_{\delta}(z)$ for the left hand side, and $R_{\delta}(z)=\left[k \tilde{G}_{\left[\mathbb{C}_{\delta}, 0\right]}^{+}+\right.$ $\left.(1-k) \tilde{G}_{\left[\mathbb{C}_{\delta}, 0\right]}^{-}\right](z)$ for an as yet undefined real number $k$. Both are s-holomorphic functions, and by Lemma 5.15, both $\sqrt{\frac{\pi}{2 \delta}} L_{\delta}(z), \sqrt{\frac{\pi}{2 \delta}} R_{\delta}(z)$ converge to $\frac{i}{2 \sqrt{2}} \sqrt{z}$ on compact subsets away from 0 as $\delta \rightarrow 0$.

First, it is straightforward to check that $G_{\left[\mathbb{C}_{\delta}, 0\right]}$ vanishes on $\bar{\lambda}$-corners on the upper half of the imaginary axis, and on $\lambda$-corners on the lower half by the symmetry between its real and imaginary parts (in addition, $\left.G_{\left[\mathbb{C}_{\delta}, 0\right]}(\bar{z})=\overline{G_{\left[\mathbb{C}_{\delta}, 0\right]}(z)}\right)$. So $L_{\delta}(z)$ has zero real part on the positive real line, and zero imaginary part on the negative real line. This is also true for $R_{\delta}(z)$. Now, $L_{\delta}\left( \pm \frac{\delta}{2}\right)$ is not necessarily zero, so we will choose (taking $\frac{\delta}{2}$ on $\left.\mathbb{X}^{+}\right) k=-i L_{\delta}\left(\frac{\delta}{2}\right)$. Then $L_{\delta}(z)-R_{\delta}(z)$ is zero at $\frac{\delta}{2}$, so we have harmonicity at $-\frac{\delta}{2}$ by Remark 2.6.

Without loss of generality, consider the restriction of $\sqrt{\frac{\pi}{2 \delta}}\left[L_{\delta}-R_{\delta}\right]$ to $\mathcal{V}_{\left[\mathbb{C}_{\delta}, 0\right]}^{i} \cap \mathbb{X}^{+}$. It vanishes on the boundary $\mathbb{R}_{<0}$ and $\frac{\delta}{2}$, and as $\delta \rightarrow 0$ the values on the boundary $w(1)$ of the discrete ball $B_{1}(0) \cap \mathcal{V}_{\left[\mathbb{C}_{\delta}, 0\right]}^{i}$ decays as $o(1)$. By the discrete Beurling estimate (see proof of Lemma 5.16) we can bound $\left|\sqrt{\frac{\pi}{2 \delta}}\left[L_{\delta}-R_{\delta}\right](z \delta)\right|$ for any $z \in \mathcal{V}_{\left[\mathbb{C}_{1}, 0\right]}^{i} \cap \mathbb{X}^{+}$from above by $C \delta^{1 / 2} o(1)$. Since by definition $\sqrt{\frac{\pi}{2 \delta}}\left[L_{\delta}-R_{\delta}\right](z \delta)=\sqrt{\frac{\pi \delta}{2}}\left[L_{1}-R_{1}\right](z)$, $\left[L_{1}-R_{1}\right](z)=0$, and thus $L_{\delta}=R_{\delta}$.

Then we conclude $k=\frac{1}{2}$ since $(1-k) \tilde{G}_{\left[\mathbb{C}_{\delta}, 0\right]}^{-}\left(e^{\pi i} \frac{\delta}{2}\right)=L_{\delta}\left(e^{\pi i} \frac{\delta}{2}\right)=i L_{\delta}\left(\frac{\delta}{2}\right)=$ $i k \tilde{G}_{\left[\mathbb{C}_{\delta}, 0\right]}^{+}\left(\frac{\delta}{2}\right)$ and $-\tilde{G}_{\left[\mathbb{C}_{\delta}, 0\right]}^{-}\left(e^{\pi i} \frac{\delta}{2}\right)=\tilde{G}_{\left[\mathbb{C}_{\delta}, 0\right]}^{+}\left(\frac{\delta}{2}\right)=G_{\left[\mathbb{C}_{\delta}, 0\right]}\left(\frac{3 \delta}{2}\right)$ again by the symmetry between real and imaginary parts of $G_{\left[\mathbb{C}_{\delta}, 0\right]}$.

\section{B. Contour Weights}

Here we prove the well-definedness of the spin-fermionic contour weights introduced in full generality in Sect. 4.1.

Recall from Sects. 3.0.2 and 4.1 the definition of $\gamma \in \mathcal{C}_{\Omega_{\delta}}^{\alpha_{1}, \ldots, \alpha_{2 n}}$ and the admissible choices of walks $\{\Gamma(\gamma)\}$ associated to it. Moreover recall the definition of the multipoint observable $F_{\left[\Omega_{\delta}, a\right]}$ from Definition 4.2. 
Proposition B.1. For any collection of distinct oriented medial vertices $\alpha_{1}, \ldots, \alpha_{2 n}$ and any $\gamma \in \mathcal{C}_{\Omega_{\delta}}^{\alpha_{1}, \ldots, \alpha_{2 n}}$, for every two admissible choices of walks, $\Gamma(\gamma), \Gamma^{\prime}(\gamma)$, we have

$$
(-1)^{\ell(\gamma \backslash \cup \Gamma(\gamma))} \prod_{\gamma^{\alpha_{j}, \alpha_{k} \in \Gamma(\gamma)}} \mathrm{s}_{\alpha_{j}, \alpha_{k}}\left(\gamma^{\alpha_{j}, \alpha_{k}}\right)=(-1)^{\ell\left(\gamma \backslash \cup \Gamma^{\prime}(\gamma)\right)} \prod_{\gamma^{\alpha_{j^{\prime}}, \alpha_{k^{\prime}} \in \Gamma(\gamma)}} \mathrm{s}_{\alpha_{j^{\prime}}, \alpha_{k^{\prime}}}\left(\gamma^{\alpha_{j^{\prime}}, \alpha_{k^{\prime}}}\right) .
$$

As a result, the function $F_{\left[\Omega_{\delta}, a\right]}$ is well-defined.

We will need the following two lemmas for the proof of the above proposition.

Lemma B.2. If $A, B$ are unions of disjoint loops in $\Omega_{\delta},(-1)^{\ell(A \oplus B)}=(-1)^{\ell(A)}(-1)^{\ell(B)}$.

Proof. For each of $A$ and $B$, fill in the faces of the lattice with spins, beginning with the plus boundary conditions, such that there is an edge between two faces if and only if they differ in sign. For each loop collection, we have the spin at zero $\sigma_{0}^{A}=(-1)^{\ell(A)}$ and $\sigma_{0}^{B}=(-1)^{\ell(B)}$.

The result follows after noting that $A \oplus B$ is identified with the spin configuration constructed by multiplying the spins of configurations $A$ and $B$ pointwise.

Lemma B.3. Suppose $l$ walks $w_{1}, \ldots, w_{l} \in\left\{\gamma_{1}, \ldots, \gamma_{n}, \gamma_{1}^{\prime}, \ldots, \gamma_{n}^{\prime}\right\}$ and $l$ distinct oriented medial vertices $\alpha^{1}, \ldots, \alpha^{l} \in\left\{\alpha_{1}, \ldots, \alpha_{2 n}\right\}$ form a cycle, i.e. $w_{1}$ connects (projections of) $\alpha^{1}$ with $\alpha^{2}$, w2 connects $\alpha^{2}$ with $\alpha^{3}, \ldots, w_{l}$ connects $\alpha^{l}$ with $\alpha^{l+1}:=\alpha^{1}$. Then we have,

$$
(-1)^{\ell\left(w_{1} \oplus \cdots \oplus w_{l}\right)}=s_{\alpha^{1}, \alpha^{2}}\left(w_{1}\right) \cdots s_{\alpha^{l}, \alpha^{1}}\left(w_{l}\right) .
$$

Proof. Fix a half-line $\Lambda=e^{i \theta} \mathbb{R}_{\geq 0}$ such that it is not parallel to any edges and is disjoint from all $\alpha^{1}, \ldots, \alpha^{l}$. Note that $\Omega \backslash \Lambda$ lifts to [ $\left.\Omega, 0\right]$ as two sheets. We now define two quantities: given a piecewise $C^{1}$ path in $\Omega \backslash\{0\}$, we can count the number of times $N_{\Lambda}$ that the path crosses $\Lambda$; given two points $a, z \in[\Omega, 0] \backslash \Lambda$, we define $S_{\Lambda}^{\alpha, \zeta}:=1$ if they belong to the same sheet in $[\Omega, 0]$ of $\Omega \backslash \Lambda$ and $S_{\Lambda}^{\alpha, \zeta}:=-1$ otherwise.

Concatenate the (possibly reversed) walks such that $w:=w_{1} \oplus w_{2} \oplus \cdots \oplus w_{l}$ is a continuous loop on $\Omega \backslash\{0\}$ starting from $\alpha^{1}$. Then clearly $\ell\left(w_{1} \oplus \cdots \oplus w_{l}\right) \equiv N_{\Lambda}(w)$ mod 2. Now it suffices to note that for any $j,(-1)^{N_{\Lambda}\left(w_{j}\right)} \mathrm{s}_{\alpha^{j}, \alpha^{j+1}}\left(w_{j}\right)=S_{\Lambda}^{\alpha^{j}, \alpha^{j+1}}$ so that

$$
\begin{aligned}
(-1)^{N_{\Lambda}(w)} \prod_{j=1}^{l} \mathrm{~s}_{\alpha^{j}, \alpha^{j+1}}\left(w_{j}\right) & =\prod_{j=1}^{l}(-1)^{N_{\Lambda}\left(w_{j}\right)} \mathrm{s}_{\alpha^{j}, \alpha^{j+1}}\left(w_{j}\right) \\
& =\prod_{j=1}^{l} S_{\Lambda}^{\alpha^{j}, \alpha^{j+1}}=S_{\Lambda}^{\alpha^{1}, \alpha^{1}}=1,
\end{aligned}
$$

from which the lemma follows.

Proof of Proposition B.1. First observe that $\gamma_{1}, \ldots, \gamma_{n}, \gamma_{1}^{\prime}, \ldots, \gamma_{n}^{\prime}$ and $a_{1}, \ldots, a_{2 n}$ are partitioned into disjoint cycles $P_{1}, \ldots, P_{l^{\prime}}$ in the sense of Lemma B.3 (suppose each $P_{j}$ is the resulting collection of loops of the form $w_{1} \oplus \cdots \oplus w_{l}$ ). Note that $P_{1} \oplus \cdots \oplus P_{l^{\prime}}=$ $\cup \Gamma(\gamma) \oplus \cup \Gamma^{\prime}(\gamma)$, and thus $(\gamma \backslash \cup \Gamma(\gamma)) \oplus\left(\gamma \backslash \cup \Gamma^{\prime}(\gamma)\right) \oplus P_{1} \oplus \cdots \oplus P_{l^{\prime}}=\emptyset$. By Lemma B.2,

$$
(-1)^{\ell(\gamma \backslash \cup \Gamma(\gamma))} \prod_{\gamma^{\alpha_{j}, \alpha_{k}} \in \Gamma(\gamma)} \mathrm{s}_{\alpha_{j}, \alpha_{k}}\left(\gamma^{\alpha_{j}, \alpha_{k}}\right) \cdot(-1)^{\ell\left(\gamma \backslash \cup \Gamma^{\prime}(\gamma)\right)} \prod_{\gamma^{\alpha_{j^{\prime}}, \alpha_{k^{\prime}} \in \Gamma(\gamma)}} \mathrm{s}_{\alpha_{j^{\prime}}, \alpha_{k^{\prime}}}\left(\gamma^{\alpha_{j^{\prime}}, \alpha_{k^{\prime}}}\right)
$$




$$
=(-1)^{\ell(\gamma \backslash \cup \Gamma(\gamma))}(-1)^{\ell\left(\gamma \backslash \cup \Gamma^{\prime}(\gamma)\right)} \prod_{j=1}^{l^{\prime}}(-1)^{\ell\left(P_{j}\right)}=(-1)^{\ell(\emptyset)}=1,
$$

concluding the proof.

\section{Explicit Pattern Probabilities}

In this section we give an example using Theorem 1.1 by computing explicitly the infinitevolume limit of and first-order conformal correction to a diagonal spin-spin correlation. On rotated lattices, $\Omega_{\delta} \subset \mathbb{C}_{\delta}$ with plus boundary, this corresponds to $\mathbb{E}_{\Omega_{\delta}}\left[\sigma_{0} \sigma_{2 \delta}\right]$ and is a quantity that appears in the study of the lattice level Ising stress tensor [BeHo18]. We then explain how the similar computation would be done for an " $\mathrm{L}$ " shaped spin-weighted correlation $\mathbb{E}_{\Omega_{\delta}}\left[\sigma_{0} \sigma_{(1+i) \delta} \sigma_{2 \delta}\right] / \mathbb{E}_{\Omega_{\delta}}\left[\sigma_{0}\right]$ and give the explicit values one gets from the explicit recursion process outlined to get values of the infinite-volume spin-fermion.

Corollary C.1. Consider the Ising model on $\Omega_{\delta}$ with plus boundary conditions and $0 \in \mathcal{F}_{\Omega_{\delta}}$; then, as $\delta \rightarrow 0$,

$$
\mathbb{E}_{\Omega_{\delta}}\left[\sigma_{0} \sigma_{2 \delta}\right]=\frac{2}{\pi}+\delta \cdot \frac{2}{\pi} \cdot r_{\Omega}^{-1}(0)+o(\delta) .
$$

Proof. We first observe that $\mathbb{E}_{\Omega_{\delta}}\left[\sigma_{0} \sigma_{2 \delta}\right]=\mathbb{E}_{\Omega_{\delta}}\left[\sigma_{0} \sigma_{(1+i) \delta} \sigma_{(1+i) \delta} \sigma_{2 \delta}\right]$ and

$$
\mathbb{E}_{\Omega_{\delta}}\left[\epsilon\left(a_{1}\right) \epsilon\left(a_{2}\right)\right]=\frac{1}{2}+\mathbb{E}_{\Omega_{\delta}}\left[\sigma_{0} \sigma_{(1+i) \delta} \sigma_{(1+i) \delta} \sigma_{2 \delta}\right]-\frac{\sqrt{2}}{2}\left(\mathbb{E}_{\Omega_{\delta}}\left[\sigma_{0} \sigma_{(1+i) \delta}\right]+\mathbb{E}_{\Omega_{\delta}}\left[\sigma_{(1+i) \delta} \sigma_{2 \delta}\right]\right),
$$

where $a_{1}=\frac{1+i}{2}, a_{2}=\frac{3+i}{2}$. By the first-order Taylor expansion of [HoSm13] (after rescaling) for the energy density $\left(\mathbb{E}_{\Omega_{\delta}}\left[\epsilon\left(a_{1}\right)\right]=-\delta \cdot \frac{\sqrt{2}}{\pi} \cdot r_{\Omega}^{-1}(0)+o(\delta)\right)$, this implies that as $\delta \rightarrow 0$,

$$
\mathbb{E}_{\Omega_{\delta}}\left[\sigma_{0} \sigma_{2 \delta}\right]=\frac{1}{2}+\mathbb{E}_{\Omega_{\delta}}\left[\epsilon\left(a_{1}\right) \epsilon\left(a_{2}\right)\right]+\delta \cdot \frac{2}{\pi} \cdot r_{\Omega}^{-1}(0)+o(\delta) .
$$

From this it suffices to compute the first-order asymptotics of $\lim _{\delta \rightarrow 0} \mathbb{E}_{\Omega_{\delta}}\left[\epsilon\left(a_{1}\right) \epsilon\left(a_{2}\right)\right]$. In order to do so, we consider the antisymmetric matrix

$$
\mathbf{F}^{\left\{e_{k}\right\}}=\left(\begin{array}{cccc}
0 F_{\mathbb{C}_{1}}^{a_{1}^{+} a_{2}^{+}} & F_{\mathbb{C}_{1}}^{a_{1}^{+} a_{2}^{-}} & F_{\mathbb{C}_{1}}^{\dagger a_{1}^{+} a_{1}^{-}} \\
0 & F_{\mathbb{C}_{1}}^{\dagger a_{2}^{+} a_{2}^{-}} & F_{\mathbb{C}_{1}}^{a_{2}^{+} a_{1}^{-}} \\
& 0 & F_{\mathbb{C}_{1}}^{a_{2}^{-} a_{1}^{-}} \\
& & 0
\end{array}\right)=\left(\begin{array}{rrrr}
0 & a & b & c \\
0 & d & e \\
0 & 0 & f \\
& & 0
\end{array}\right)
$$

where we choose orientations $o_{1}^{+}=e^{7 \pi i / 4}$ and $o_{2}^{+}=e^{5 \pi i / 4}$ on $\mathbb{C}_{1}$. Plugging in the explicit values from the observable defined in Eq. (3.1) and using the definition $F_{\mathbb{C}_{1}}^{\alpha, \zeta}=$ $\operatorname{Re}\left[i \sqrt{p} H_{\mathbb{C}_{1}}^{\alpha, z}\right]$, where we choose the principal branch of the square root, observe that

$$
\begin{aligned}
a=-\frac{1}{2 \pi}+\frac{\sqrt{2}}{2 \pi}+\frac{1}{4}, & b=-\frac{1}{4}+\frac{1}{2 \pi}, \\
c=0, \quad d & =0,
\end{aligned}
$$




$$
e=-\frac{1}{4}+\frac{1}{2 \pi}, \quad f=\frac{1}{2 \pi}+\frac{\sqrt{2}}{2 \pi}-\frac{1}{4} .
$$

Combined with $\operatorname{Pf}\left(\mathbf{F}^{\left\{e_{k}\right\}}\right)=a f-b e+c d$ and $\lim _{\delta \rightarrow 0} \mathbb{E}_{\Omega_{\delta}}\left[\sigma_{0} \sigma_{2 \delta}\right]=\frac{1}{2}+4 \operatorname{Pf}\left(\mathbf{F}^{\left\{e_{k}\right\}}\right)$ (where we applied Theorem 1.1 to $\mathbb{E}_{\Omega_{\delta}}\left[\epsilon\left(a_{1}\right) \epsilon\left(a_{2}\right)\right]$ ),

$$
\lim _{\delta \rightarrow 0} \mathbb{E}_{\Omega_{\delta}}\left[\sigma_{0} \sigma_{2 \delta}\right]=\mathbb{E}_{\mathbb{C}_{1}}\left[\sigma_{0} \sigma_{2 \delta}\right]=\frac{2}{\pi} .
$$

In order to compute the constant in the conformal correction, note that the matrix $\mathbf{E}^{\left\{e_{k}\right\}}$ is given by $E_{\mathbb{C}_{1}}^{\alpha, \zeta}=\operatorname{Re}\left[\frac{i \sqrt{p} \overline{\sqrt{o}}}{\sqrt{2} \pi}\right]$ so that, here,

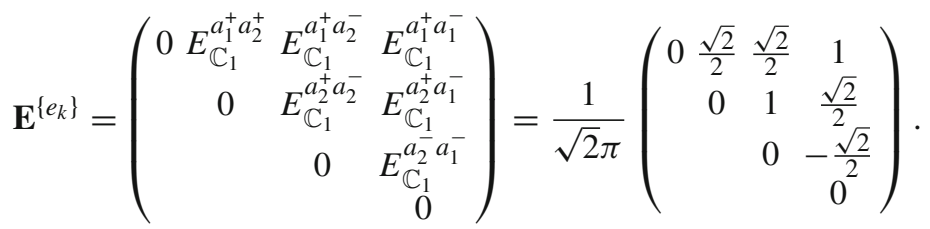

Inverting $\mathbf{F}^{\left\{e_{k}\right\}}$ by hand, and using the Pfaffian expansion formula $\operatorname{Pf}(A+\delta B)=$ $\operatorname{Pf}(A)+\delta \operatorname{Pf}(A) \operatorname{Tr}\left(A^{-1} B\right)$, along with the above expressions, we see that, in fact, $4 D_{\mathbf{E}^{\left\{e_{k}\right\}}} \operatorname{Pf}\left(\mathbf{F}^{\left\{e_{k}\right\}}\right)=4 \operatorname{Pf}\left(\mathbf{F}^{\left\{e_{k}\right\}}\right) \operatorname{Tr}\left(\left(\mathbf{F}^{\left\{e_{k}\right\}}\right)^{-1} \mathbf{E}^{\left\{e_{k}\right\}}\right)=0$ which, combined with Theorem 1.1 implies the desired geometric correction.

Corollary C.2. Consider the Ising model on $\Omega_{\delta}$ with plus boundary conditions and $0 \in \mathcal{F}_{\Omega_{\delta}}$; then as $\delta \rightarrow 0$,

$$
\frac{\mathbb{E}_{\Omega_{\delta}}\left[\sigma_{0} \sigma_{(1+i) \delta} \sigma_{2 \delta}\right]}{\mathbb{E}_{\Omega_{\delta}}\left[\sigma_{0}\right]}=2(\sqrt{2}-1)+\delta \cdot \frac{5 \sqrt{2}-7}{2} \cdot \operatorname{Re}\left[\left.\partial_{z} \log r_{\Omega}(z)\right|_{z=0}\right]+o(\delta) .
$$

Proof. First observe that the edge $e$ separating $\sigma_{(1+i) \delta}$ from $\sigma_{2 \delta}$ has midpoint at $\delta a$ where $a=\frac{3}{2}+\frac{i}{2}$.

$$
\frac{\mathbb{E}_{\Omega_{\delta}}\left[\sigma_{0} \epsilon_{[0]}(\delta a)\right]}{\mathbb{E}_{\Omega_{\delta}}\left[\sigma_{0}\right]}=\frac{\mathbb{E}_{\Omega_{\delta}}\left[\sigma_{0} \sigma_{(1+i) \delta} \sigma_{2 \delta}\right]}{\mathbb{E}_{\Omega_{\delta}}\left[\sigma_{0}\right]}-\mu_{a}
$$

They by Eq. (6.1), picking an s-orientation $o$ on $a$,

$$
\mu_{a}=\sqrt{o}\left[H_{\left[\mathbb{C}_{1}, 0\right]}^{a^{o}}\left(a_{+}^{o}\right)+H_{\left[\mathbb{C}_{1}, 0\right]}^{a^{o}}\left(a_{-}^{o}\right)\right]
$$

To compute the front and back values of $H_{\left[\mathbb{C}_{1}, 0\right]}^{a^{o}}\left(a_{ \pm}\right)$we have implemented the recursion procedure for $H$ using Mathematica as outlined in Proposition 3.22 and Corollary 3.24 (see also Fig. 5). That yields that

$$
\mu_{3 / 2+i / 2}=2 \sqrt{2}-2 .
$$

Now by Theorem 1.2, we wish to compute $\mathcal{P}_{[0]}^{a}=(-2) \operatorname{Pf}\left(\mathbf{F}_{[0]}^{a}\right)$ but since $\mathbf{F}_{[0]}^{a}$ is a $2 n \times 2 n$ antisymmetric matrix that is zero on its anti-diagonal, $\mathcal{P}_{[0]}^{a}=0$. On the other hand, that implies that $Q_{[0]}^{a}=\mathbf{E}_{[0]}^{a}$ whose entries are given by Definition 6.4 in terms of values of $G_{\left[\mathbb{C}_{1}, 0\right]}$ and $\tilde{G}_{\left[\mathbb{C}_{1}, 0\right]}^{ \pm}$evaluated on oppositely oriented $a^{o^{ \pm}}$. Via the explicit construction 
of the slit-plane harmonic measure recursion procedure outlined in Remark 3.27, one can calculate

$$
E_{\left[\mathbb{C}_{1}, 0\right]}^{a^{o^{+}}, a^{o^{-}}}=5 \sqrt{2}-7
$$

Putting these together and plugging in to the expansion given by Theorem 1.2 for the spin-weighted correlation, we obtain the desired geometric correction.

\section{References}

[Bax89] Baxter, R. Exactly Solved Models in Statistical Mechanics Harcourt Brace Jovanovich Publishers, Academic Press Inc. London 1989

[BPZ84] Belavin, A.A., Polyakov, A.M., Zamolodchikov, A.B.: Infinite conformal symmetry in twodimensional quantum field theory. Nucl. Phys. B 241(2), 333-380 (1984)

[Bo07] Boutillier, C.: Pattern densities in non-frozen dimer models. Commun. Math. Phys. 271(1), 5591 (2007).

[BeHo16] Benoist, S., Hongler, C.: The scaling limit of critical Ising interfaces is CLE(3) (to appear in Ann. Probab.) (2016)

[BeHo18] Benoist, S., Hongler, C.: in preparation

[BoDT09] Boutillier, C., de Tilière, B.: The critical Z-invariant Ising model via dimers: locality property. Commun. Math. Phys. 301(2), 473-516 (2011)

[BoDT08] Boutillier, C., de Tilière, B.: The critical Z-invariant Ising model via dimers: the periodic case. Prob. Theory Relat. Fields 147(3), 379-413 (2010)

[BuGu93] Burkhardt, T., Guim, I.: Conformal theory of the two-dimensional Ising model with homogeneous boundary conditions and with disordered boundary fields. Phys. Rev. B (1) 47, 14306$14311(1993)$

[Car84] Cardy, J.: Conformal invariance and surface critical behavior. Nucl. Phys. B 240, 514532 (1984)

[CDHKS14] Chelkak, D., Duminil-Copin, H., Hongler, C., Kemppainen, A., Smirnov, S.: Convergence of Ising Interfaces to Schramm's SLE curves. C. R. Math. Acad. Sci. Paris 352(2), 157-161 (2014)

[CCK17] Chelkak, D., Cimasoni, D., Kassel, A.: Revisiting the combinatorics of the 2D Ising model. Annales de l'Institut Henri Poincaré (D) 4(3), 309-385 (2017)

[CGS17] Chelkak, D., Glazman, A., Smirnov, S.: Discrete stress-energy tensor in the loop O(n) model (2017). arXiv:1604.06339

[ChHo18] Chelkak, D., Hongler, C.: in preparation

[CHI15] Chelkak, D., Hongler, C., Izyurov, K.: Conformal invariance of spin correlations in the planar Ising model. Ann. Math. 181(3), 1087-1138 (2015)

[ChIz13] Chelkak, D., Izyurov, K.: Holomorphic spinor observables in the critical Ising model. Commun. Math. Phys. 322(2), 302-303 (2013)

[ChSm11] Chelkak, D., Smirnov, S.: Discrete complex analysis on isoradial graphs. Adv. Math. 228, 15901630 (2011)

[ChSm12] Chelkak, D., Smirnov, S.: Universality in the 2D Ising model and conformal invariance of fermionic observables. Invent. Math. 189(3), 515-580 (2012)

[CFL28] Courant R., Friedrichs K., Lewy, H.: Uber die partiellen Differenzengleichungen der mathematischen. Phys. Math. Ann. 100, 32-74 (1928)

[DMS97] Di Francesco, P., Mathieu, P., Sénéchal, D.: Conformal Field Theory, Graduate Texts in Contemporary Physics. Springer, New York (1997)

[DHN11] Duminil-Copin, H., Hongler, C., Nolin, P.: Connection probabilities and RSW-type bounds for the FK Ising model. Commun. Pure Appl. Math. 64(9), 1165-1198 (2011)

[Dub15] Dubédat, J.: Dimers and families of Cauchy-Riemann operators I. J. Am. Math. Soc. 28, 10631167 (2015)

[Gri06] Grimmett, G.: The Random-Cluster Model. Volume 333 of Grundlehren der Mathematischen Wissenschaften. Springer, Berlin (2006)

[Hon10] Hongler, C.: Conformal Invariance of Ising model Correlations. Ph.D. thesis (2010)

[HoKy10] Hongler, C., Kytölä, K.: Ising Interfaces and Free Boundary Conditions. J. Am. Math. Soc. 26(4), 1107-1189 (2013). https://doi.org/10.1090/S0894-0347-2013-00774-2

[HKV17] Hongler, C., Kytölä, K., Viklund, F.: Conformal Field Theory at the Lattice Level: Discrete Complex Analysis and Virasoro Structure, arXiv:1307.4104 (2017)

[HoSm13] Hongler, C., Smirnov, S.: The energy density in the critical planar Ising model. Acta Math. 211(2), 191-225 (2013) 
[Isi25] Ising, E.: Beitrag zur Theorie des Ferromagnetismus. Z. Phys. 31, 253-258 (1925)

[KaCe71] Kadanoff, L., Ceva, H.: Determination of an operator algebra for the two-dimensional Ising model. Phys. Rev. B (3) 3, 3918-3939 (1971)

[Kau49] Kaufman, B.: Crystal statistics. II. Partition function evaluated by spinor analysis. Phys. Rev., II. Ser. 76, 1232-1243 (1949)

[KaOn49] Kaufman, B., Onsager, L.: Crystal statistics. III. Short-range order in a binary Ising lattice. Phys. Rev. II. Ser. 76, 1244-1252 (1949)

[KaOn50] Kaufman, B., Onsager, L.: Crystal statistics. IV. Long-range order in a binary crystal. Unpublished typescript (1950)

[Ken00] Kenyon, R.: Conformal invariance of domino tiling. Ann. Probab. 28, 759-795 (2002)

[Kes87] Kesten, H.: Hitting probabilities of random walks on $\mathbb{Z}^{d}$. Stoch. Processes Appl. 25, 165184 (1987)

[KrWa41] Kramers, H.A., Wannnier, G.H.: Statistics of the two-dimensional ferromagnet. I.. Phys. Rev. (2) 60, 252-262 (1941)

[LaLi04] Lawler, G.F., Limic, V.: The Beurling estimate for a class of random walks. Electron. J. Probab. 9, 846-861 (2004)

[Lel55] Lelong-Ferrand, J.: Représentation conforme et transformations à intègrale de Dirichlet bornée. Gauthier-Villars, Paris (1955)

[Len20] Lenz, W.: Beitrag zum Verständnis der magnetischen Eigenschaften in festen Körpern. Phys. Z. 21, 613-615 (1920)

[LuS112] Lubetzky, E., Sly, A.: Critical Ising on the square lattice mixes in polynomial time. Commun. Math. Phys. 313, 815-836 (2012)

[Mer01] Mercat, C.: Discrete Riemann surfaces and the Ising model. Commun. Math. Phys. 218, 177216 (2001)

[McWu73] McCoy, B.M., Wu, T.T.: The Two-Dimensional Ising Model. Harvard University Press, Cambridge, MA (1973)

[Ons44] Onsager, L.: Crystal statistics. I. A two-dimensional model with an order-disorder transition. Phys. Rev. (2) 65, 117-149 (1944)

[Pal07] Palmer, J.: Planar Ising correlations. Birkhäuser, Basel (2007)

[SMJ77] Sato, M., Miwa, T., Jimbo, M.: Studies on holonomic quantum fields, I-IV. Proc. Jpn Acad. Ser. A Math. Sci. 53(1), 6-10; 53(1), 147-152; 53(1), 153-158; 53(1), 183-185 (1977)

[Smi06] Smirnov, S.: Towards conformal invariance of 2D lattice models. In: Sanz-Solé, M. et al.(ed.) Proceedings of the International congress of Mathematicians (ICM), Madrid, Spain, August 2230, 2006. Volume II: Invited lectures, pp. 1421-1451. Zürich: European Mathematical Society (EMS) (2006)

[Smi10] Smirnov, S.: Conformal invariance in random cluster models. I. Holomorphic fermions in the Ising model. Ann. Math. 172(2), 1435-1467 (2007)

[Yan52] Yang, C.N.: The spontaneous magnetization of a two-dimensional Ising model. Phys. Rev. (2) 85, 808-816 (1952)

Communicated by H. Duminil-Copin 